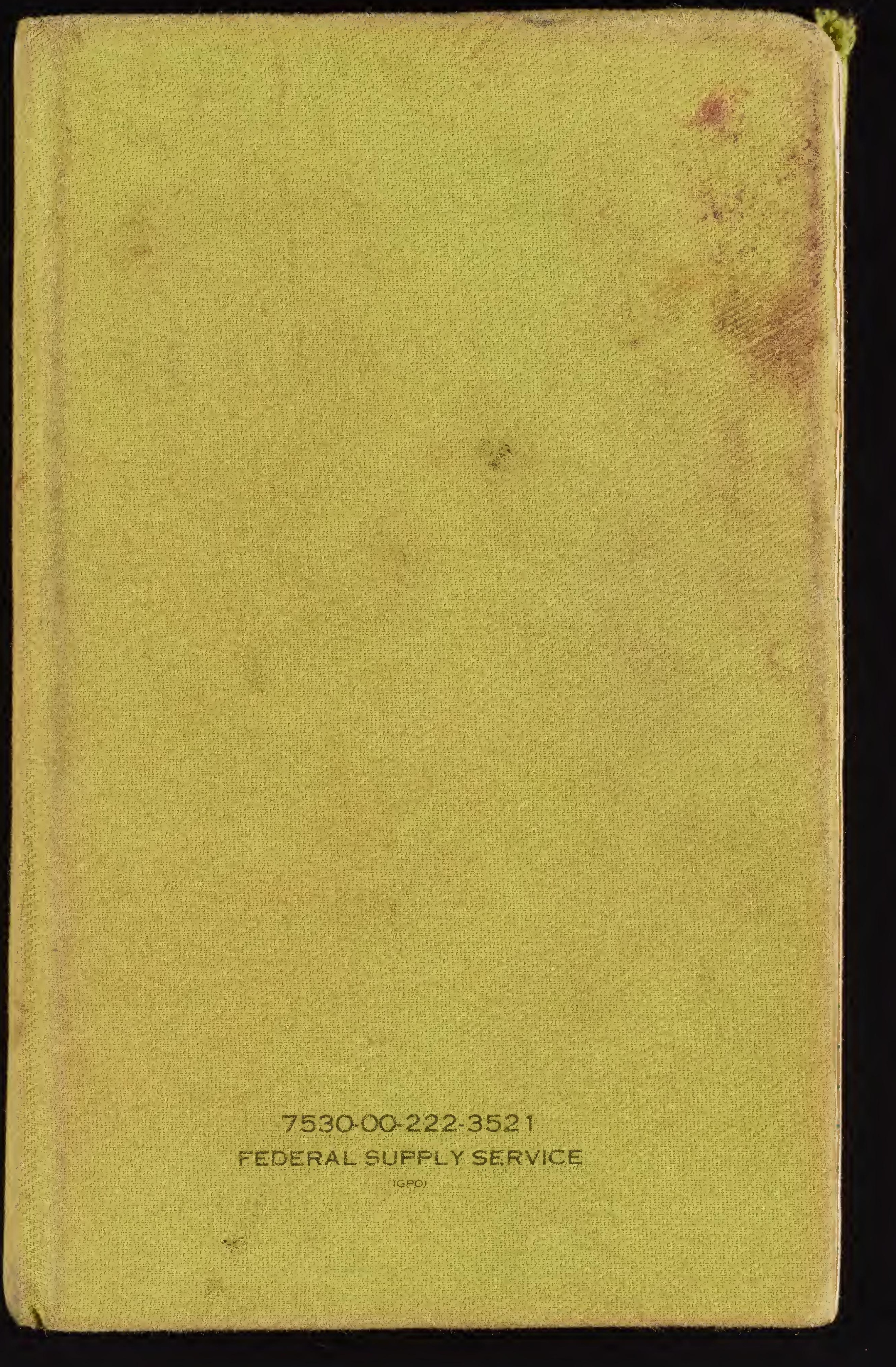





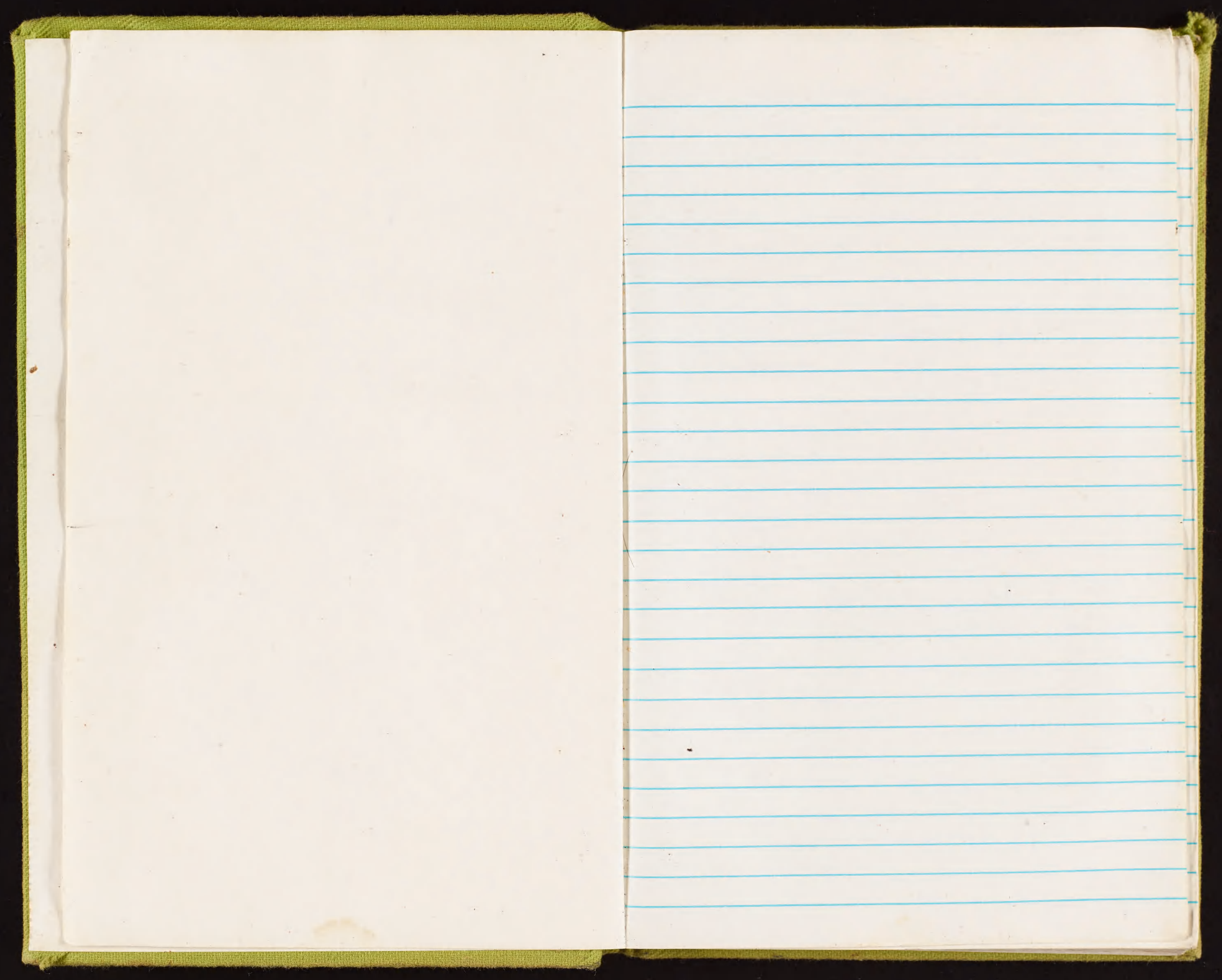


feb 9 - brozed Aca)ul

- oucrest $R$ caln

Reurse order

20

Busel Aabdach

T. $k_{1, j}, \mathrm{i}$. 6.is start

Penc $4 / 6 m$

Vine flower probe

ol.-e sprou

$$
\text { [Lat the.te) }
$$

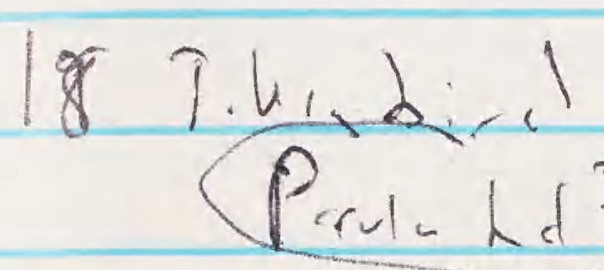

$$
\begin{array}{r}
\text { M.a S. vircoling } \\
2 \text { T.Moukgbirls }
\end{array}
$$

17.4 \& $17 \mathrm{~m} q$ 4ellow wibler in $/ 5 \mathrm{~m}$ ing $2 \times 4$ " nifren
$20 \mathrm{~m}$

ll. 8 27. nucyid

t. k, birl

w. e. seedecter

II.T T. Pawee

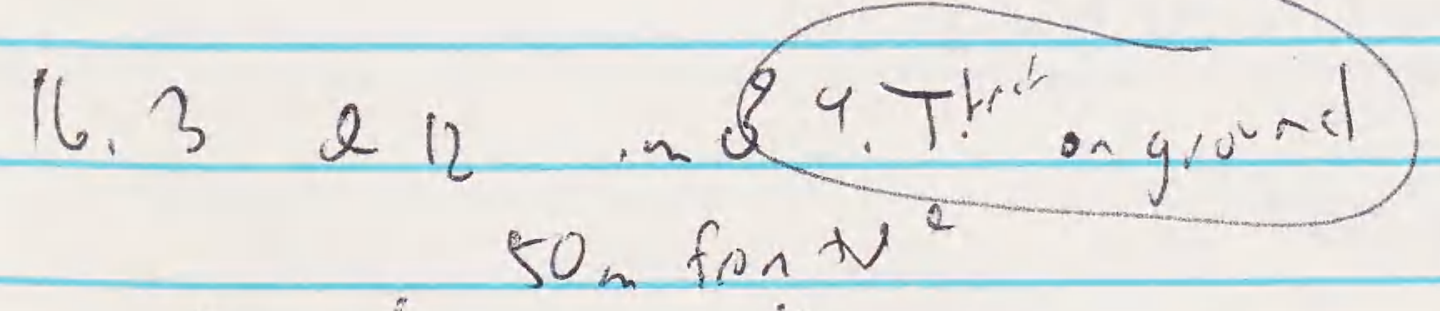

$\checkmark$ olusperen

dite spen 


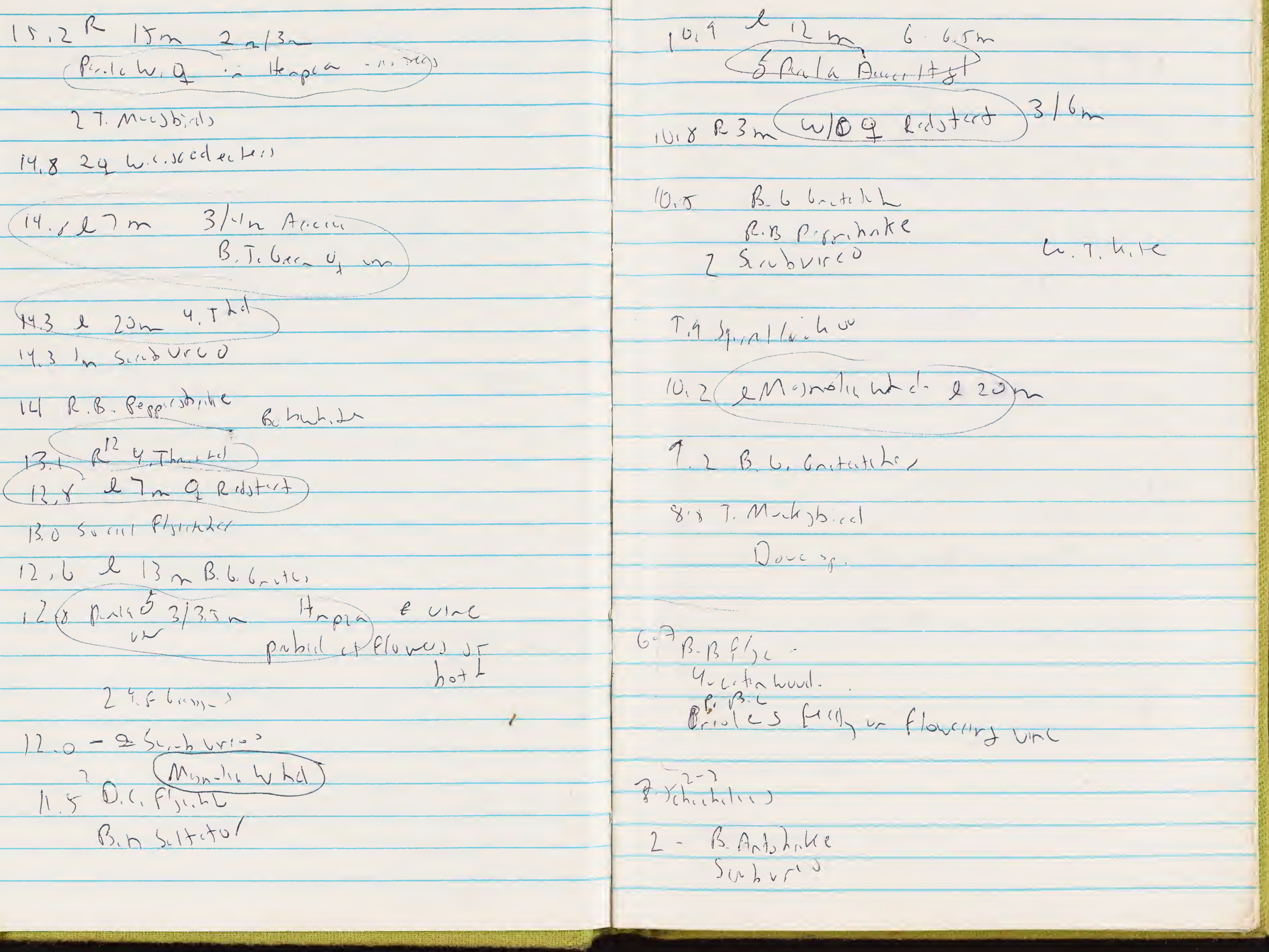


1.90 .3 renow

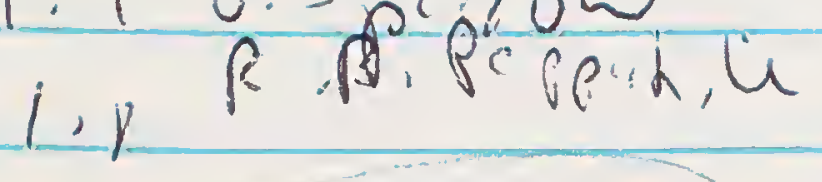

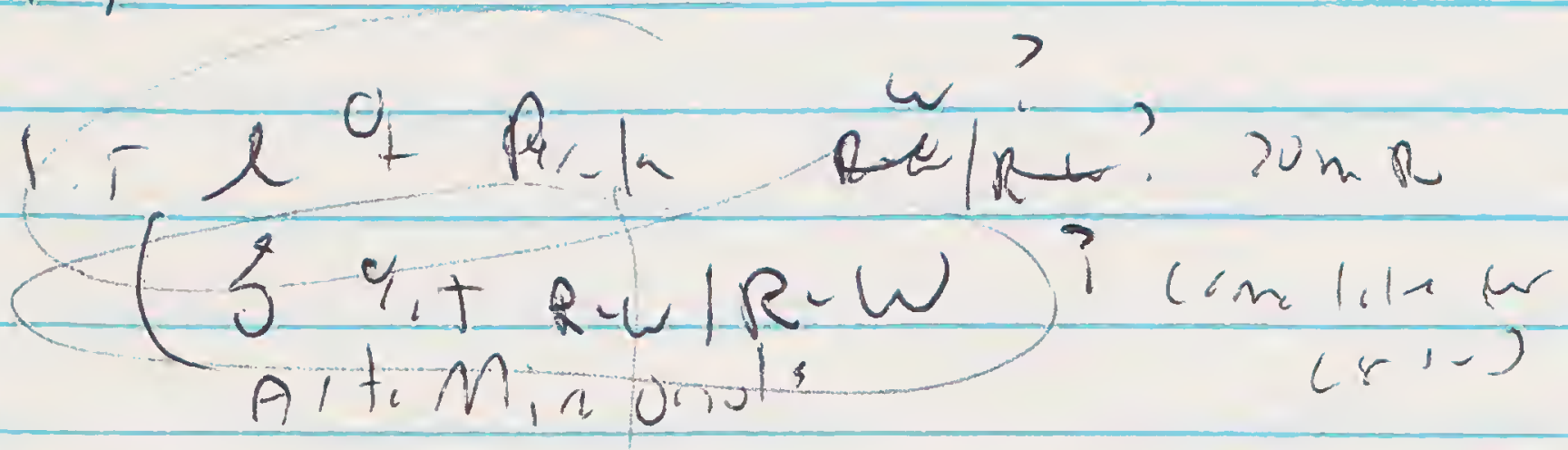

$$
\text { hemilg serit? }
$$

1.2 ad $\&$ Marlink (spurg?)

$$
0.5 \mu+R
$$

2 T.t hids

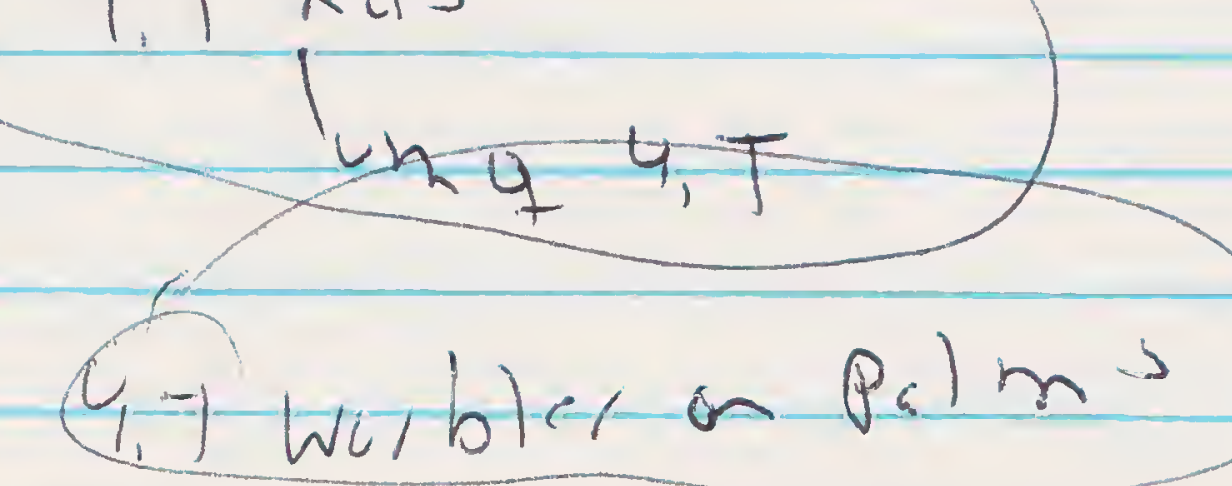

B.h. Sistitu'

O. Sorsow p广

11

PR A.Mire onok - Ore culvbred of

$+c$

B.C.0.101e

Pr 12 un lear flyc.

Gried Ac Ined 8:80urent

$40 \mathrm{~m}$

P l.S.t

50

ing

150

505

行

100

so

$\underset{20}{100}$

$100 \quad L+3 m a n$

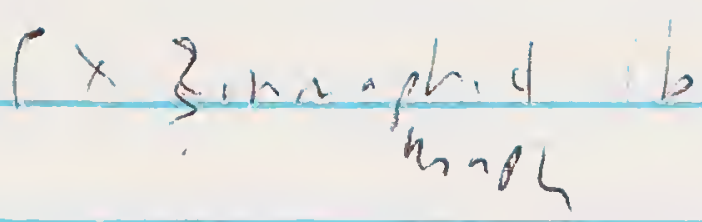

100

$1003 m_{n}$ lochers catat is 


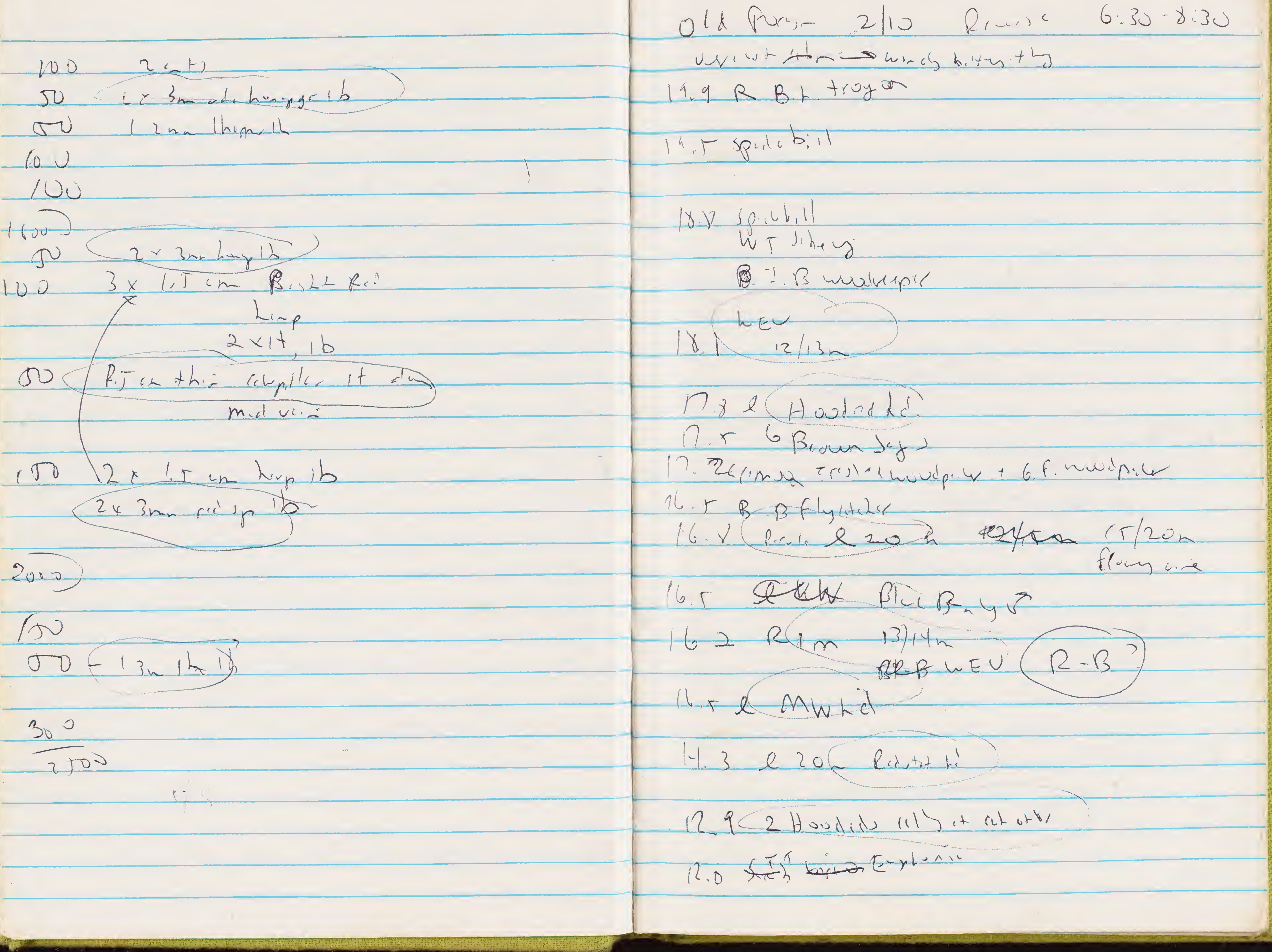


11.2R Ridutid di.

spidcbill

$10 \cdot 2$ 0. vadmépe

$4-10 \quad 6-7$ B. J)

$2 l, B$ TuL

Ancer.

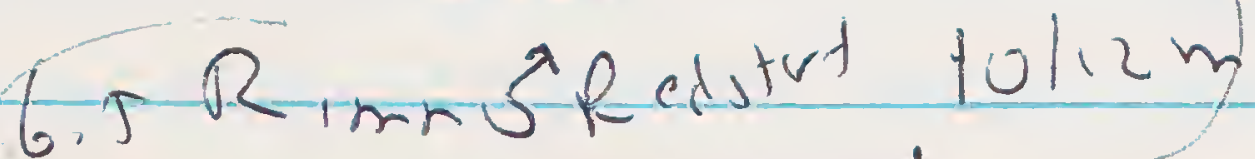

7 Carolinwion

$\cos 150$

$15125 m+14$

5. 6 p. 4 b. ll

5,4 ouk Liogel $x$

J,3 Soroks Brin houlgiv

$$
\text { youtitin Plye, }
$$

thtore ath $2-x$ bix

Qf. 2 R WIEVUKo chatter

$s_{p+i} h, \|$

4.3 fintat chip

$$
\begin{aligned}
& \text { 2.5 e Y.T, U1,10Lal }
\end{aligned}
$$

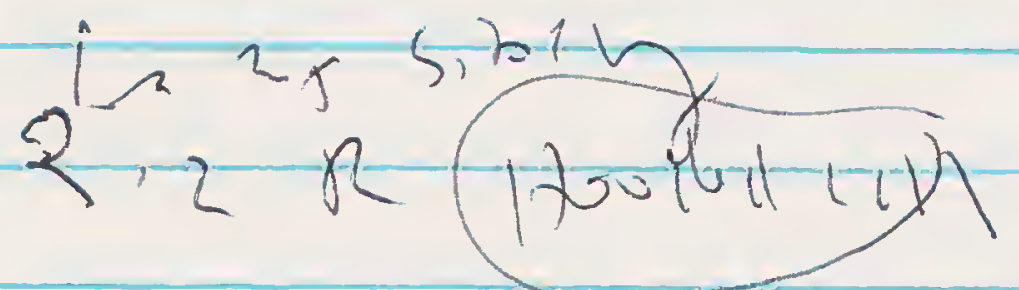

Inaets 1 I Tm

12 elest

100 3nrspice ib

rou 4hespivib.

100 C.m lisitith h

103

100 umr spur ih

200

200

200

180

13.

200

100

10.0

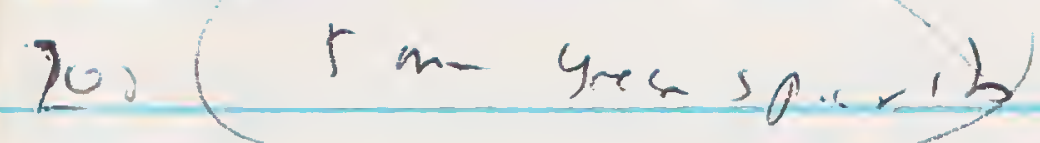

1005 a a d or b.r., l $+1 t$

$2 \Omega(2 m-4 \cdots, 1 b$

ito

23.0

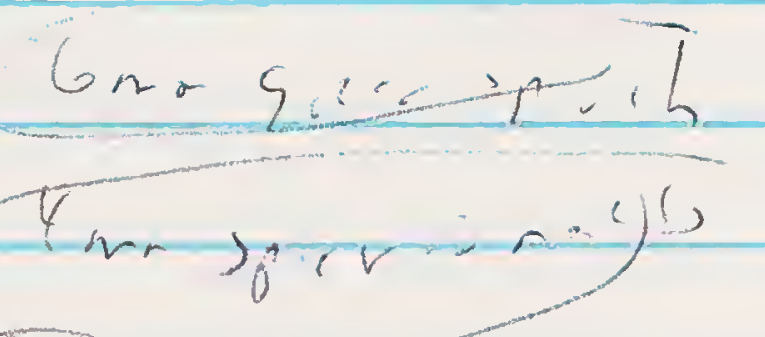

$100 \quad a_{m n}^{5} i^{2} b$

100

$2 / 8$ 


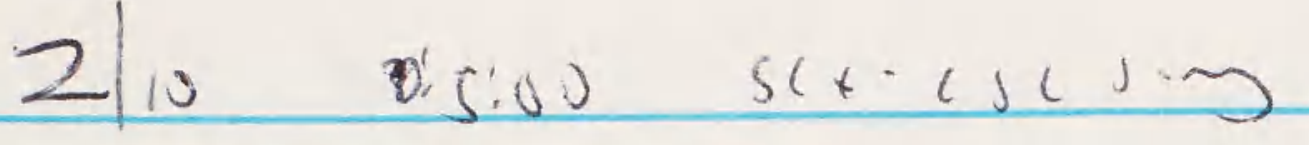

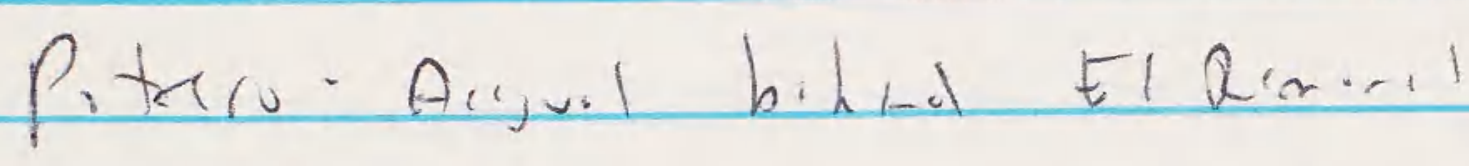

PotequT

$\sin 3$ पT

Acyullidice - q Redotert

frombiec $x$ - q Redutact

Aisual of Ridotet
L/n sectesty

New 10, , , the 1 ln $11-12$

$\operatorname{inn} v^{2} R$ id

skm

$q_{P} B+L$

q Mosnolic

of Baw wrbler

2 incut be

$3 c+2$

W.c.effthid:

2Raw

Murhinith

IN,

ठ) Ma 66

o B $+w$

6BTber in Alices unor

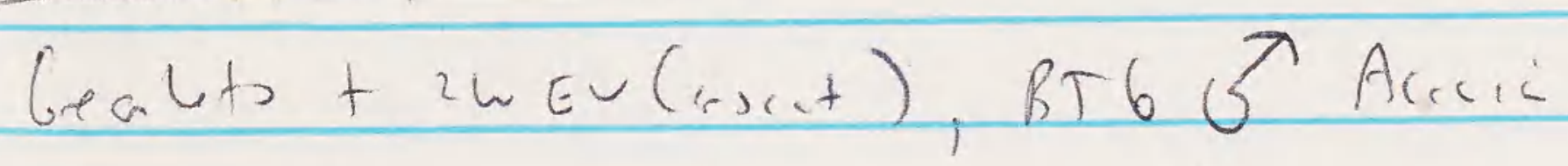

$\rightarrow$ Buw wobles $8 \times$ hix (i. dixe pube ih, de

1 ka..e deal lat oft live k.t qMW ad o ked trt 
3 How all

q $n w_{3}$

Red erad.

Rad od

BT6 12/18n Acur Ibjtan

$$
\text { Te. } 12
$$

2d tilirote $\quad u_{n}=6$ old Asul anW s-7yars?

VEV ir w

wtev

sinbure

Colith d-sents

$6 r a-4 \cdot \operatorname{low} c h e t$

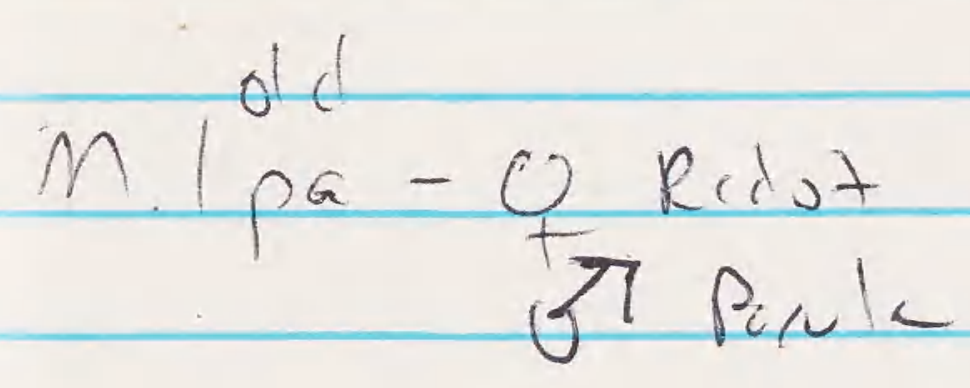

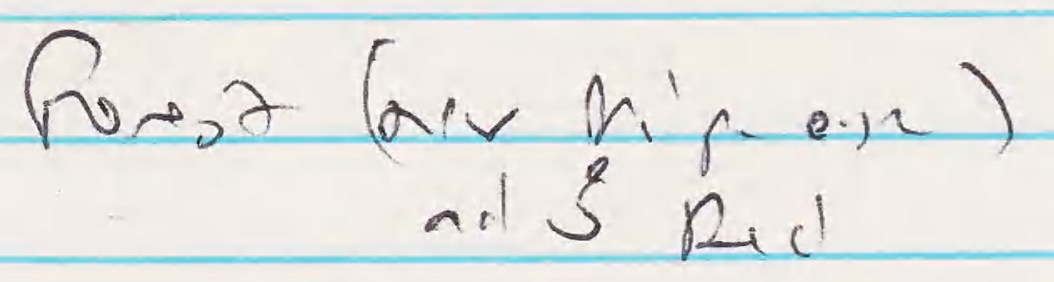

fors od 3 R. . 
2

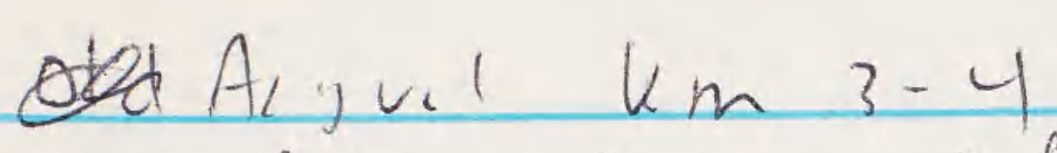

de forest Treased set nels

lota of Irme in frow

o Ristert

ouinbiri

o mw

Rema in tres

Sin 2 (itbid),

$$
+3 \cdot B f^{\prime}(x)
$$

ha loto of utbial 11)

q Mu Aiguilese lgrlpink at fork = roud

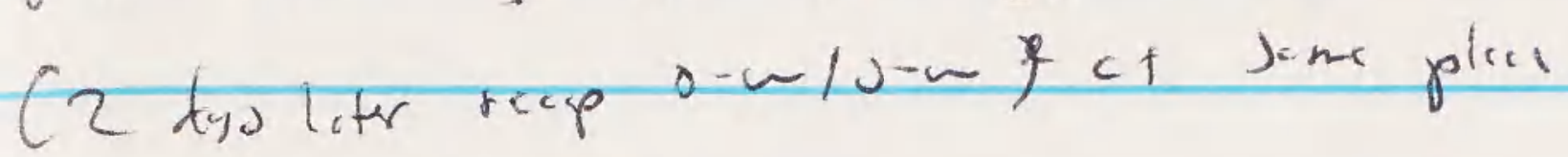

$2 / 13$

renncts $\rightarrow$ iridolid ford

PT 11

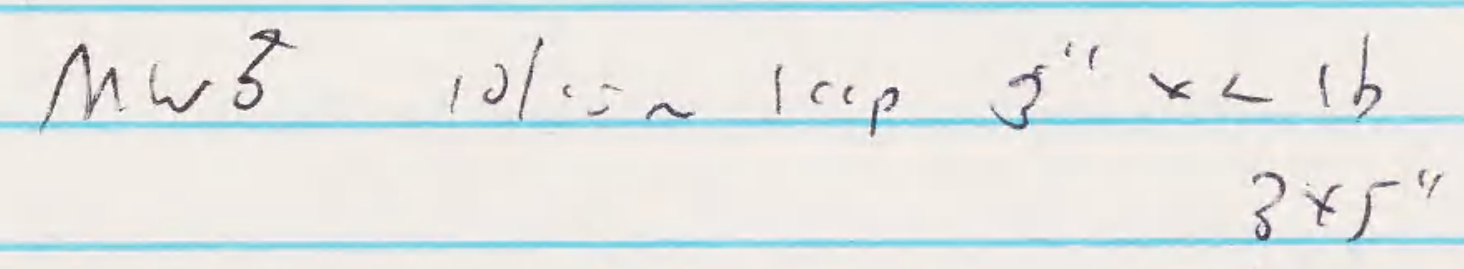

in fre.tirstre-nofergivary

-11 nist nets an old fores rosited 2 doobds one at $17,18.5$ Rod pres
Plf?
Plf? 
No brath

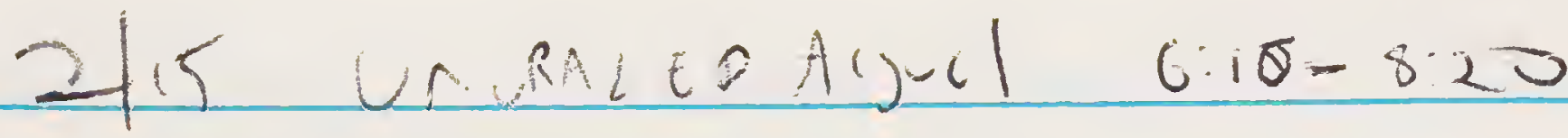

6.15 cleast colm

0.6 Arsuling

6.7R S. vire

$0>20>2+$

1.2 e buinu

1.2 R B. H r int

l.y $\ell$ B. $C$ in.ludL

2,2 e S.B.w.th

$$
\text { Res } 240+h a+41)
$$$$
3, r \text { Siaburio }
$$

3ir ezs Burist

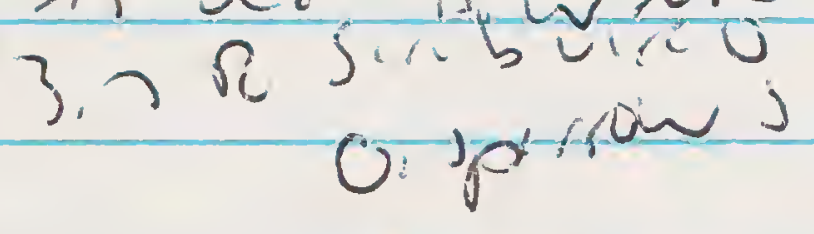

$$
\text { foridrus }
$$

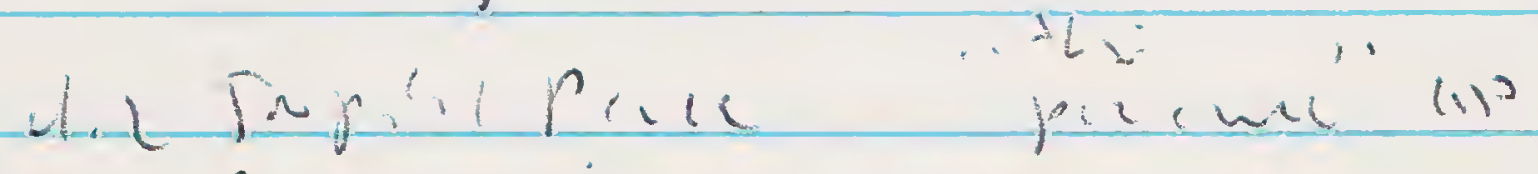

$4,<p \cdot p p r i t$ i

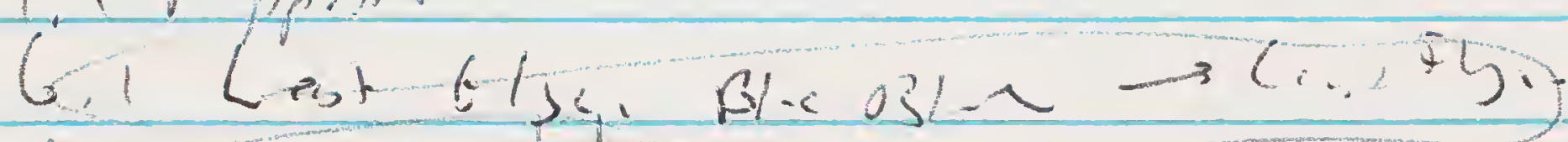

G. ¿R Huon h

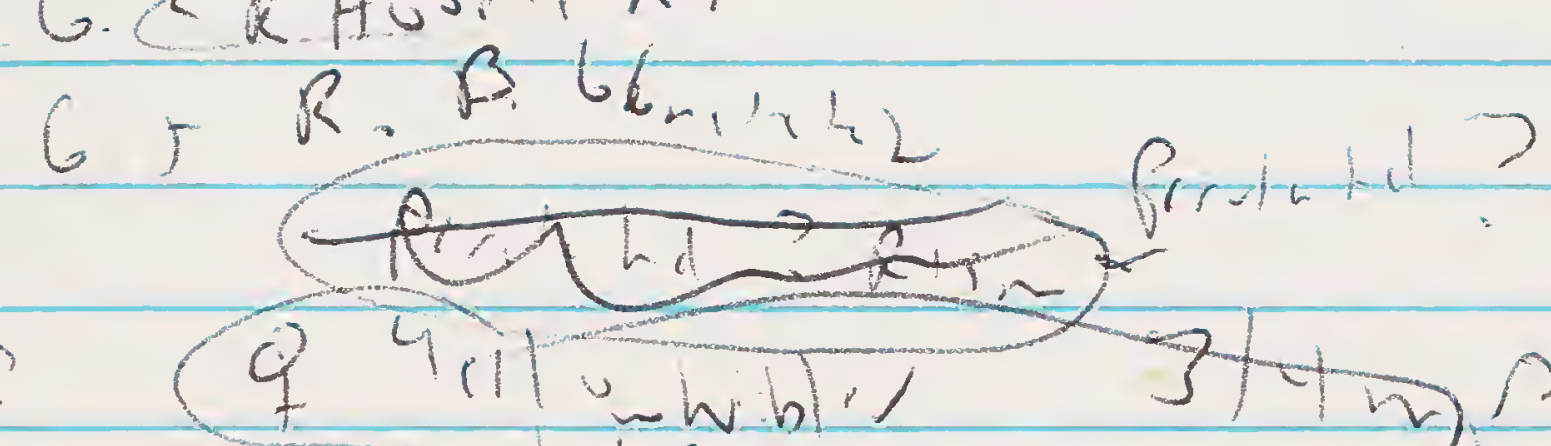

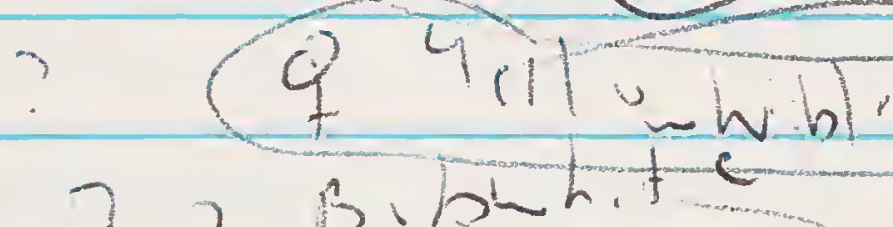

7.2 B.bunte
2. e Howld?

$y=B$. H S ItW
9 t T.Mnshid

9.\}RS.lisio

ip 90 sine

T. 8 R2Om Lasthe wh

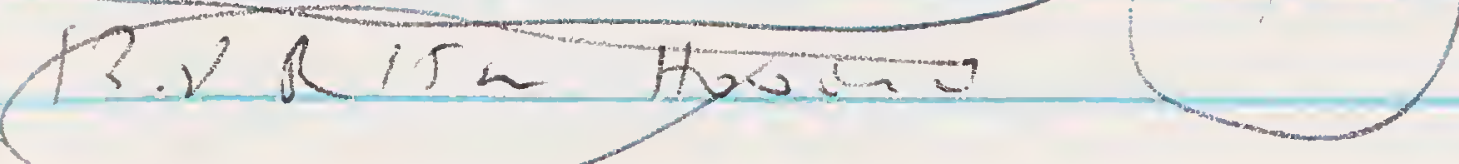

IS? YBEW. :

$15.2 \times B 6 b+i t h$

H. 22 lerint?

16.6 rever

16.15 lo pilums

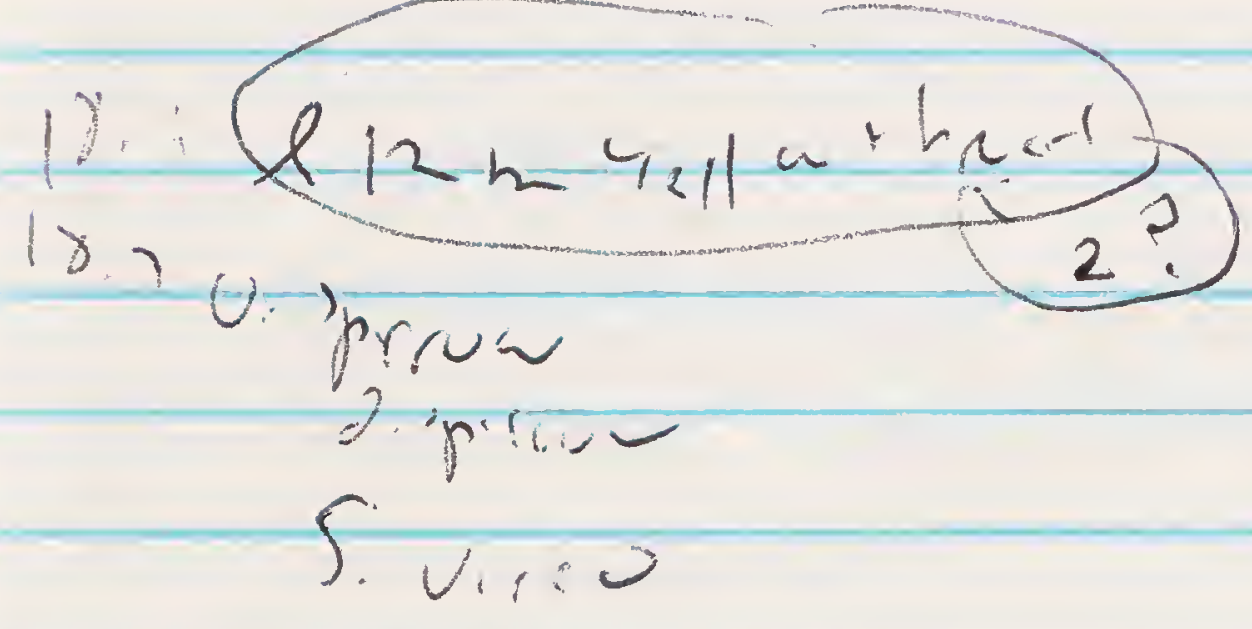


Bjorahd has

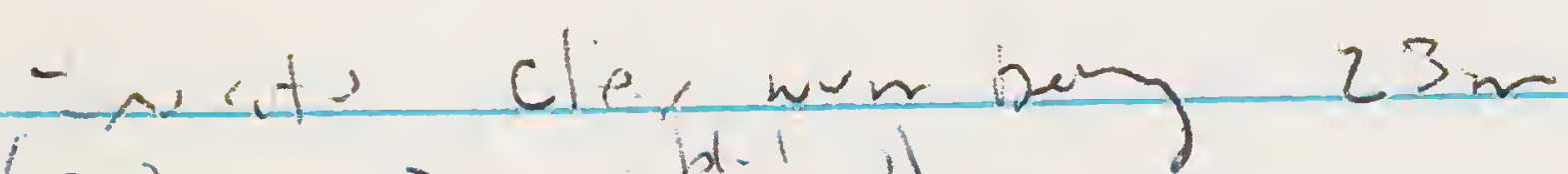

(6) 20 2abith ib

Inerisubur $14 ?$

$$
\begin{aligned}
& \text { Jna , him a.j It } \\
& 600 \leq 3 \times 4 \text { has solir }\} \text { th }
\end{aligned}
$$

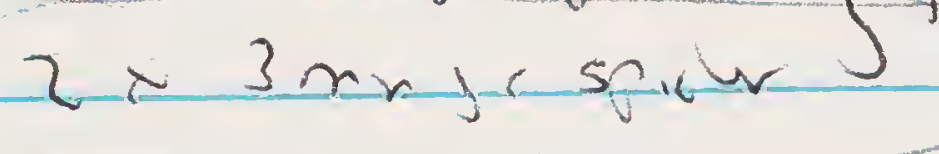

301

100

(1)

100

2oj lien gaen hip it

$20^{\circ}$

3oj at b

$1 / 8$
No incedetio

toung forest 2/16

cin: to ? , h !

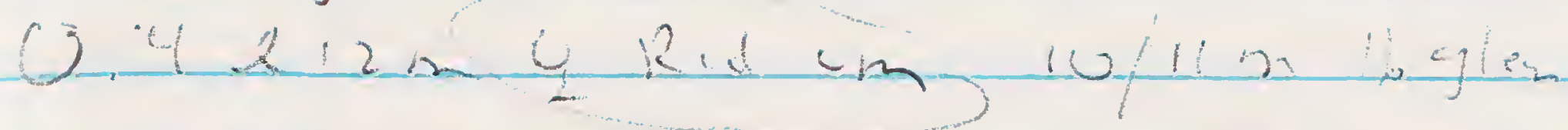

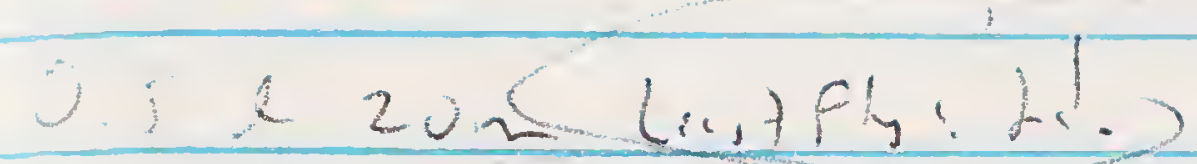

$$
3 \times 5^{-1}
$$

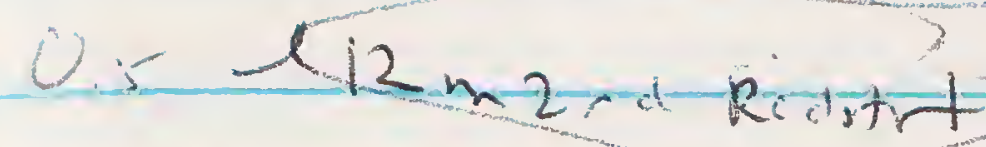

0 in $A_{n}+$ trajes

$0.3+6,20$ wather

1.2 esin Relitirt

1.5 R17 m WeU h

Y.nW

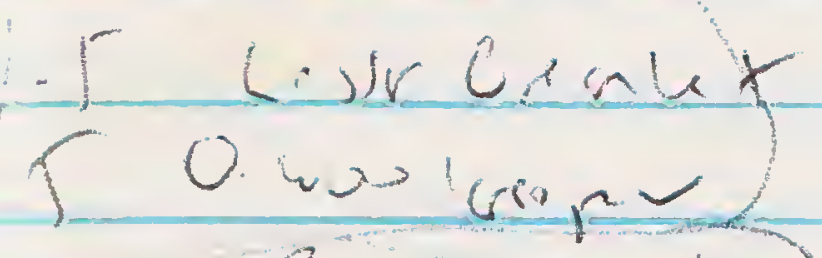

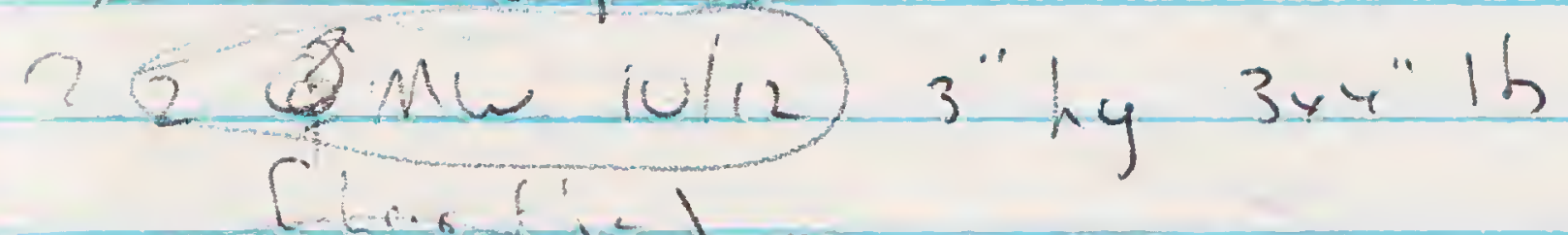

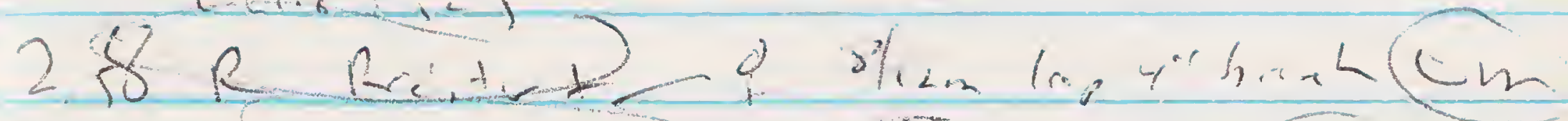

3.223 .2 e 10 in MWP/irin

2. Ke 15m min 1972 m by $4 \times 6$ "

$35 R$ tw $h$

4. $F$. B hum Li?

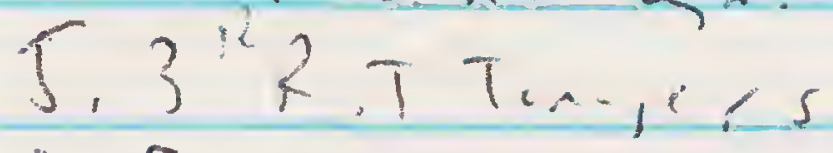

G. Sridb, :

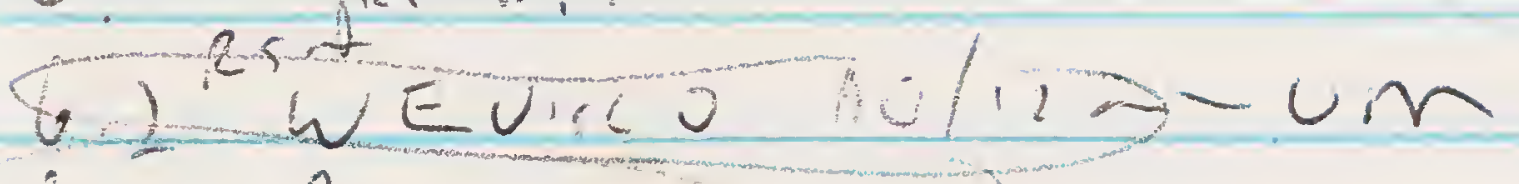

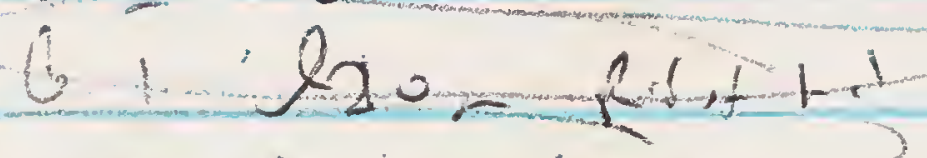

C.) l $5 m$ Leffita.

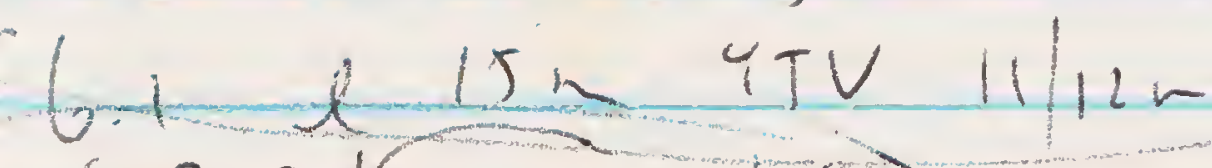

$63 R$ thw in

Uu2i,uts

molehoros 


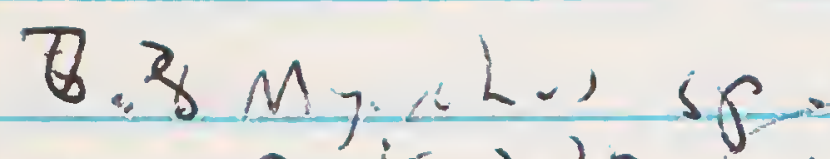

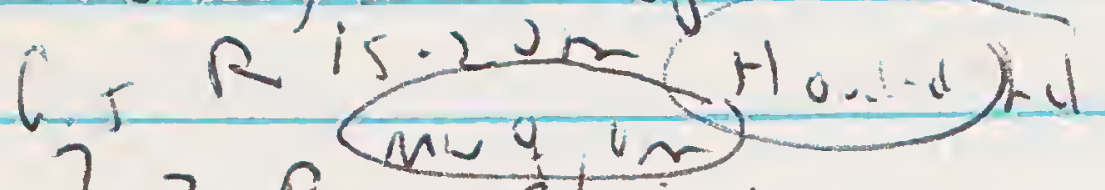

$7.3 R^{\mathrm{mat}} \mathrm{s} / \mathrm{m} \mathrm{m}$

104 l $3 \mathrm{~m}$ Rid imm

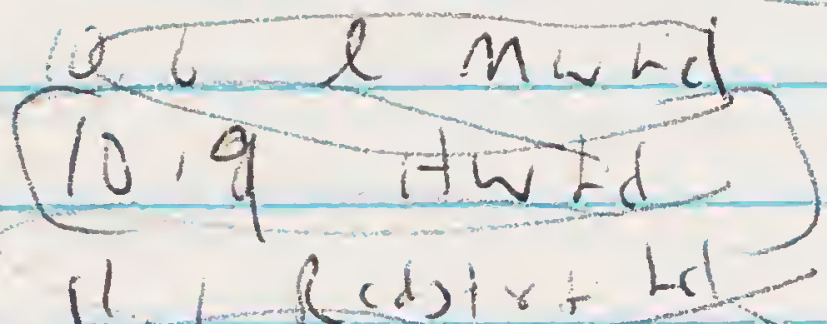

$$
\begin{aligned}
& 16.8^{2} 3_{0} \text { The het }
\end{aligned}
$$

12. Livcezut

12C R 10, MLLW

4 a $12 n 3 H_{\text {und ad }}$ )

19: 4 F.B.h.monr

$17.6<e^{1 n+3 \pi} 6 / x$

onc bodei (1,1)hads?)

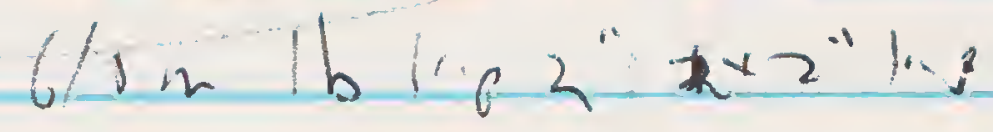

$$
\begin{aligned}
& L=2 \\
& \theta+1=8 \\
& \text { Hu }=6 \\
& \text { prin }=9 \\
& \left.\begin{array}{l}
w^{-T}=2 \\
T-1
\end{array}\right)
\end{aligned}
$$

24

$2^{3}$

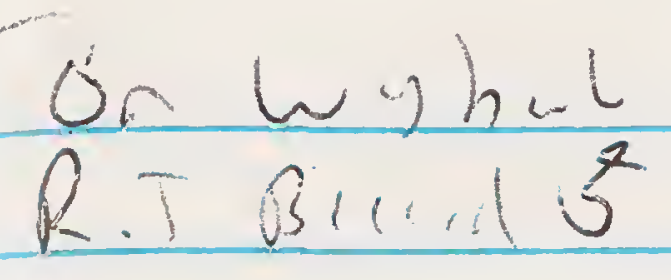

NI.S UM WEU

7.5 OLB WEV E

5.5 Honded 5 Red?

$B+\omega b-L$

Mw qu

Alogaty pala ibgillapec

$4 / 4.5 \ln$

BT $\mathrm{C}$

$$
4+56
$$

$11 / 3$ an lblip $3^{1}$ 
900 injatconss lim

$7 R$

(1) $\sin$ nophit

$100<3 m$ hinlth

100 maspid

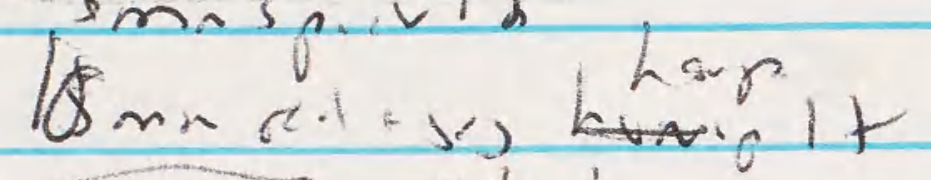

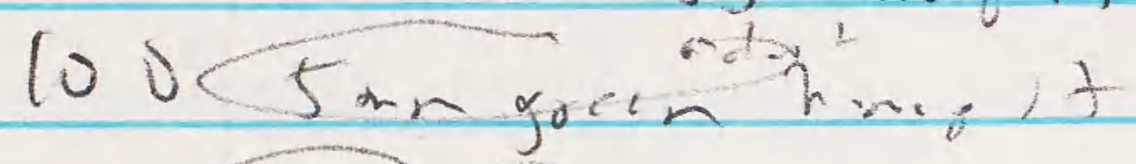

1003 (4massiarstb

200

102

$2003 w+2 i n k$

$3 m+8$ ?

$10 i$

$100-3$ mastertingts

Unr, ar, on

No

300

$2 / 1$

1,

is

los $-(3 m-3,2) / h$

100

103

$1003 m n$

tmn g giv/h w) $330-$

Gunde A,-il opposte dretion

ma nomel un rentril

queala

inin 5 Ma,rulia

of Reditert

6 Prola

Gernith Elcen in

GPolla - flom vine nod

\& R i.

mw

$2003 m_{n}$ henth

2oo 
Milo

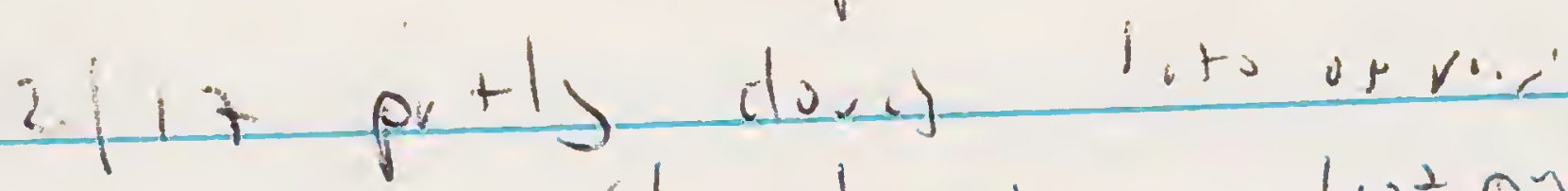

cin hind $10^{2}$ and

7. $8 \overrightarrow{4}, T$

$$
\begin{aligned}
& 0.1 e^{5 \pi T} \\
& 0.1 \text { e? La. Flytide } \\
& 2.325 x \text { Lrbs } 3 \text {, for he fure } \\
& \text { Hounderen sirgin? }
\end{aligned}
$$

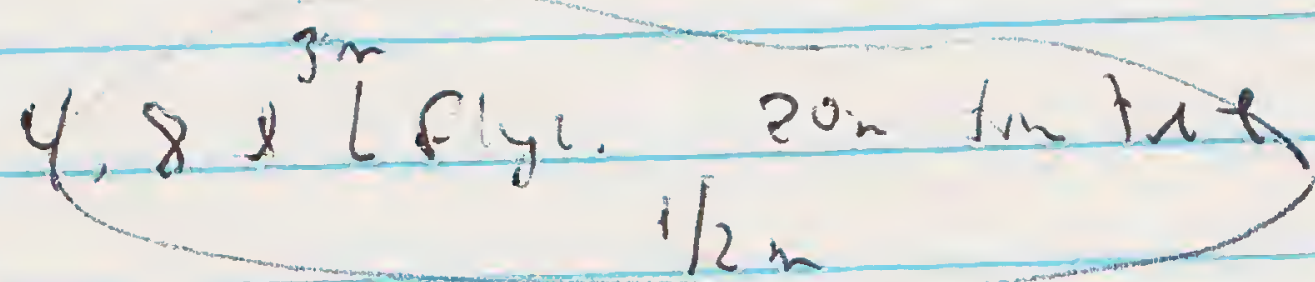

4.3. (mw:in)

$$
-4,20.2 / 1 \text { m Sinturio }
$$$$
6,4 R 0.3 p \text { icous }
$$

$$
\begin{aligned}
& 7,2 \text { RB G, D P C } \\
& \text { W. } T \text { Bow }
\end{aligned}
$$

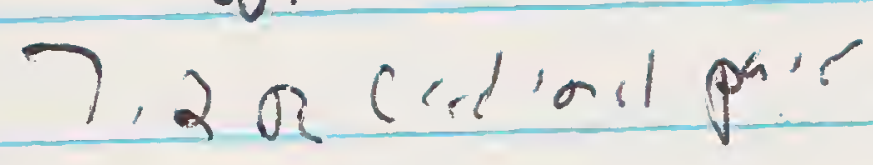

$$
\begin{aligned}
& \text { 7.3 (Riun m:pelal } 0 \text { ) } \\
& \text { e. } 1
\end{aligned}
$$

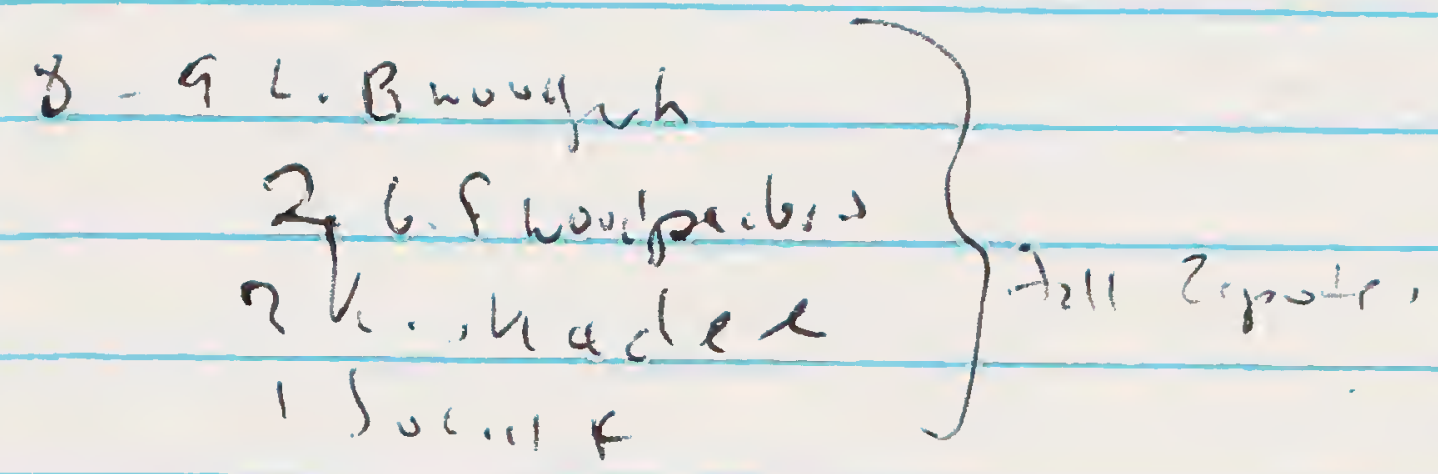

$$
\begin{aligned}
& \text { previn Blalbid }
\end{aligned}
$$

Solity Jidrepr uudrec

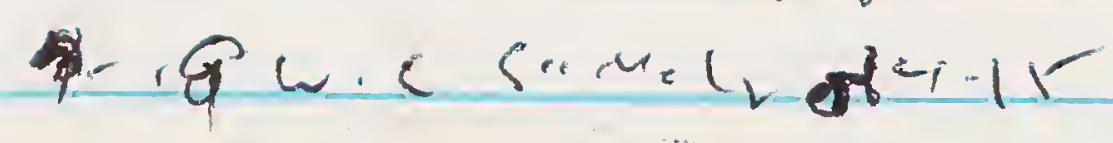

$$
\begin{aligned}
& 9-16 \text { i + bे a fece }
\end{aligned}
$$

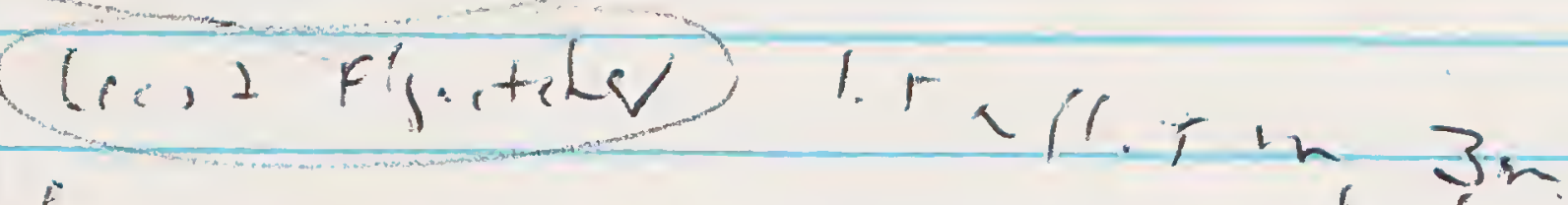

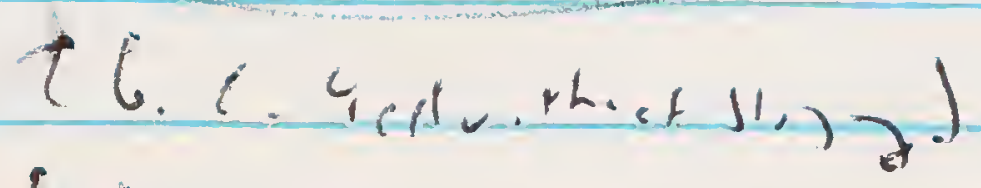

$$
\begin{aligned}
& \text { E. } \\
& \text { holiceter }
\end{aligned}
$$

$$
\begin{aligned}
& \text { 10-11 wieseed. } \\
& 14.1625 .410 \text { ? } \\
& 11 x^{2} \text { BElcea; } \\
& 17,+y, f, 6, s, 9,-1 \\
& 14.9 \text { \& 6.s.nengo } \\
& 12.5 \text { e o.pirrue } \\
& 13.44 \text { \& Gospoi }
\end{aligned}
$$




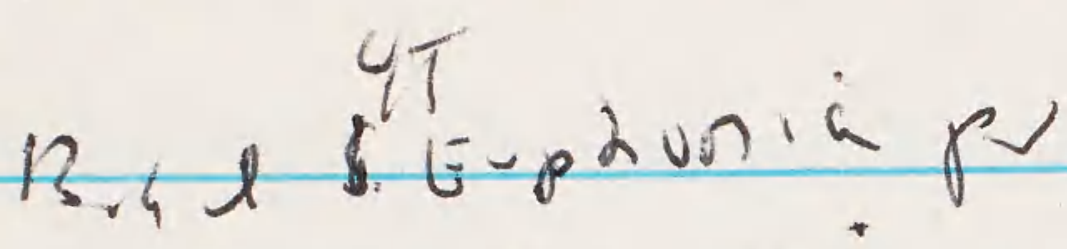

$13,820 R$ B.6. Grititad

B, p pro. pisend

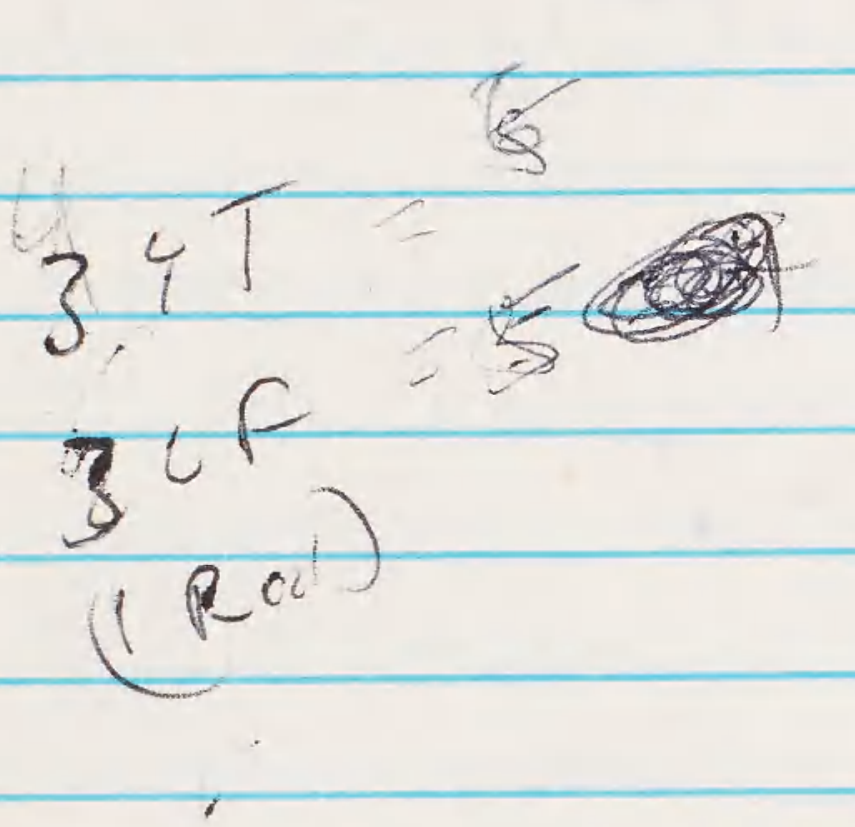

Sn Rd NW -

of Prile in topome flows

$$
\text { ii if } x^{\prime} \text {, }
$$

of fulle narl y fors

of ma low fortod

3 pule smill write flovers

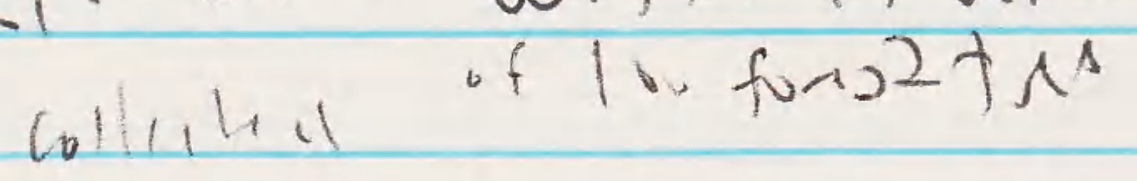

InWW 7ion torest

2/19

R.2y-Acyul ad S Ral. 


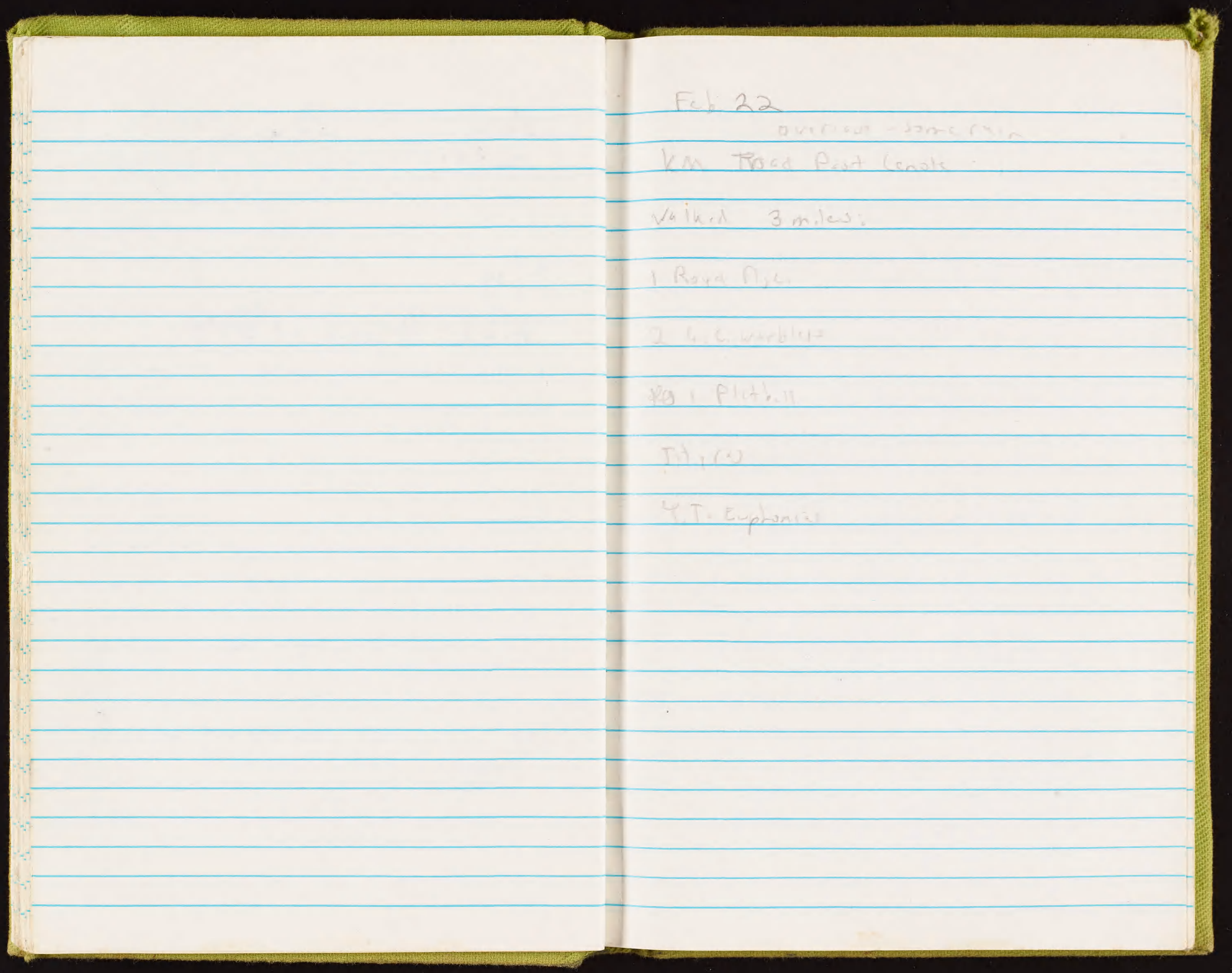




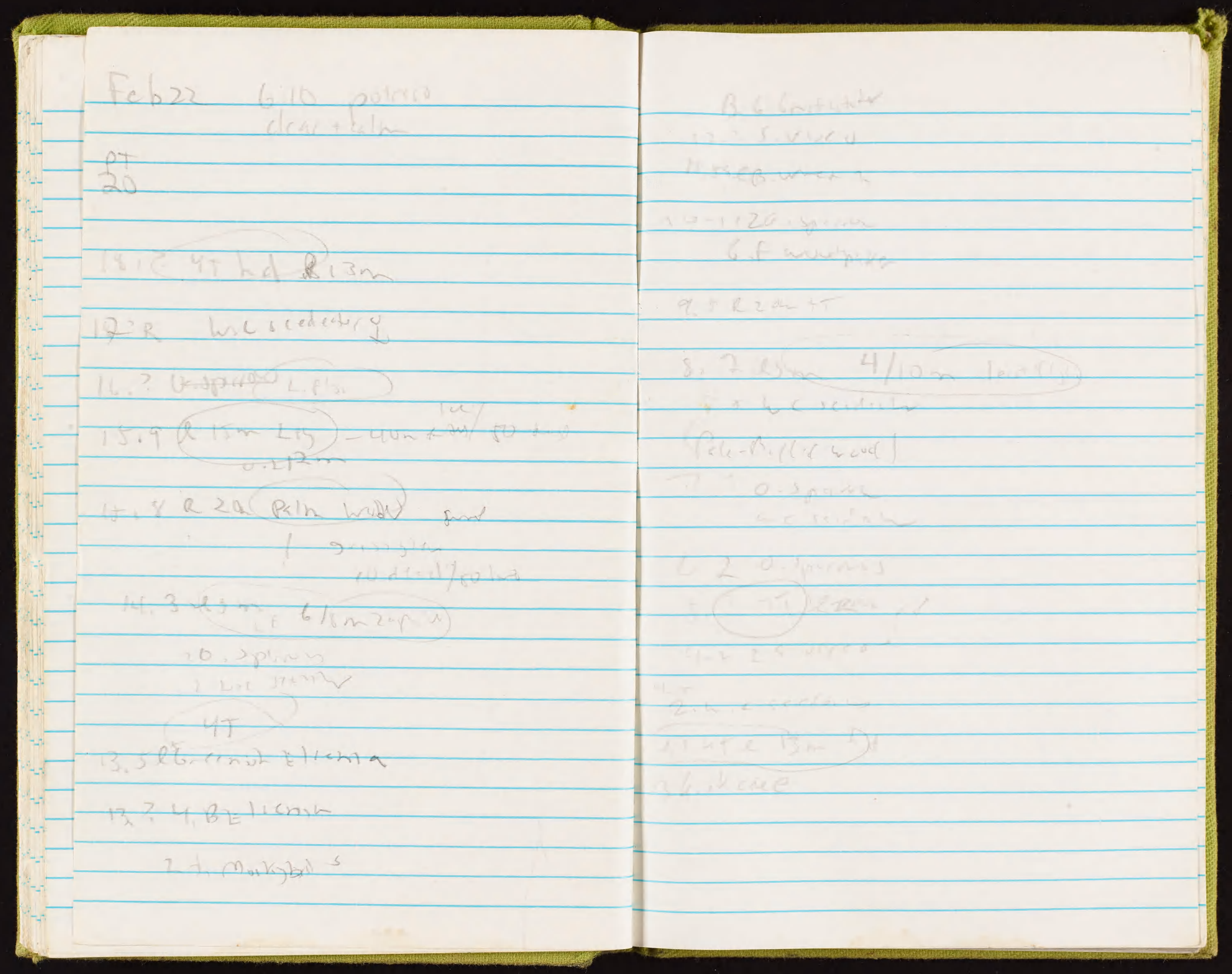



2123 . Milpa trandect

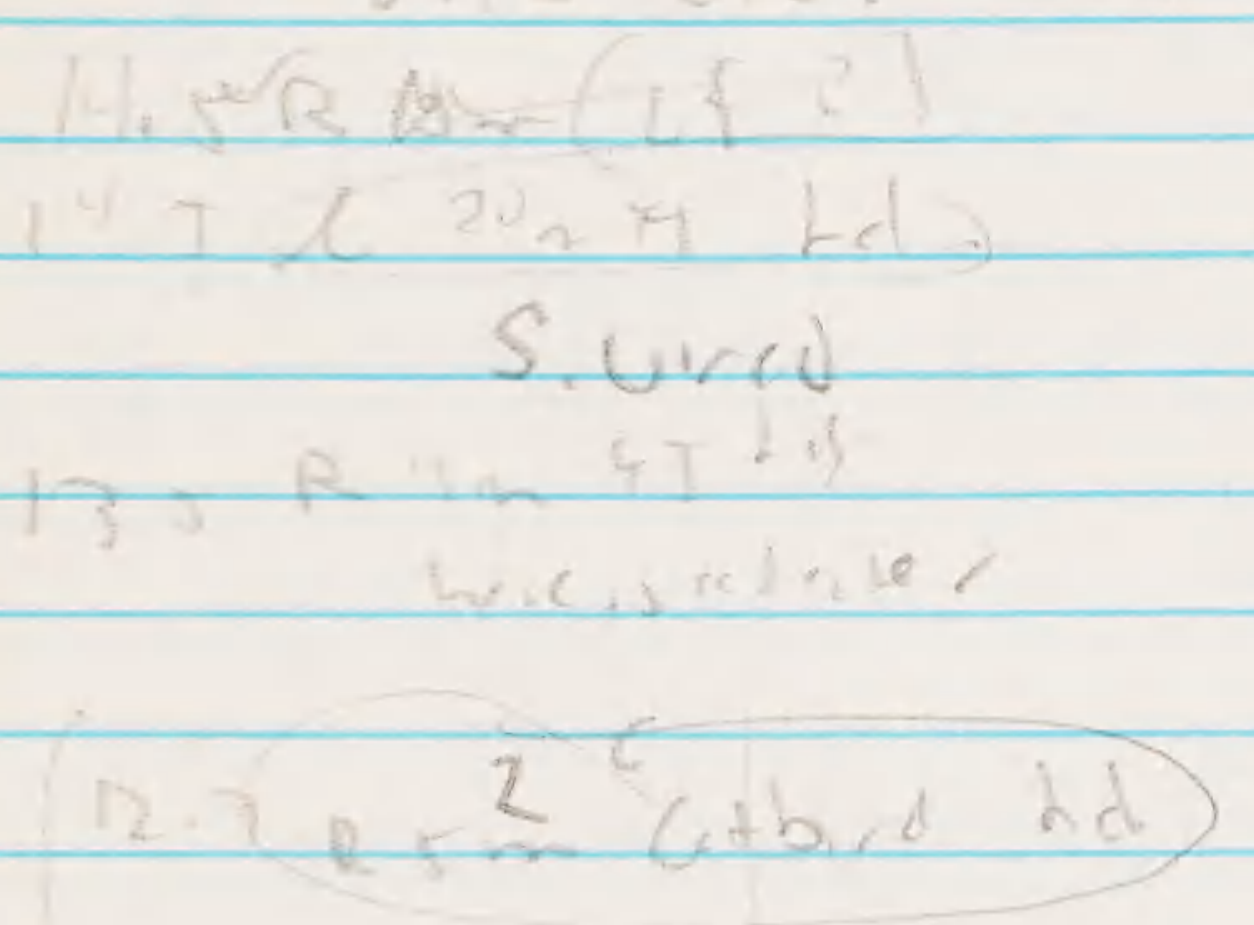

13 B.G,Gritan

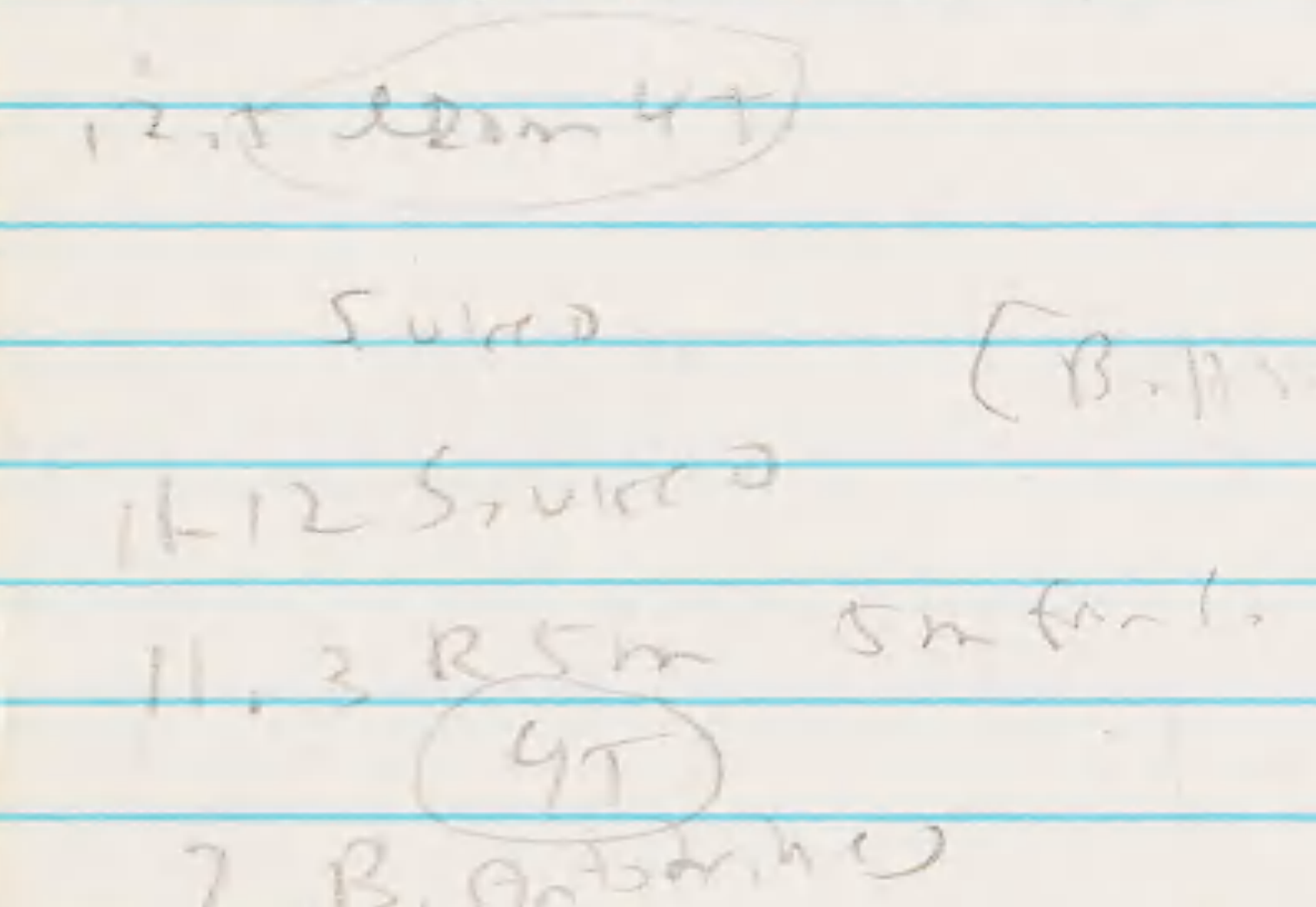

2 B. Antorinc

$4 \beta+1+x^{2}$

11.3 .0 o Red 0.2 $/ 7 \mathrm{~m}$

ib) a

$2+5$ fon $D_{n+1}$ wh

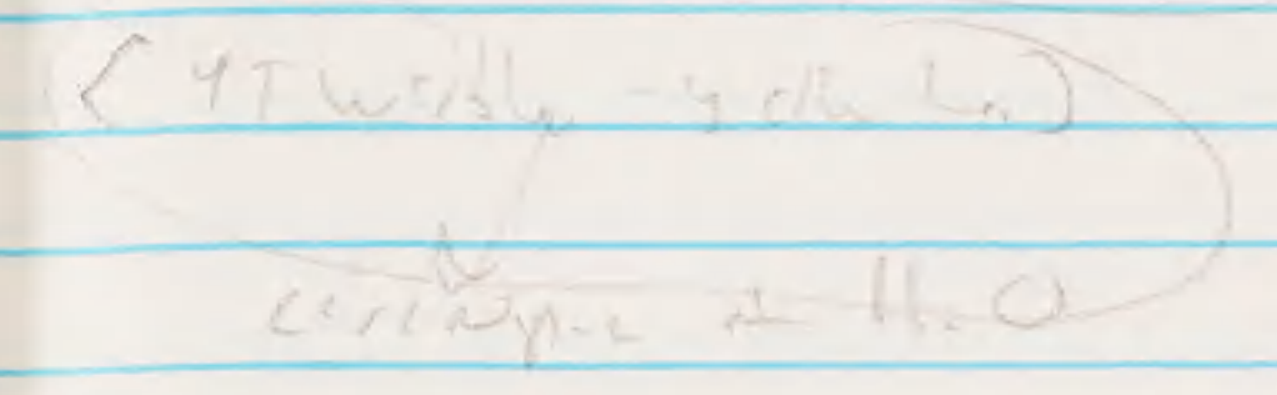

Res vencter

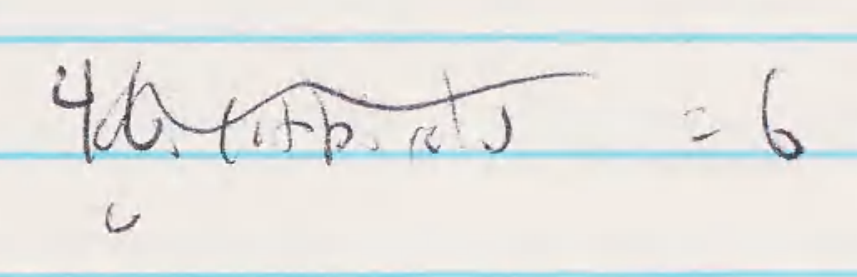

$\rightarrow$ M MW potiv<smiles>C1CC1</smiles> 


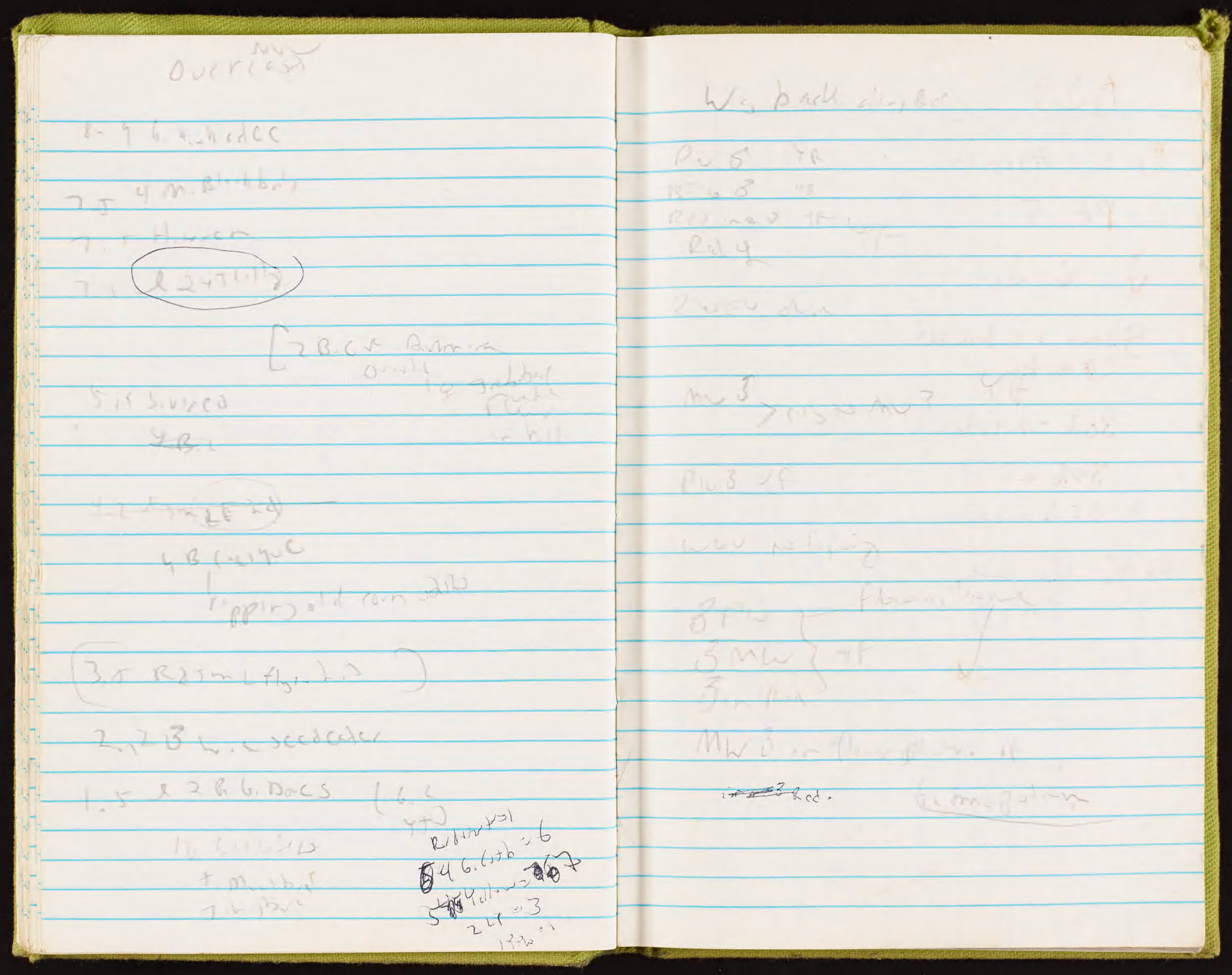


050

Precestimith

pd 5

$\downarrow \downarrow \jmath$

$12 \sin x+1 / 4 x+x$

2 to fin

$$
\begin{aligned}
& \text { 30)-shabater } \\
& \text { 34ho 6.C.? } \\
& 35 \text { s rocoue } \\
& B d \rightarrow h_{0} h_{0}+h_{1} \text { ) } \\
& \text { (helloti) }
\end{aligned}
$$

Vegctation surucy

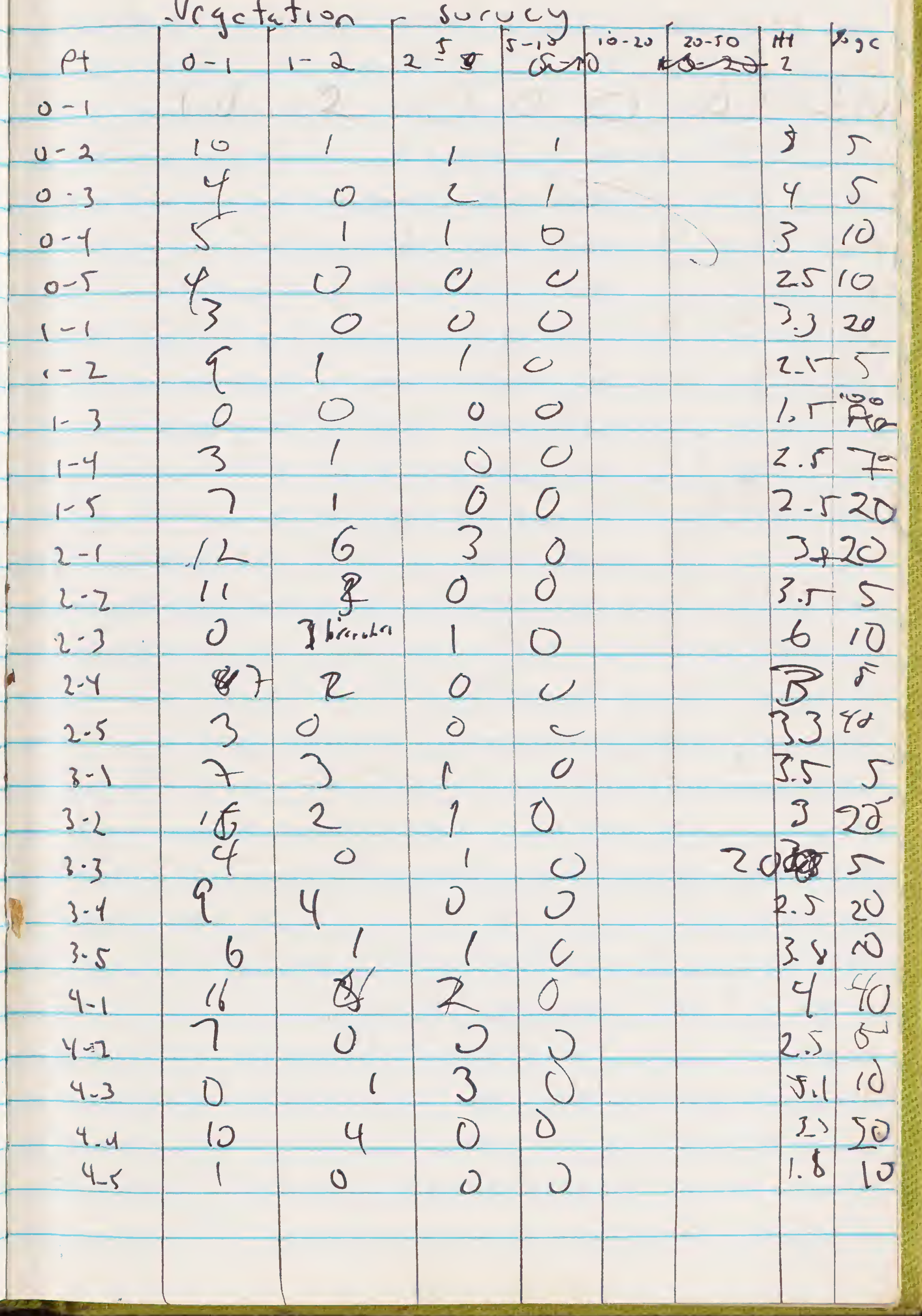




\begin{tabular}{|c|c|c|c|c|c|c|c|c|c|c|c|c|c|c|}
\hline & & 0 & 0 & & & & & 4 & & i] & 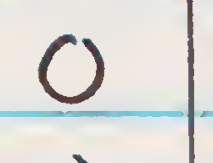 & & & \\
\hline & 10 & 0 & of & 1 & 0 & $\frac{18}{10}$ & $\begin{array}{c}10.2 \\
100-3\end{array}$ & 10 & $\begin{array}{l}0 \\
0\end{array}$ & $\begin{array}{l}1 \\
0\end{array}$ & $\begin{array}{c}0 \\
1\end{array}$ & $\begin{array}{l}0 \\
0\end{array}$ & & $6^{2}$ \\
\hline & & 0 & & 0 & Q & & 10.4 & 0 & & & 0 & & & 3.5 \\
\hline & 2 & u & 0 & U & & & .0 .5 & ? & 0 & 1 & & & & \\
\hline & $\begin{array}{l}8 \\
9\end{array}$ & 6 & 0 & 0 & 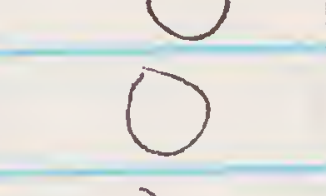 & 3.70 & $\begin{array}{l}11-1 \\
11-2\end{array}$ & 7 & 1 & $\begin{array}{l}0 \\
0 \\
0\end{array}$ & 0 & 0 & & \\
\hline & 3 & J & 0 & 0 & & 20 & 111.3 & 1 & $u$ & u & & & & \\
\hline & $\frac{5}{4}$ & 1 & 0 & $\begin{array}{l}u \\
u\end{array}$ & 0 & 35 & $11-4$ & $\frac{0}{2}$ & 0 & & & $\sigma$ & & \\
\hline & $x \cos$ & 0 & 5 & & 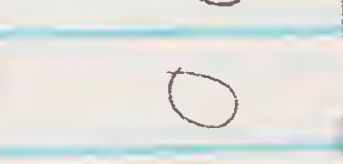 & 80 & $12-5$ & 1. & $u$ & $u$ & $u$ & & & \\
\hline & & $u$ & $u$ & $\checkmark$ & 0 & $2=30$ & $12-2$ & 0 & 0 & & & & & \\
\hline & $m^{2}$ & $\frac{u}{3}$ & 0 & 0 & & $\begin{array}{l}5.5 \\
3.5\end{array}$ & $\begin{array}{ll}12-3 \\
172-1\end{array}$ & $\begin{array}{c}14 \\
?\end{array}$ & 0 & , & 1 & U & $\begin{array}{l}0 \\
0\end{array}$ & \\
\hline & 18 & 21 & 0 & 0 & U & 4 & & $\gamma$ & 0 & & 0 & & & $\theta$ \\
\hline & 12 & $\begin{array}{lll}0 & 0 \\
0 & 0\end{array}$ & 0 & u & $\checkmark$ & 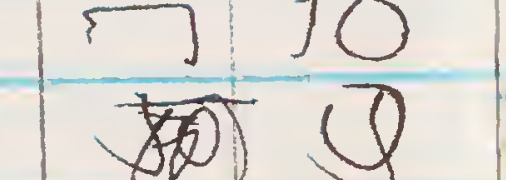 & $13-1$ & 28 & 1 & $\begin{array}{l}0 \\
0\end{array}$ & 1 & $u_{0}$ & & \\
\hline & 1 & 00 & 0 & 0 & $u$ & 010 & & (1) & 0 & 0 & 0 & & $c$ & \\
\hline & & & & & & 2.547 & $13 . y$ & & & & & & & \\
\hline & 8 & 2 & $\theta$ & 0 & 4 & 310 & 13.5 & 0. & 2 & U & 0 & 0 & & \\
\hline & 13 & 2 & u & $u$ & 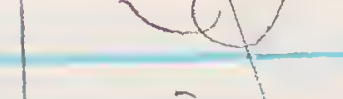 & & $10-1$ & 15 & 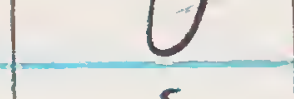 & & & & & \\
\hline & $a^{11}$ & (j) & 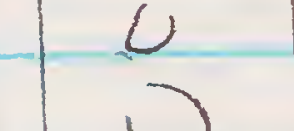 & & & & 18.2 & 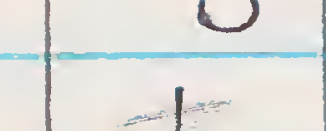 & 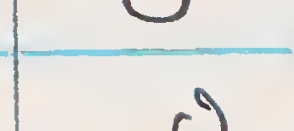 & $\sigma$ & 1 & 0 & & \\
\hline & 12 & $\frac{2}{2}$ & 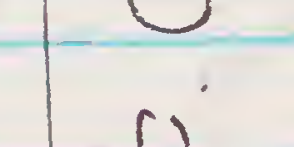 & 14 & & & & 3 & & (1) & & & & \\
\hline & & 0 & 0 & & & 315 & 14.5 & 8 & 1 & 0 & $j$ & 0 & 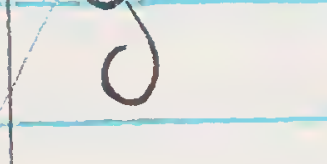 & \\
\hline & 4 & & & & & & & & & & & & & \\
\hline & & & & & & & & & & & & & & \\
\hline
\end{tabular}




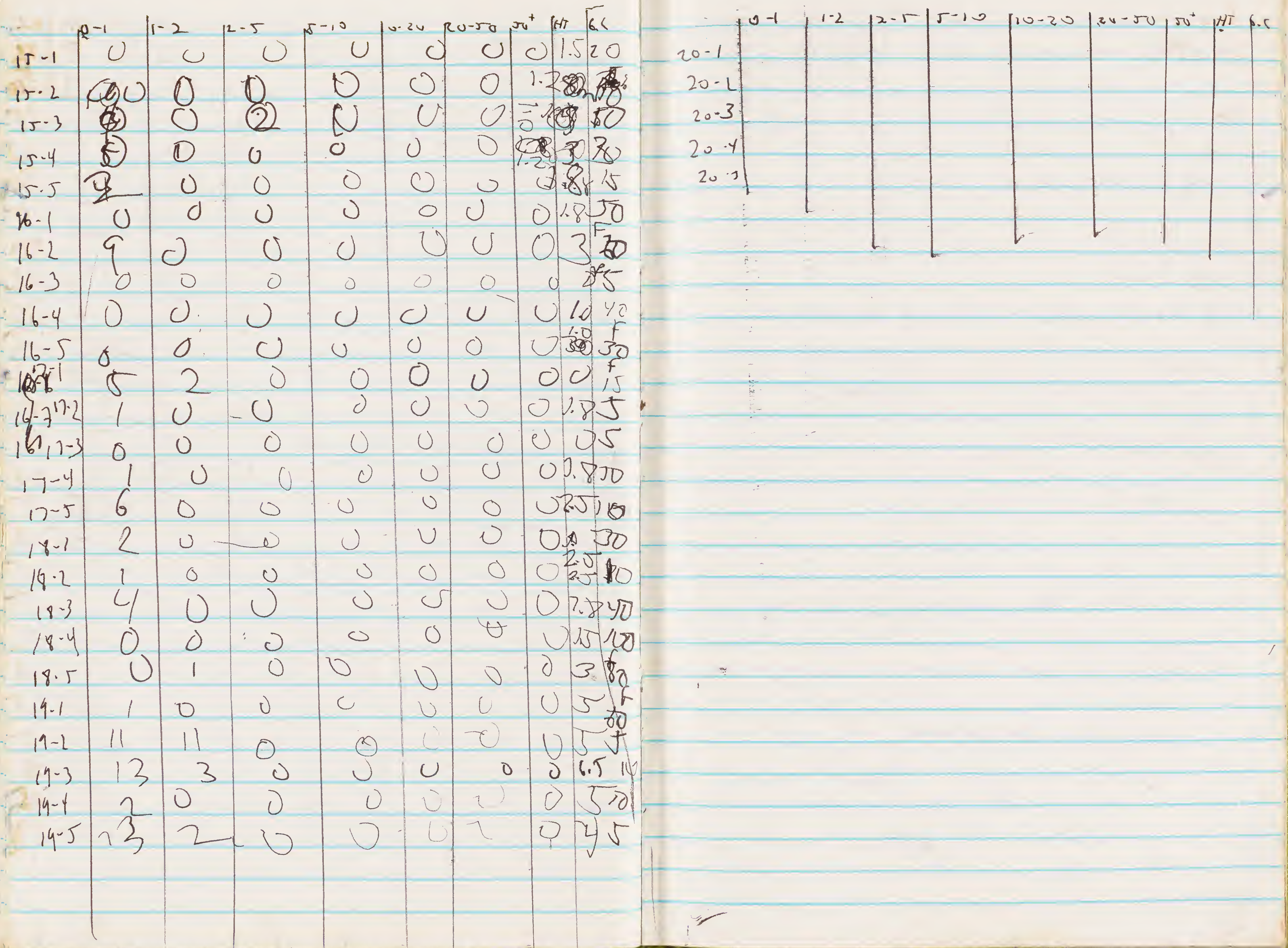


ungrazed araJuas

Fib 27

Rdilun $S$

unz bind low torest clere cool

$\log _{5} 3$

$2 \cdot s^{l e d}$

q troded - berk

ad 3 Red

lot of $R \cdot x-1-t h c h$

(b) Hood

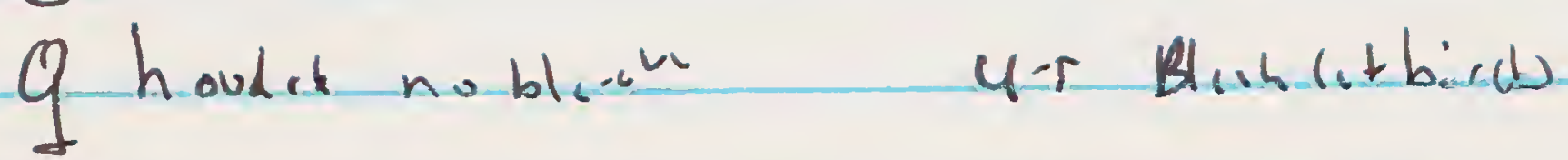

mw

$4 x-h-v_{n}=$

SYT

U.B wren

2 Ningardwos

lousbirk

$V$ M 35 krol tron propluse $\approx 2-34 m$

(2) frost + Iquateted areds)

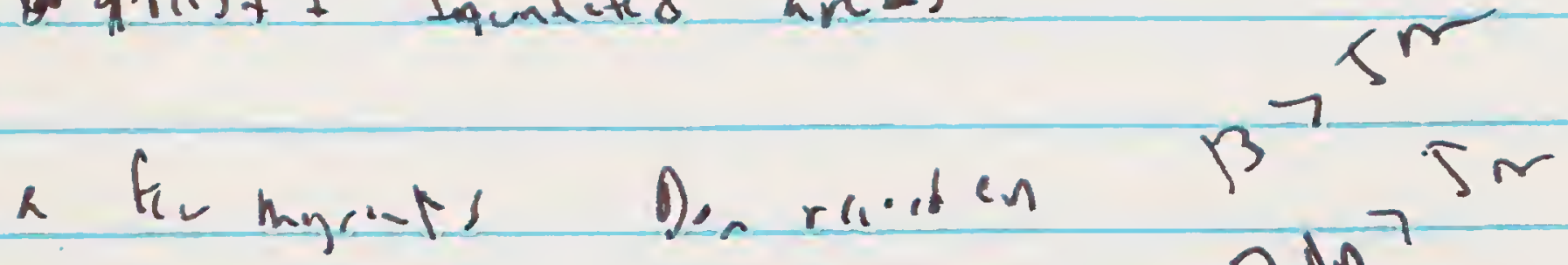

3572669
P) $0-1,1-2$

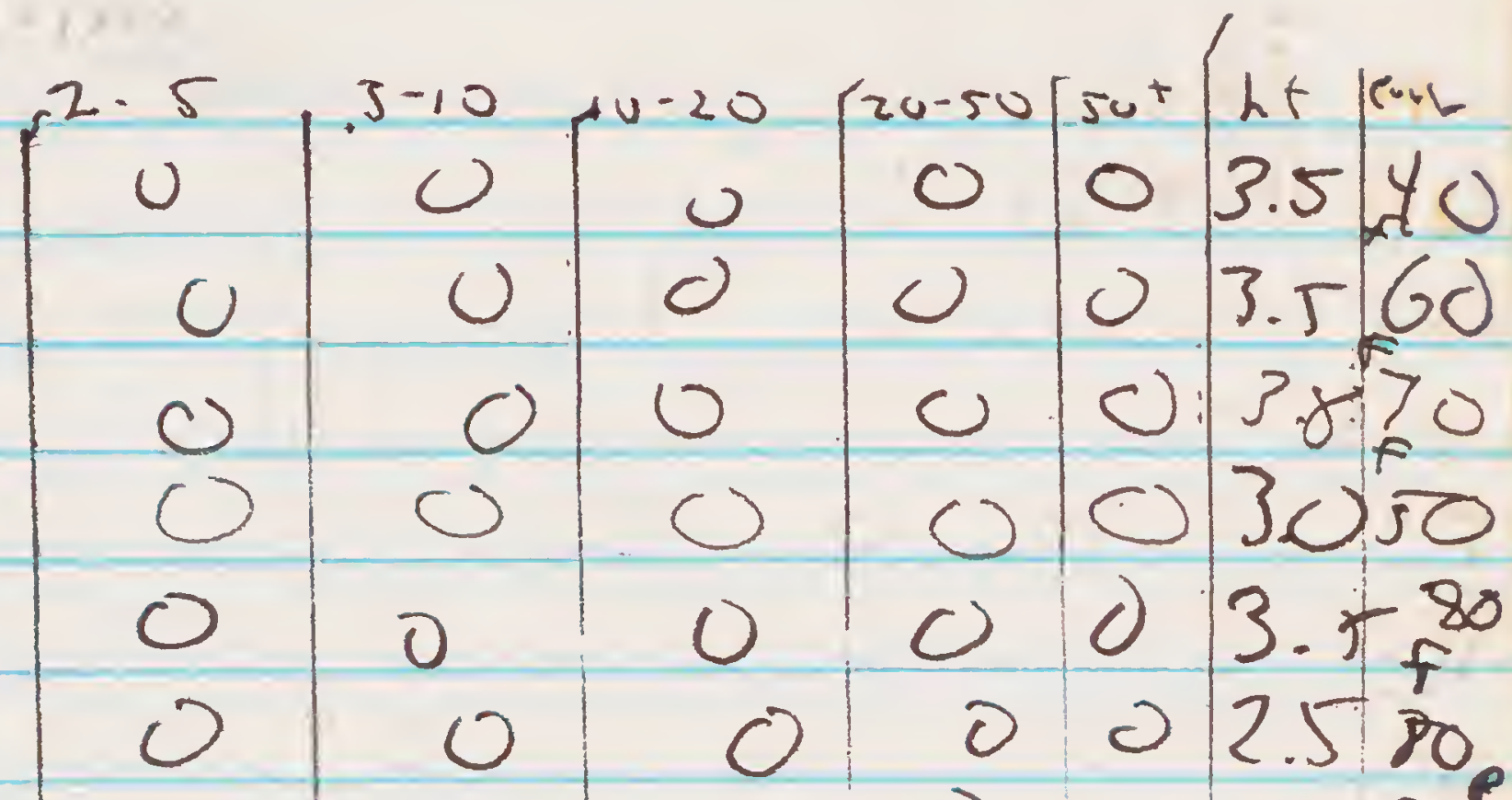

\begin{tabular}{c|c|c|}
$1-1$ & 7 & 0 \\
$1-2$ & 5 & 2 \\
$1-3$ & 0 & 0 \\
$1-4$ & 0 & 2
\end{tabular}

1

$\gamma$

$\begin{array}{ccccc}0 & 0 & 0 & 3.290^{\circ} \\ 0 & 0 & 0 & 3.865 p \\ 0 & 0 & 0 & 3.580 \\ 0 & 0 & 0 & 3.828 \\ 0 & 0 & 0 & 3.70\end{array}$

16

2

\begin{tabular}{l|lllllll}
0 & 0 & 0 & 0 & 0 & 0 & 3 & 70 \\
0 & 0 & 0 & 0 & 0 & 4.250
\end{tabular}

$\begin{array}{llllll}0 & 0 & 0 & 0 & 02.08- \\ 0 & 0 & 0 & 0 & 04.230\end{array}$

$c$

c 0

C) 0 \& 62

C 04, .

- C 2.2 ह

$\begin{array}{lllll}0 & 0 & 1.3 & 1.0 \\ T_{0}^{\circ}\end{array}$

$0003 T_{k}^{T *}$

\begin{tabular}{l|l|l|ll}
0 & 0 & 0 & 0 & $0.7 .7-t$ \\
0 & 0 & 0 & 0 & 0 \\
0.240
\end{tabular}

$\begin{array}{llllll}0 & 0 & 0 & 0 & 0 & 0\end{array}$

2

$\begin{array}{lllllll}1 & 0 & 0 & 0 & 0 & 48 & f_{0}^{0} \\ 0 & 0 & 0 & 0 & 0 & 4.2 \%\end{array}$ 


\begin{tabular}{|c|c|c|c|c|c|c|c|c|c|c|c|c|c|}
\hline Pt & $\Leftrightarrow \quad 0<$ & & & 1.200 & r & or int kase, & & & $\begin{array}{l}0-2 \\
2 \cdot-15\end{array}$ & 25 & -10 & 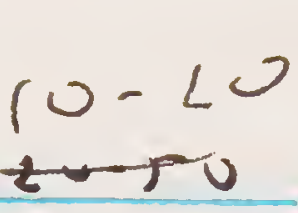 & 20 \\
\hline $3-1$ & 4.2 & $\delta$ & 0 & 0 & v & $048.2 \quad 30$ & $10-1$ & & 3 & 0 & $\partial$ & 0 & $0 \int_{4}^{4}$ \\
\hline 5.2 & 00 & 1 & 1 & 0 & $u$ & $v b$ & 0.2 & 12 & 1 & 1 & 0 & 0 & $0 \% 2$ \\
\hline 5.3 & 41 & 0 & 0 & $u$ & U & 0.7 & 10.3 & 8 & 1 & 0 & 0 & 0 & 04. \\
\hline $5-4$ & 00 & 0 & 1 & 0 & 0 & 06.5 & $10^{-4}$ & 1 & 0 & 0 & 0 & 0 & 02 \\
\hline $5 \cdot 5$ & $2 !$ & c) & ( & $\cup$ & 0 & 06 & $10-5$ & 0 & 0 & U & 0 & 0 & 0 \\
\hline $6-1$ & 00 & 1 & 0 & 0 & c) & 057 & $1-1$ & 11 & 2 & 0 & 0 & 0 & 04 \\
\hline $6-2$ & 162 & 2 & 0 & 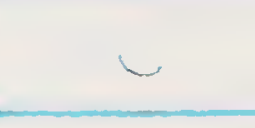 & 0 & 15.50 & $11-2$ & 4 & 0 & 0 & 0 & 0 & 07 \\
\hline $6-3$ & 70000 & 1 & 0 & 0 & & 03.5 & $11-3$ & 0 & $c$ & 0 & 0 & 0 & 02 \\
\hline $6-4$ & 1324 & $i$ & v & 0 & & 8 & $11-4$ & 10 & 3 & 3 & 0 & U & 010 \\
\hline 6.5 & & 0 & 0 & v & $u$ & 0 & $-1)^{-5}$ & 19 & 2 & 2 & 0 & 0 & 08 \\
\hline $2-1$ & 110 & 0 & 0 & 0 & 0 & $1.9 \%$ & $12-1$ & 3 & 0 & $\alpha$ & $J$ & 0 & 04 \\
\hline $1-2$ & 11 & 0 & 6 & 1 & 0 & $\theta 8$ & -2.2 & $\gamma$ & 2 & 0 & 0 & $c$ & 05 \\
\hline 2.3 & 253 & 0 & (6) & 0 & & $\begin{array}{llll}0 & 3.5 & 10\end{array}$ & $(2-3$ & (u & 4 & 0 & 0 & 0 & \\
\hline $7-4$ & 10 & (a) & 0 & U & & $\begin{array}{ll}0 & 2.29\end{array}$ & $12-4$ & $C G$ & 0 & 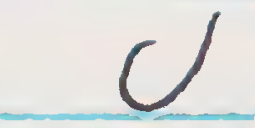 & $\circlearrowleft$ & $c$ & \\
\hline$y-2$ & 10 & 1 & 0 & 0 & 0 & 07 & $12-5$ & 0. 1 & H! & oust $k$ & -0 & & 10 \\
\hline 9.1 & Do 0 & 1 & 0 & 0 & $\cup$ & 08 & $13-1$ & 4 & 1 & 1 & 0 & 0 & d \\
\hline 4.2 & 162 & 0 & $\partial$ & U & U & (v) 5 & $13-2$ & 0 & 0 & C) & U & 0 & $u^{\operatorname{lm}}$ \\
\hline 4.3 & 92 & 1 & 0 & 0 & U & $\cup 42$ & $13-3$ & 4 & 3 & 2 & 0 & 0 & $\alpha$ \\
\hline $8-4$ & 36 & 0 & 0 & 0 & U & 04.5 & $13-4$ & & & 2 & & & \\
\hline 8.2 & $5 \cdot 0$ & 0 & 0 & 0 & U & $4:$ & 13.5 & 1 & ( & 1 & 0 & $\mathcal{U}$ & 0 \\
\hline$a-1$ & $n=-2$ & 0 & 0 & 0 & $u$ & $0 \quad 3.2$ & $14-1$ & 4 & 0 & 1 & 0 & 1 & $Q$ \\
\hline$\{2$ & 72 & 0 & 0 & 0 & U & $(U)$ & wet & 7 & 1 & 1 & 0 & 0 & 0 \\
\hline $9-3$ & $9 \sqrt{2}$ & 2 & b & 0 & 0 & 0 & $14-3$ & $c$ & 0 & 1 & $\partial$ & 2 & 0 \\
\hline$\{-4$ & $10-1$ & 2 & U & $0=$ & (1) & 055 & $M-4$ & 0 & 0 & 0 & 0 & $U$ & $12^{8}$ \\
\hline$\{-5$ & 10 & 5 & 3 & 0 & 0 & $u-1.8$ g. $80_{1}^{\circ}$ & $i 4-5$ & & 0 & v & $U$ & U & \\
\hline
\end{tabular}




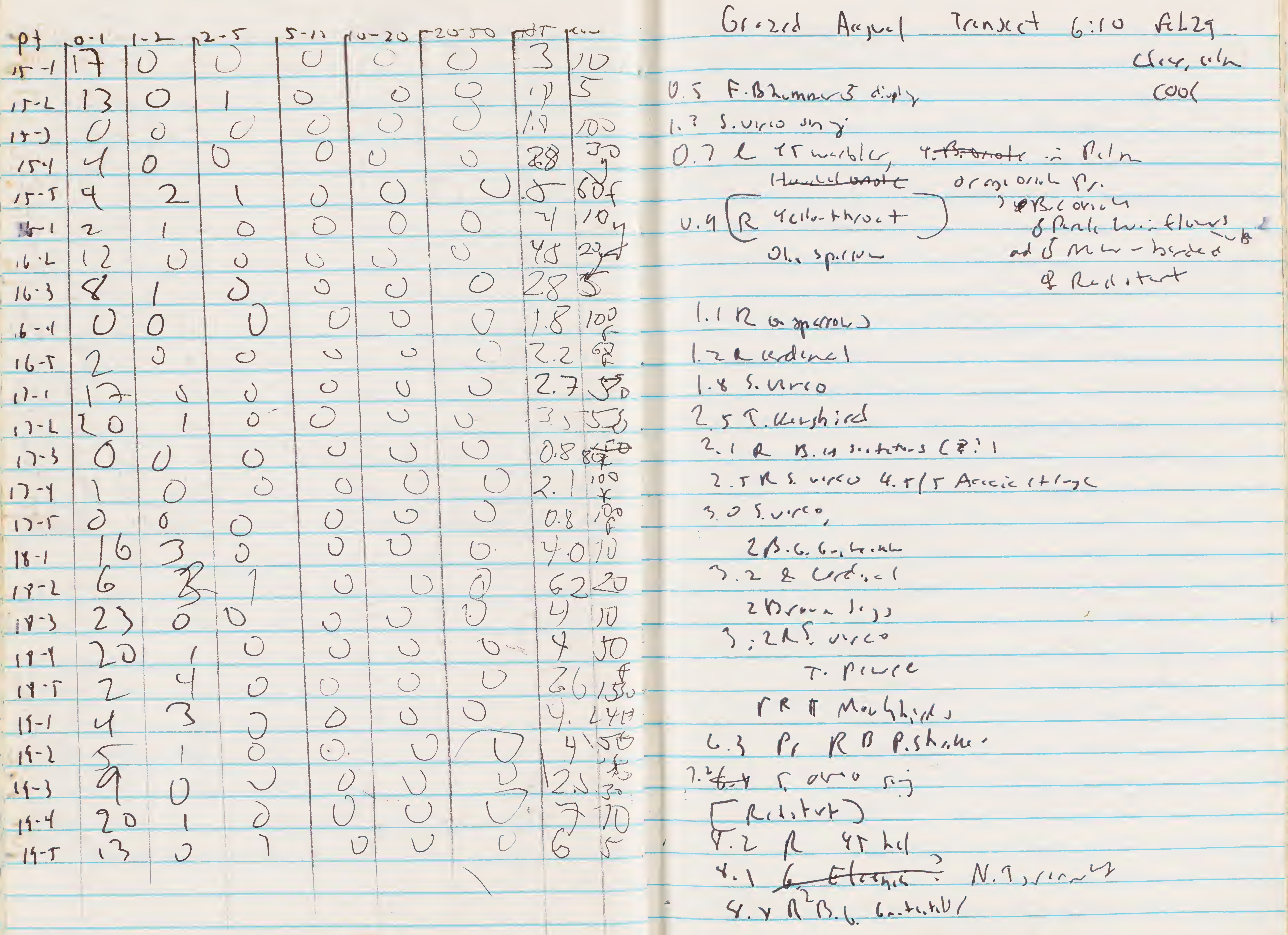


9.6 t. pacc

9. $* \cap$ S.urco

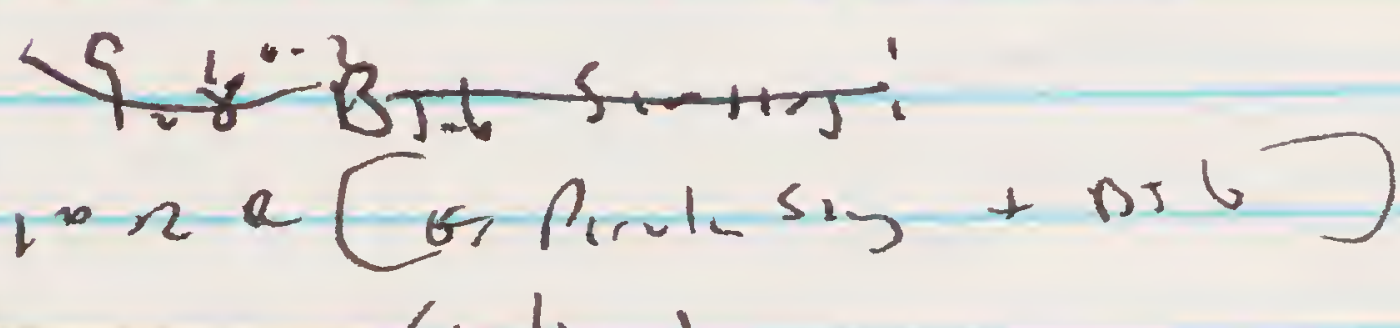

$$
\begin{aligned}
& \text { (evalue) }
\end{aligned}
$$

$9.2 \pi q$ gon $3 / 4 m$

flewpore

hort bivieco

lu.t canlin.

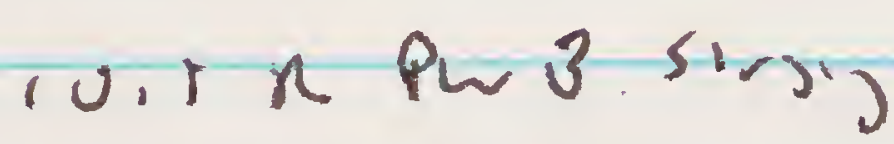

10.5 A PW

G. (1+bin)

11.15 .621400

HuLd

B, Antinu

uniusphil

Muhil

(11.) $1.12 \mathrm{~m} / 2 \mathrm{~m}$

12 I B.L Gnotide

$12.5+21234 T$

Riv y u.wole 1) S10

$$
\begin{aligned}
& 2 \text { r.phians } \\
& 2 \text { Senburcos } \\
& \text { 13.1 sure of? } \\
& \text { 13.2kirm Celienia } \\
& \text { panin q }
\end{aligned}
$$$$
\text { 13/4n }
$$

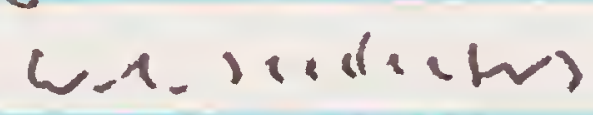

Cinnenenh-ma

15. ER B.6.6.titew

$17.5 \times 0.3 p+4$

15.6 s.u.reo

$$
\text { T-M.kblin }
$$

IC.1 2 more B.C. Lnetitchers

$1 \% .8 R$ MUy $\mathrm{im} / \mathrm{cm}$

LFFyc.

1r. 1 \& S.virec

[4Twathe : Poln uft thise...)

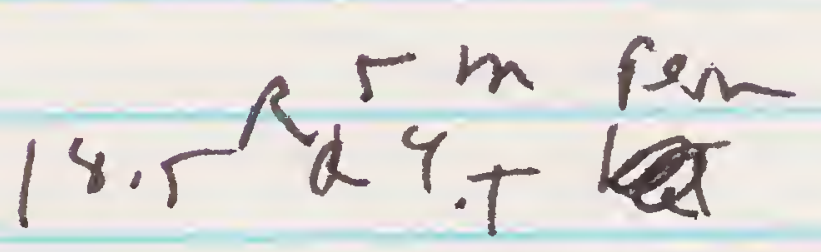

wh ionsicl

1.: 0.spersens

20 Pu flower red $4 / 6 \mathrm{~m}$

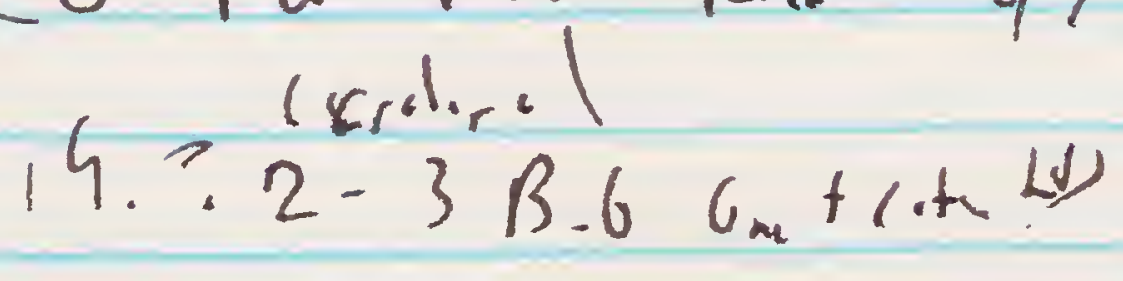




$$
\begin{aligned}
& M W=3 \\
& \cup F=2 \\
& \text { ष }=3 \\
& \psi_{\nabla} \omega=1 \\
& H \omega=1 \\
& p \omega=6 \\
& \text { Red }=1 \\
& \text { (it }=1 \\
& B 76=12 \\
& >19
\end{aligned}
$$

300

$$
\begin{aligned}
& 200 \\
& 200 \\
& 200
\end{aligned}
$$

Insect (r) PT D R $=45 m$

$0 / 1$ in icet

$$
\begin{aligned}
& 200 \\
& 100 \\
& 100 \\
& 100 \\
& 200 \\
& 1004 \mathrm{mn} \text { (1) } \\
& \frac{100}{100} \\
& \frac{200}{200} \\
& 100 \\
& 100 \\
& 100 \\
& 200 \\
& 70^{\circ}
\end{aligned}
$$


Old forist 3/1 6:20 dreste.n

0.0 e $7 m$ (mw h.l cill, to c..oth me ott

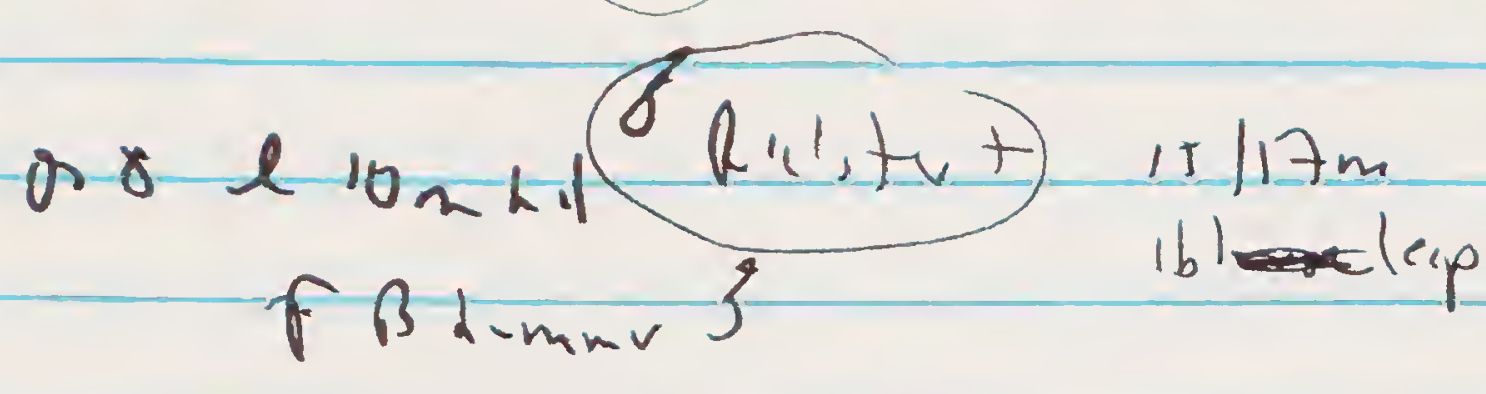

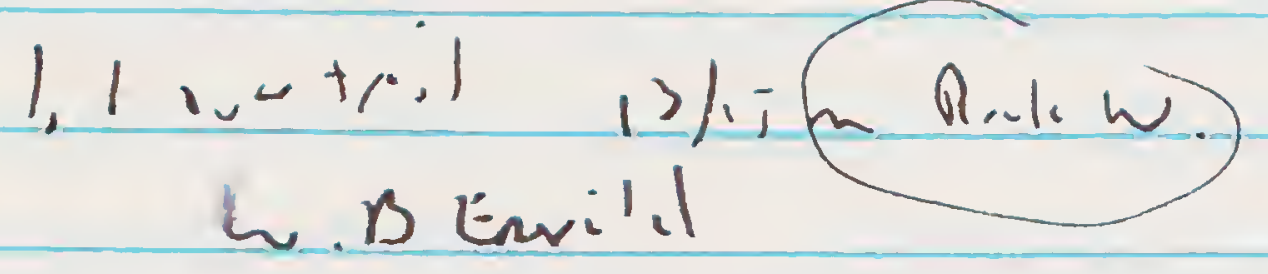

L, T Sihay:

Blur.B.ty N.,?

2? (. Ganht)

spilubiti

I. B noodreper

$2 \times R 20<q R D .5 / 7 n$

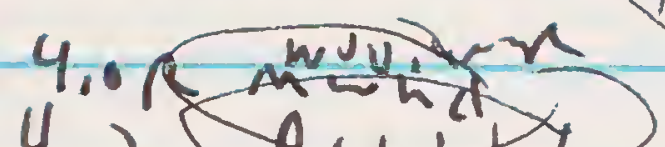

4. 2 (tintity

4.1) (mukil), 2 ane.kt?

B.h $+1,000$

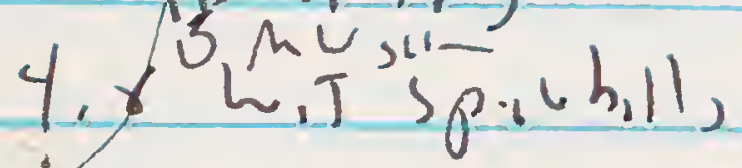

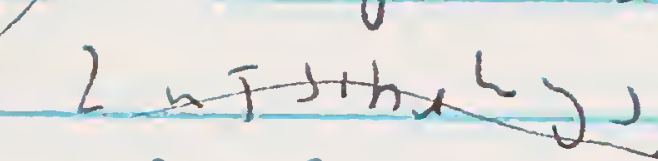

R11.tvthy-8a!

$R, T$ Tin, 2

tri....

J,3RLEU (2)

4T U sirsiz inti3-

j

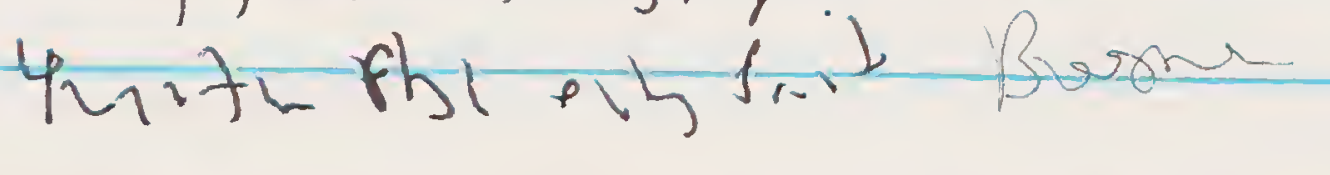

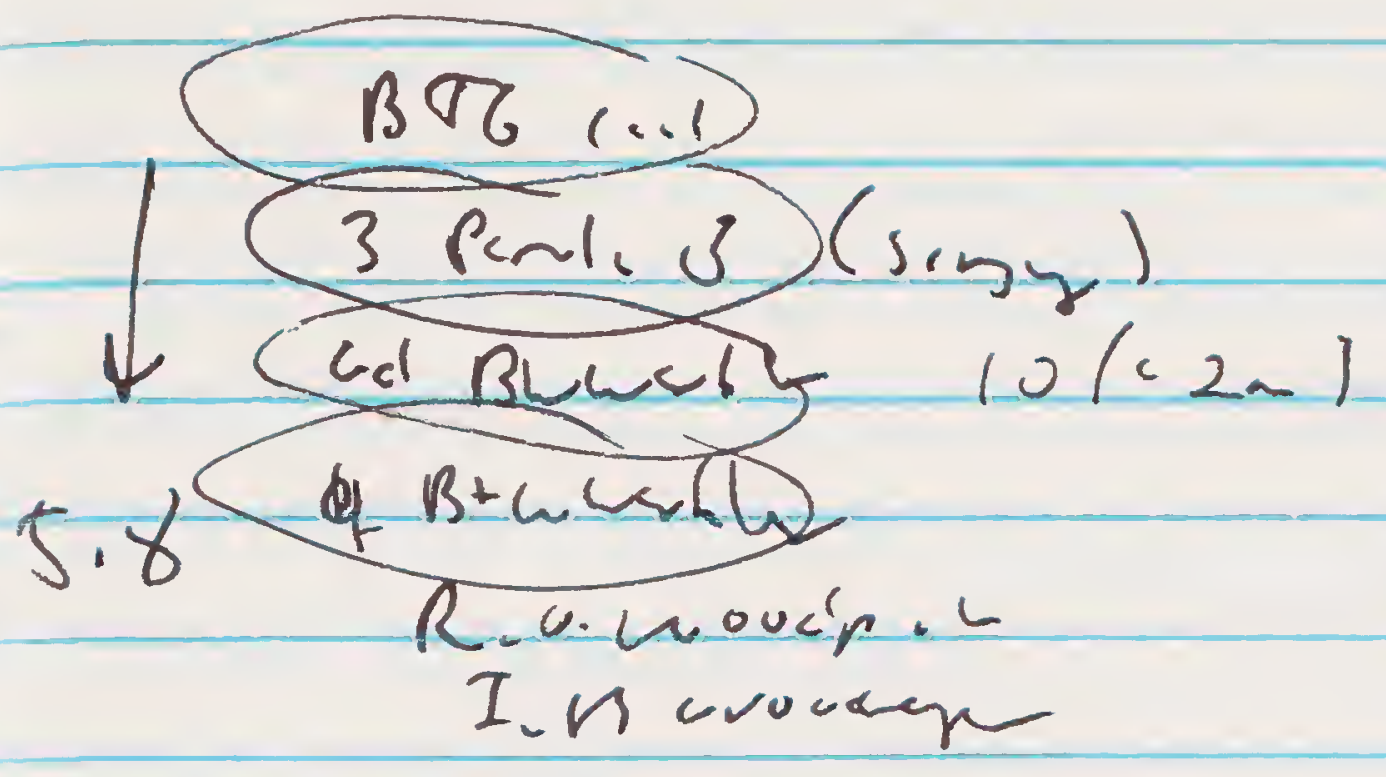

1.8 Exploteas

8.1 R makhel. D.c. 8 Alyc-

ORProliwh hl

9. Thentrito

T.IR $15 \mathrm{~m}$ od S R.C.

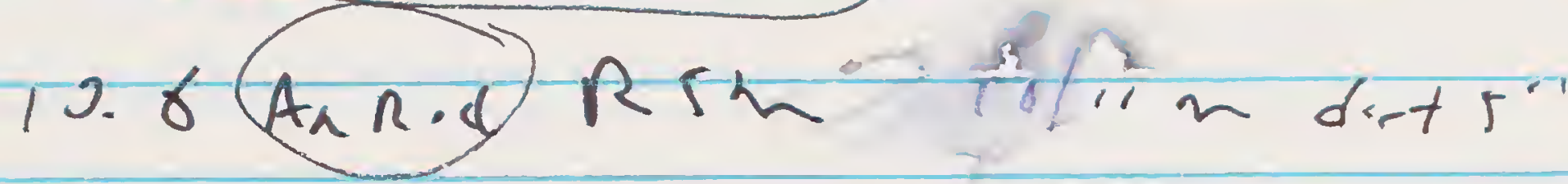

Oacch 1

$11.2 R F \sim n k w d$

1) $l \mathrm{ml}$

12 4.T tayk

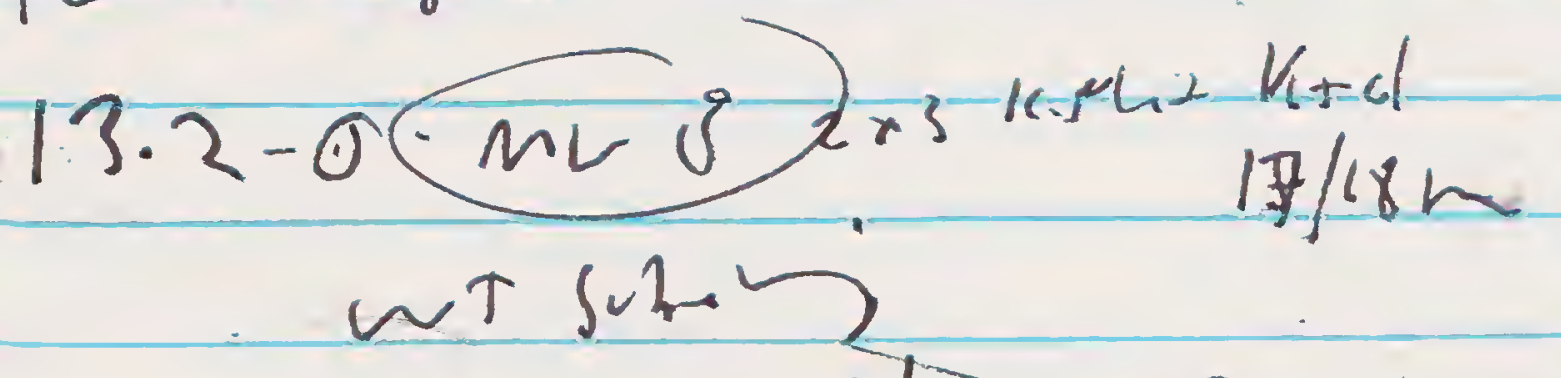

Rel. our red $\mathrm{mm} J \mathrm{i} / 15$.

Burabiil 
14.2 Rocts..4 11.2h

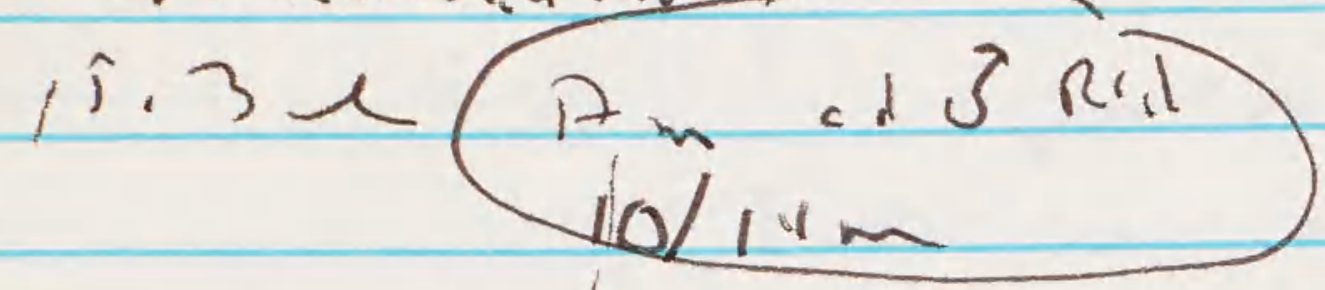

15,3 6.(we,bles!

[Hooded]

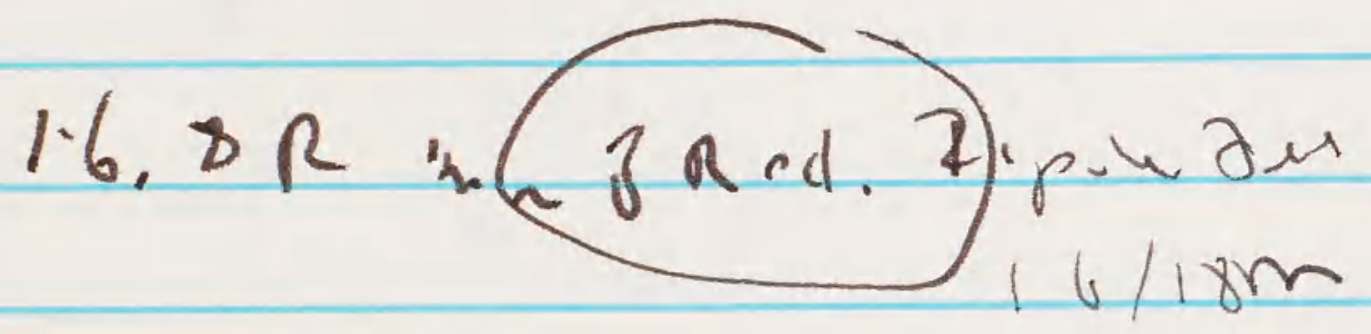

पT S.h.

I. B L oudrearer

$$
\frac{10.1}{12.8, R m \omega}
$$

B, B fly सूर

$$
\begin{aligned}
& \text { Baral) } \\
& \text { 14.y a L Ev Ld Jirsiry } \\
& \beta)^{b}=4
\end{aligned}
$$$$
M v=9
$$$$
n=9
$$$$
4 \pi^{0}=1,3
$$

$\beta^{+\omega}=1$

$$
\omega^{+w^{2}}=1
$$




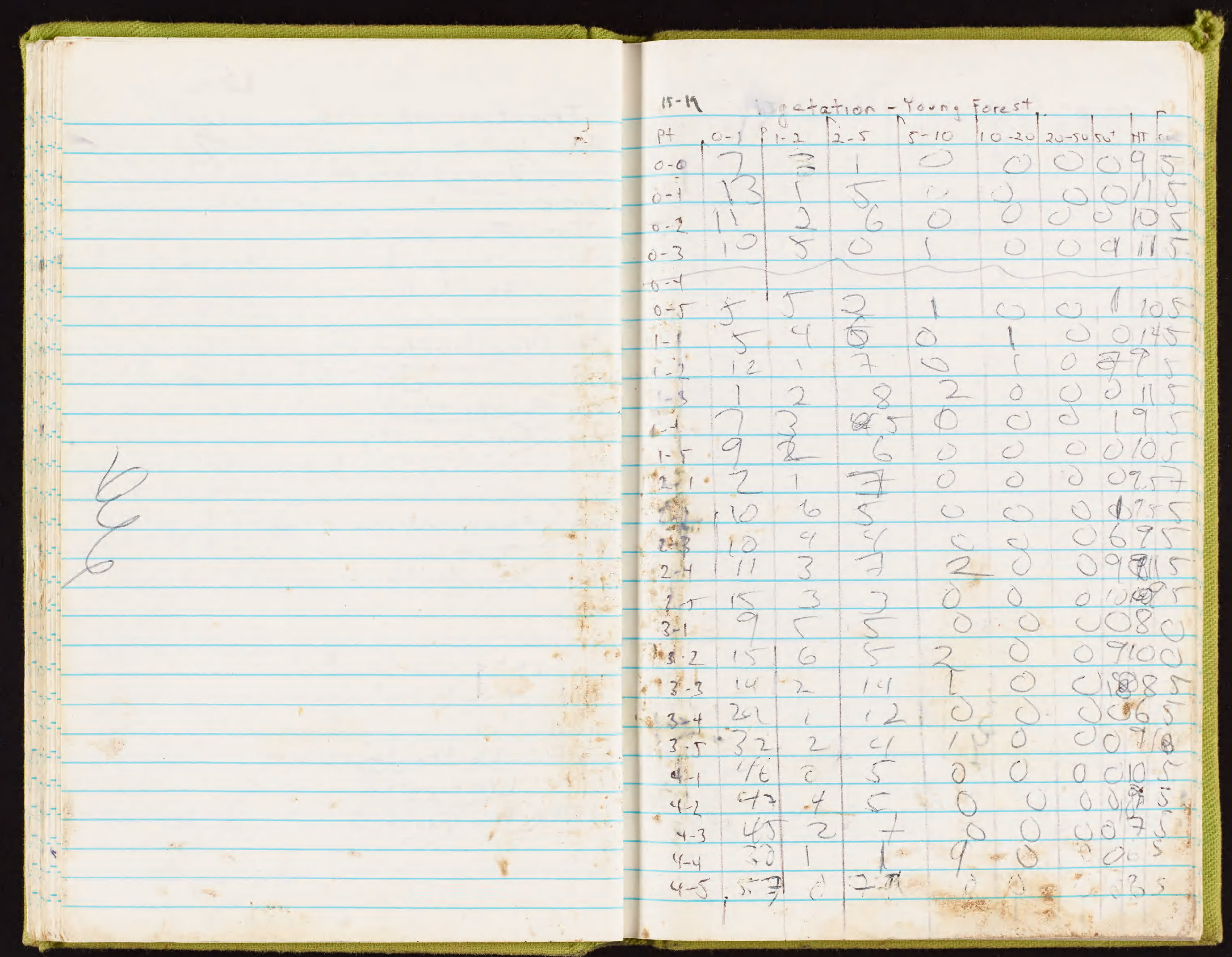




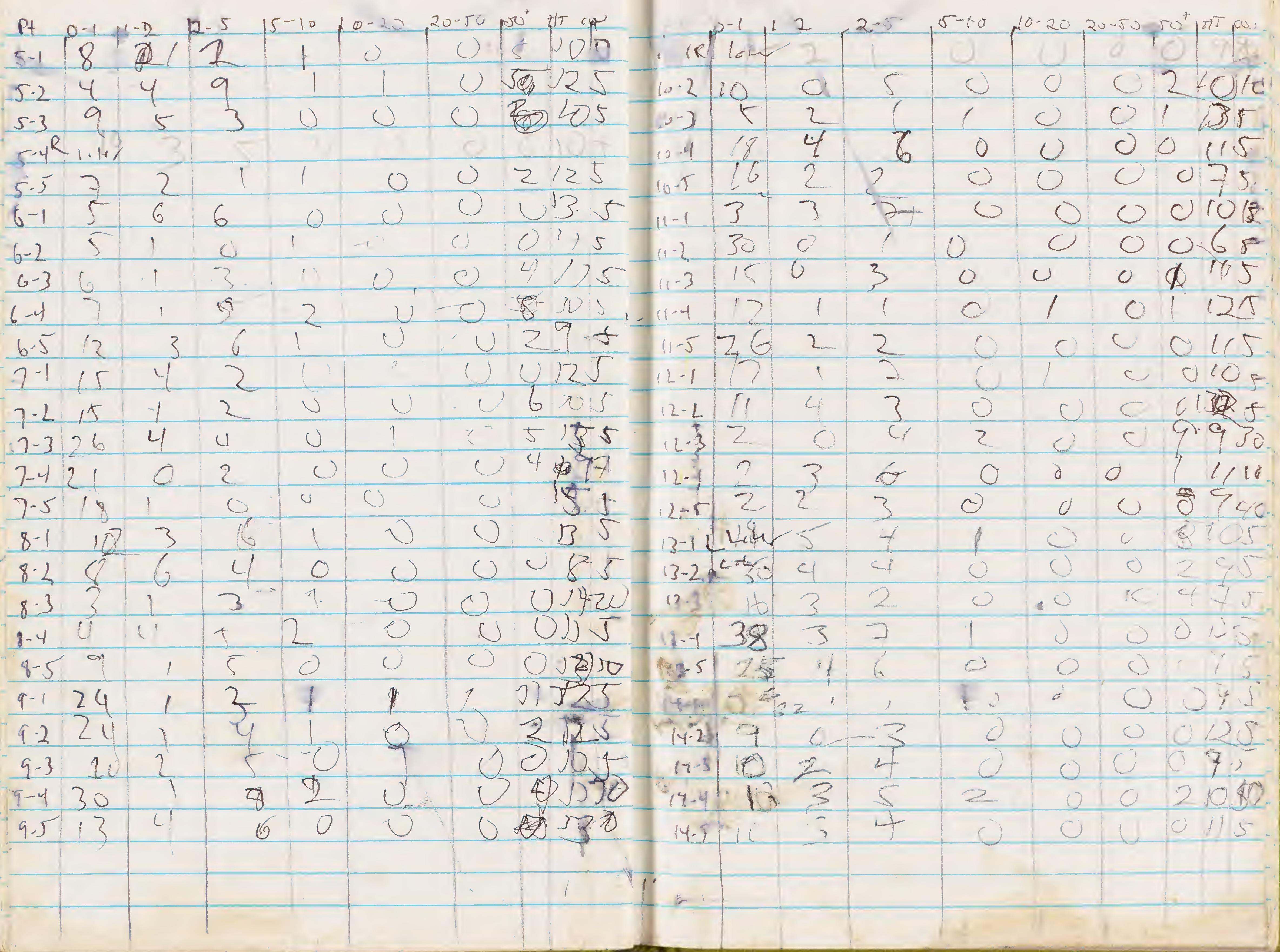




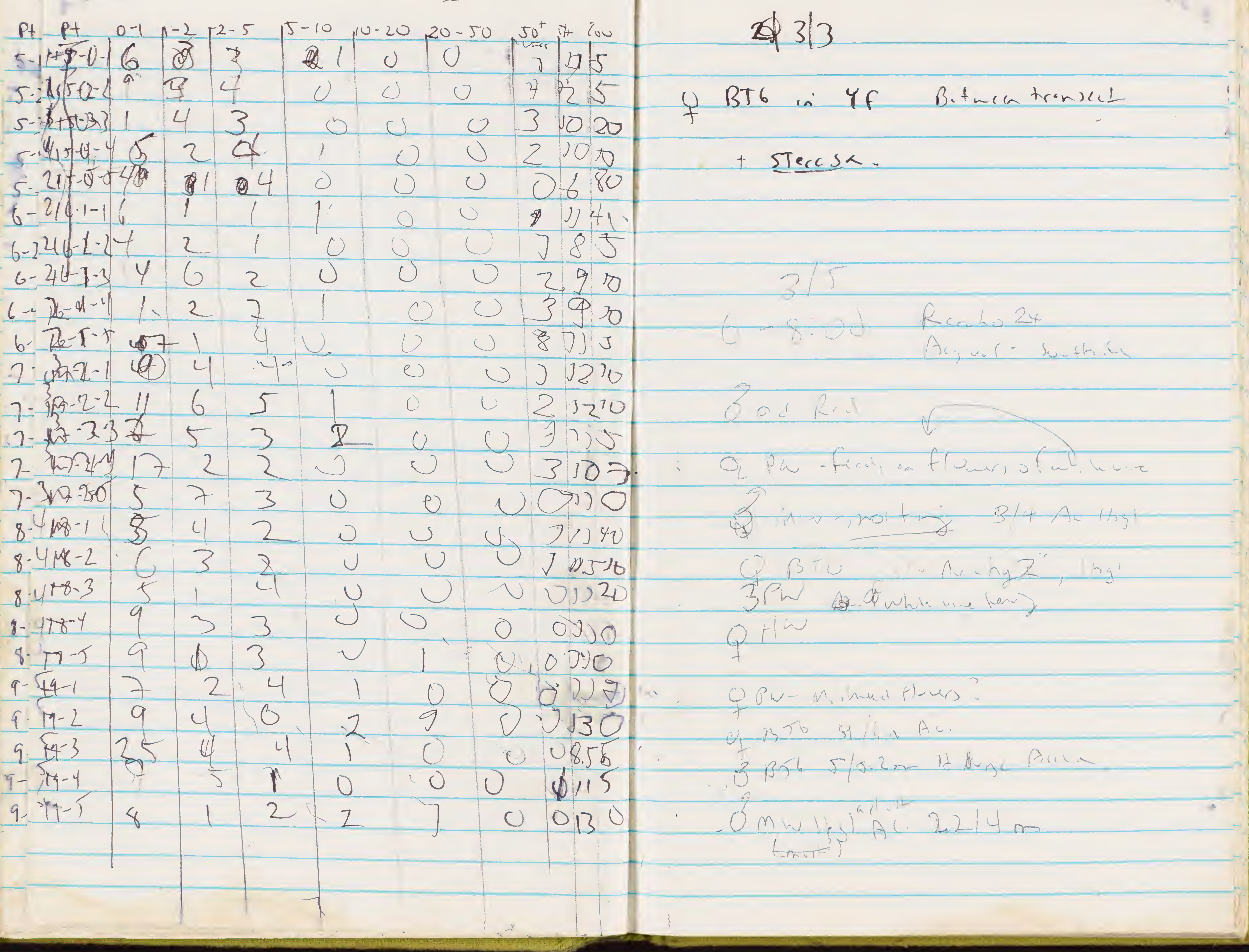




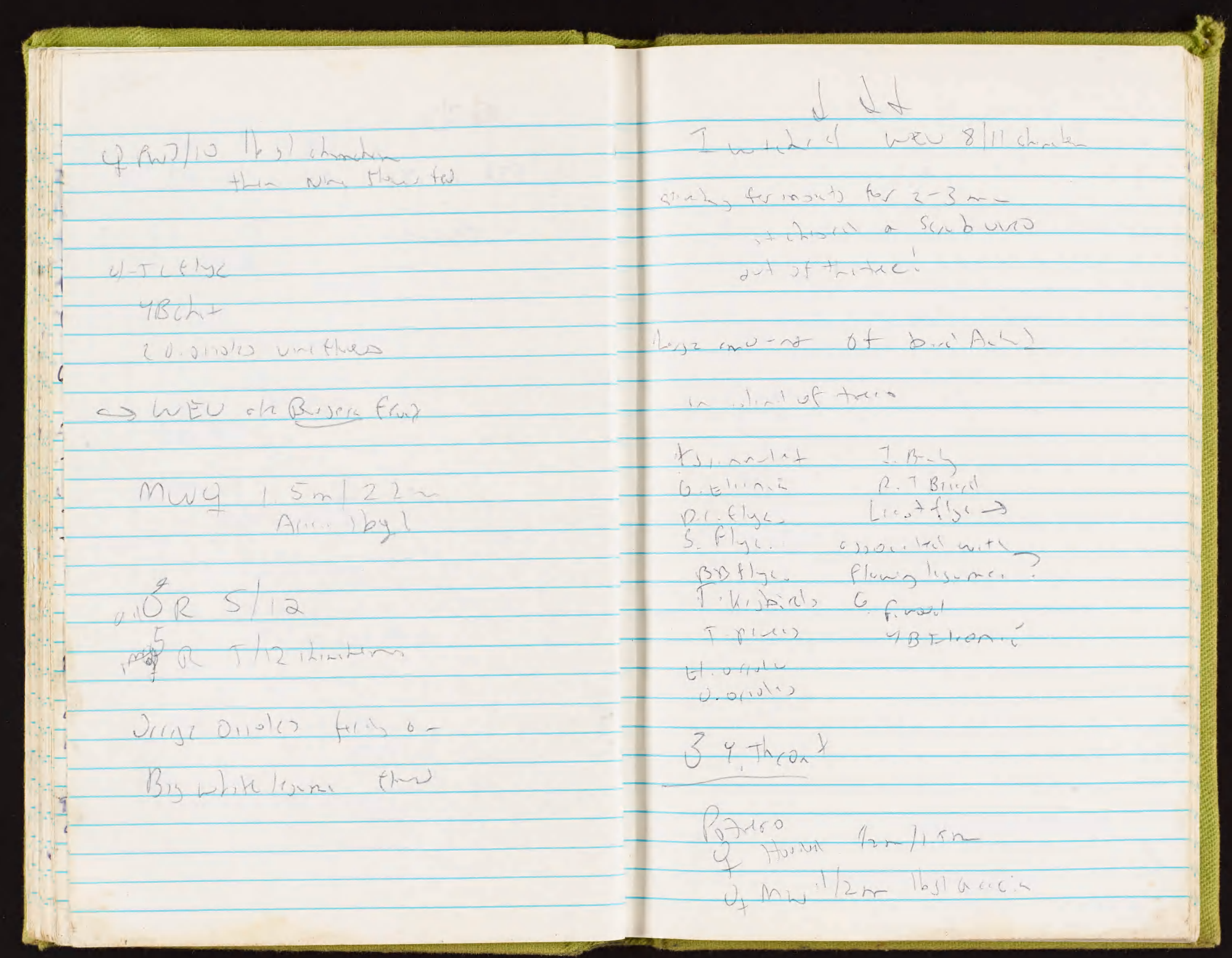


Ork, bouk in gury bexk

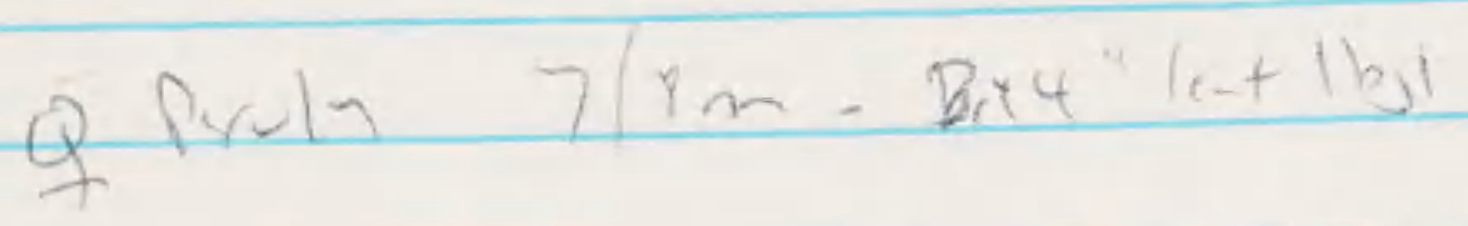

of $B 5-7(8 x A+2 ; 16 j$

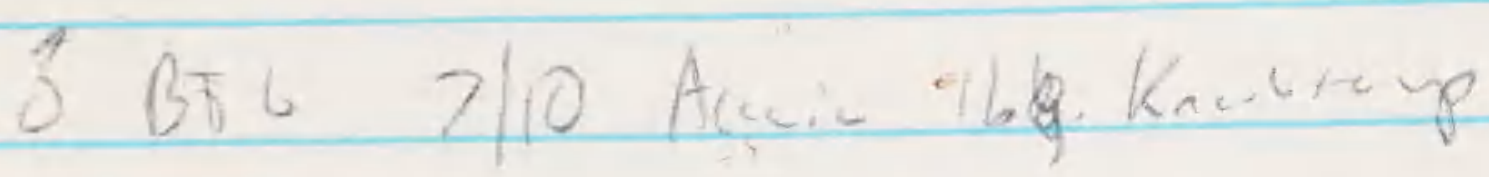

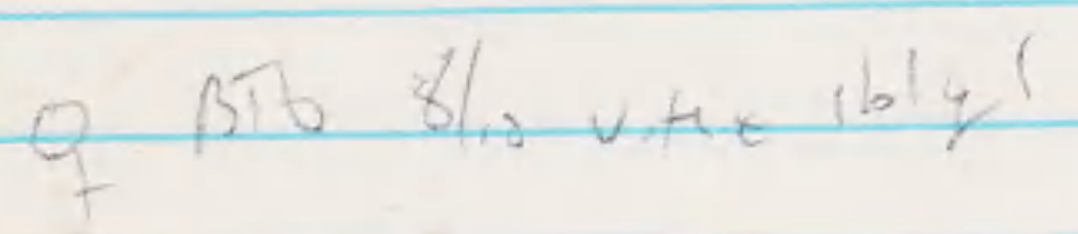

had 2 WES.

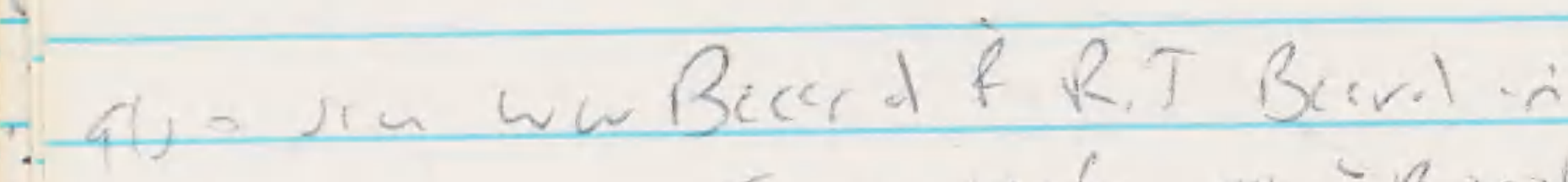

Seme Muf wTL T.B onde + T.hos
$3 / 6$ unjuzzal Angul dear-PC

50-(19dicul

19.4 F.B hime is

18.5 Ro surrosing

10.2 R2S. WNO

7 a s vero?

16.4 , s $\beta$ wred

16 ? Bant, 2ukchil

15.? 6.5 inuripede

15.6 e2 20 NW moli $3.517 m$ aw"

15.2 wev swy

$15 x+B+c_{0}$

$14.82 \sin$

$14.5 \sqrt{43} 0.2^{n}=6,6$

B.8R HW infinstit?

$13.2 \beta . B+\cos (2)$

2.4 s.uiru

$20.2 f+j x$

12. J S. vाico

11.? R. T Eow'

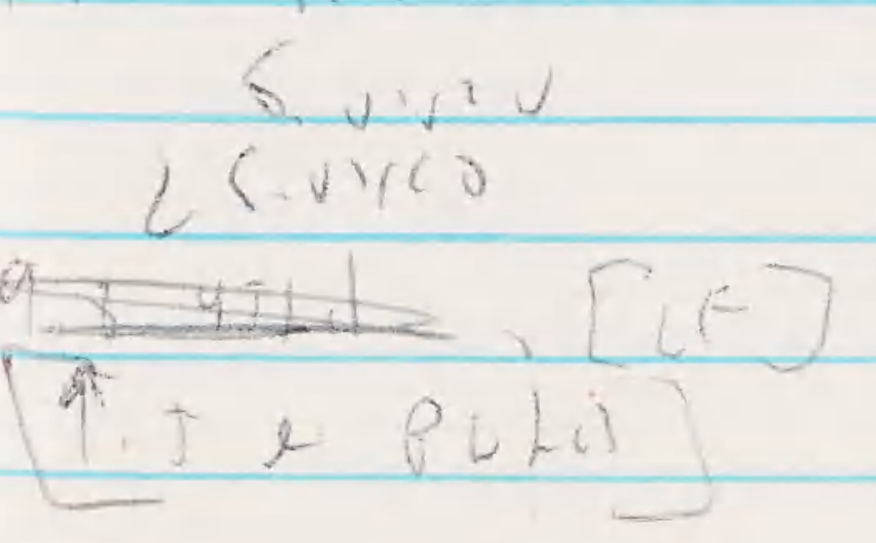




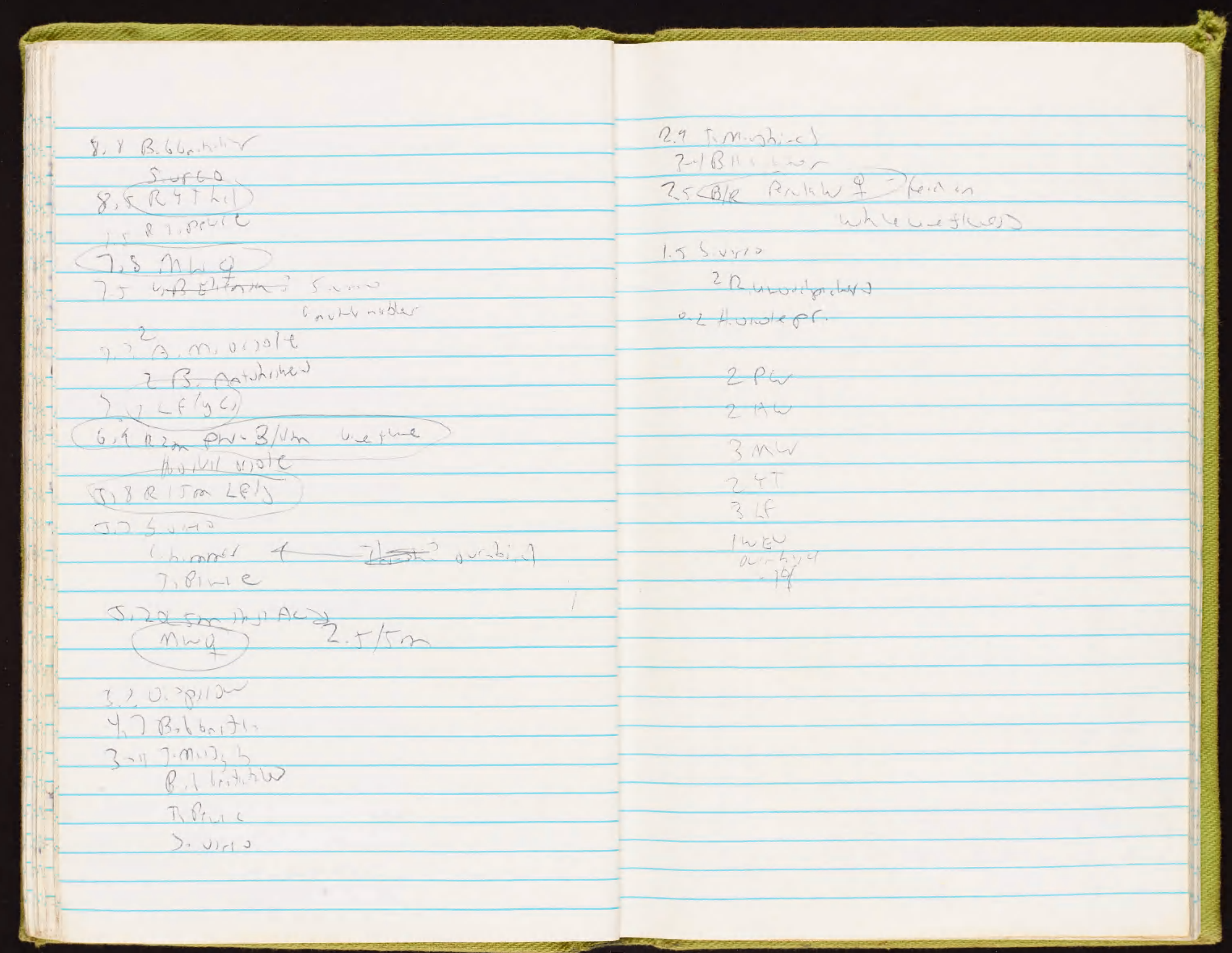




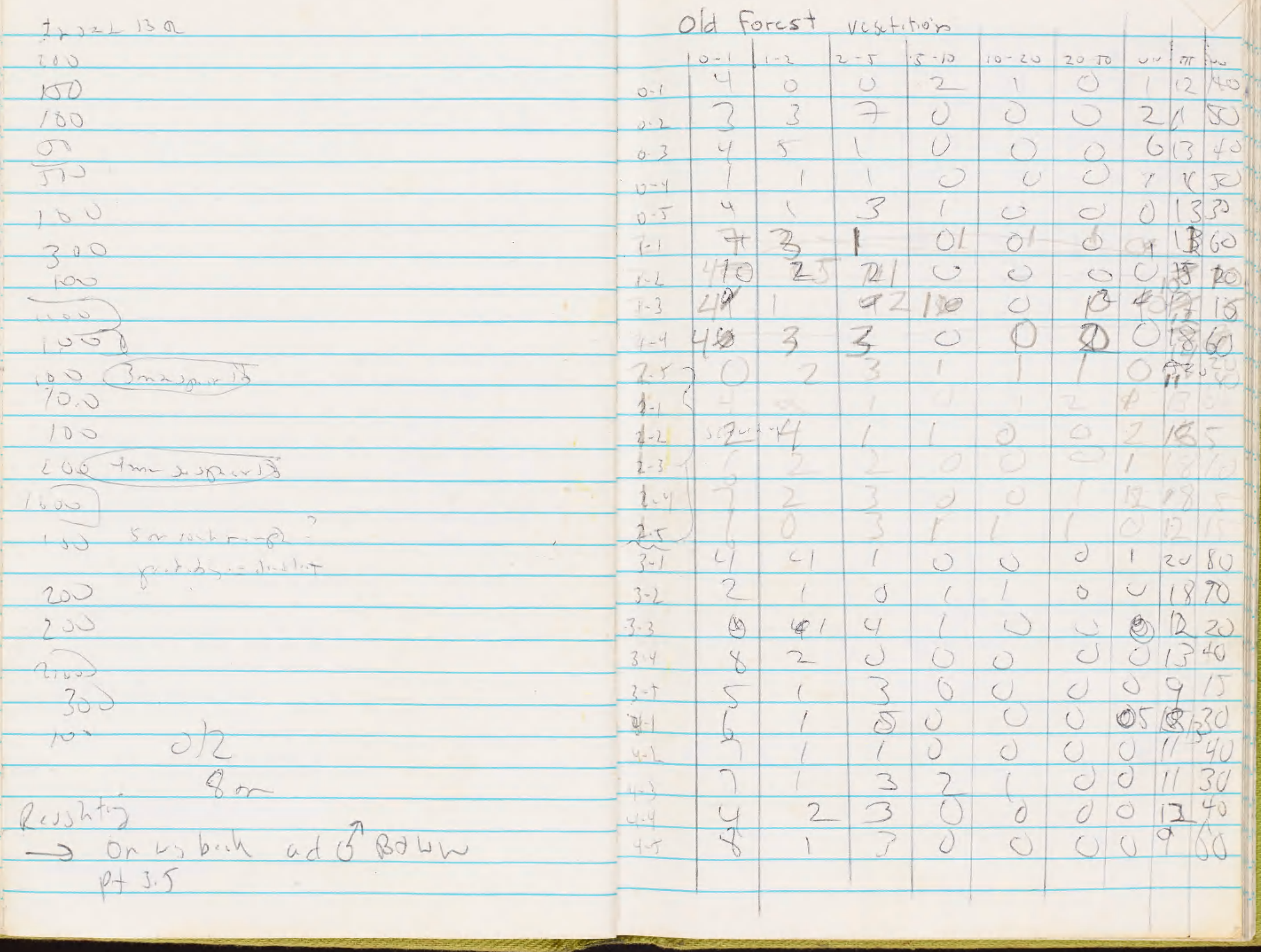




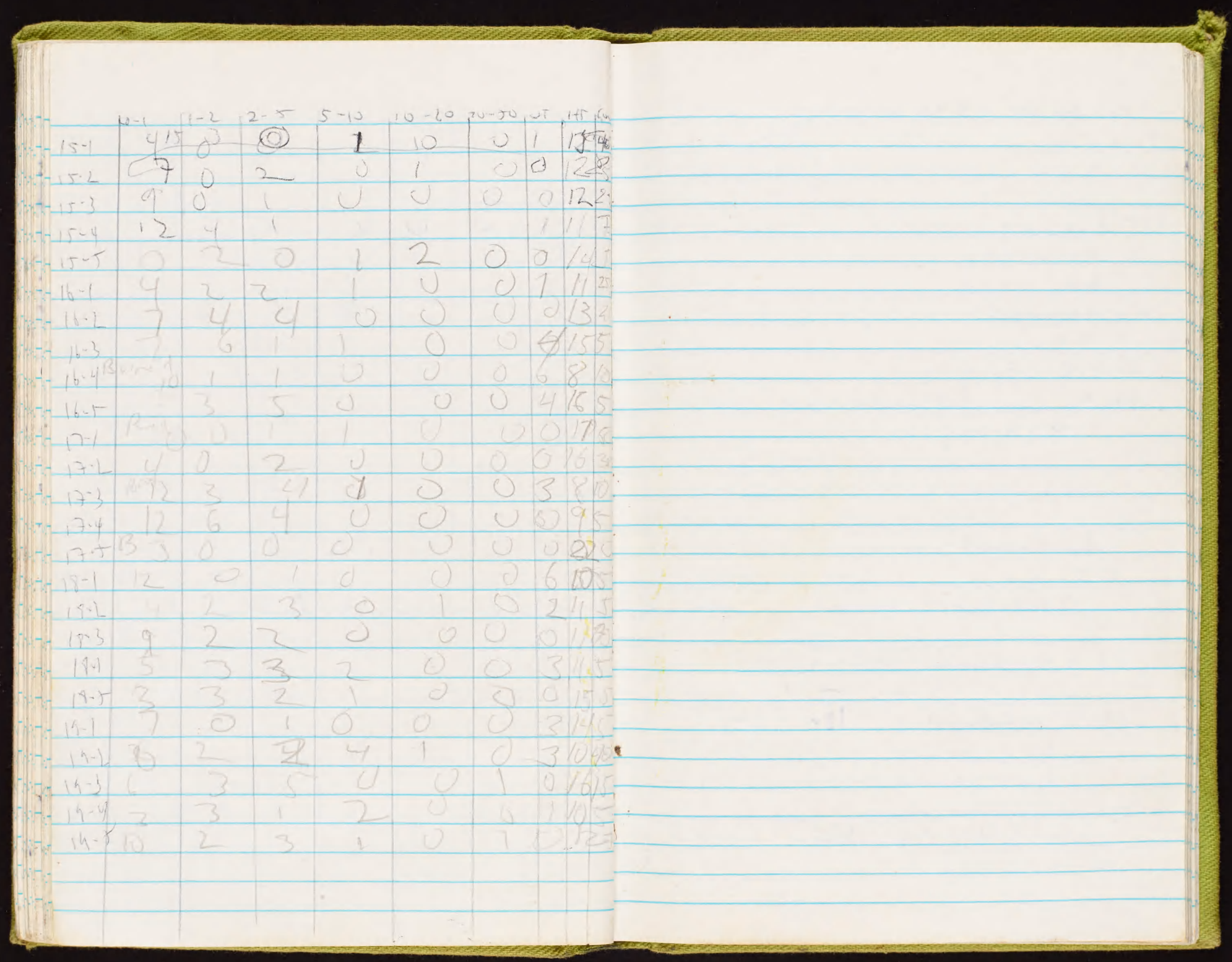




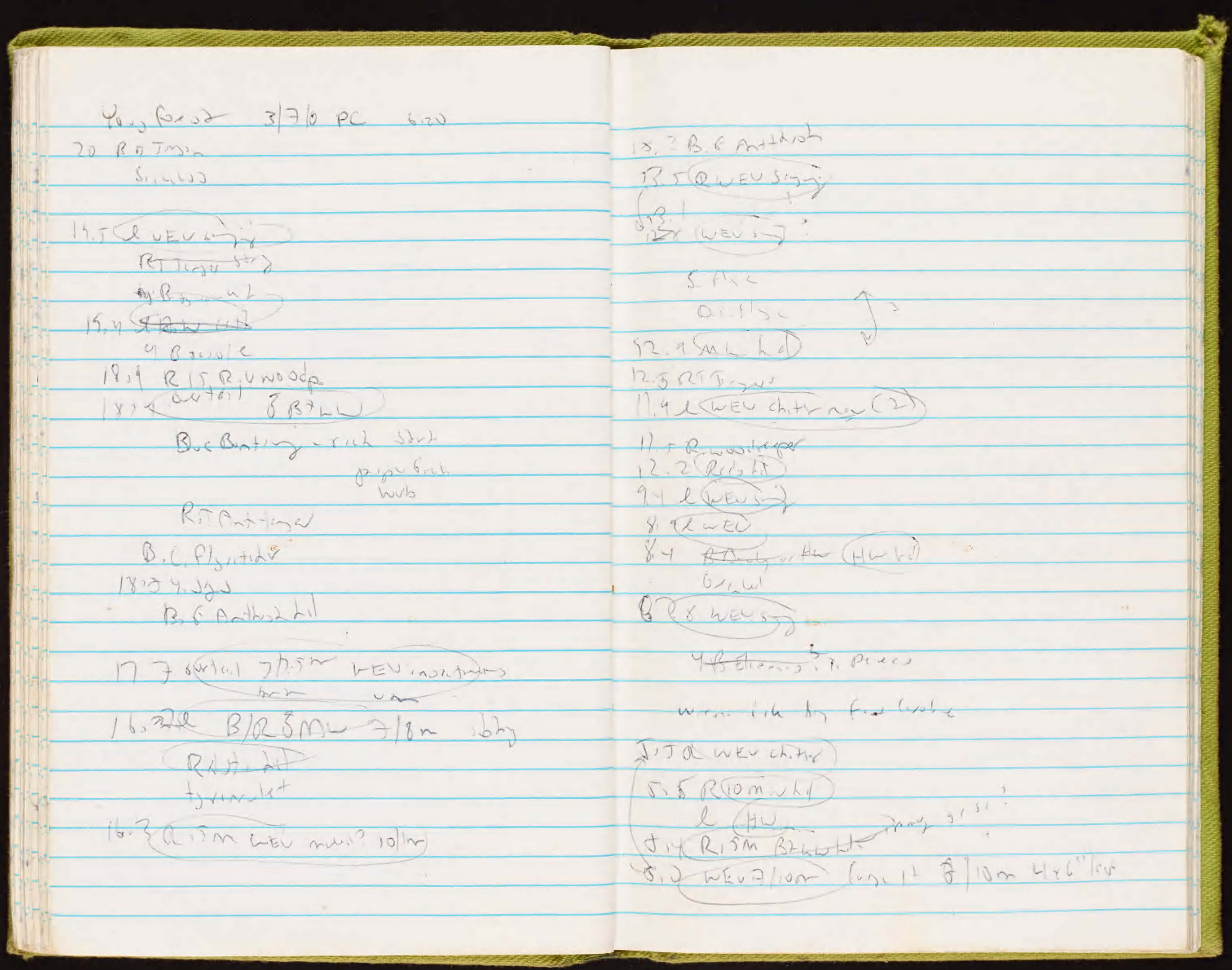




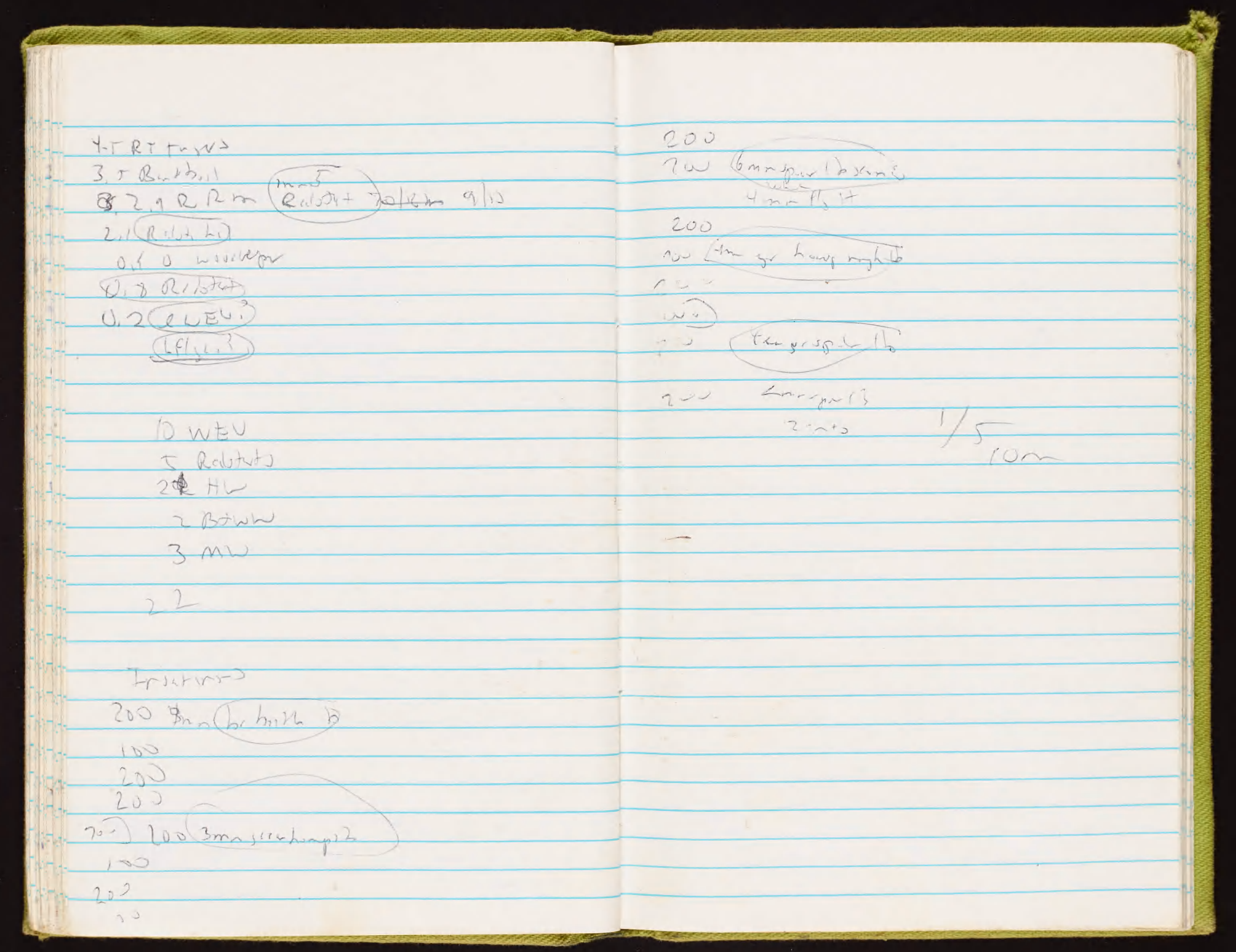




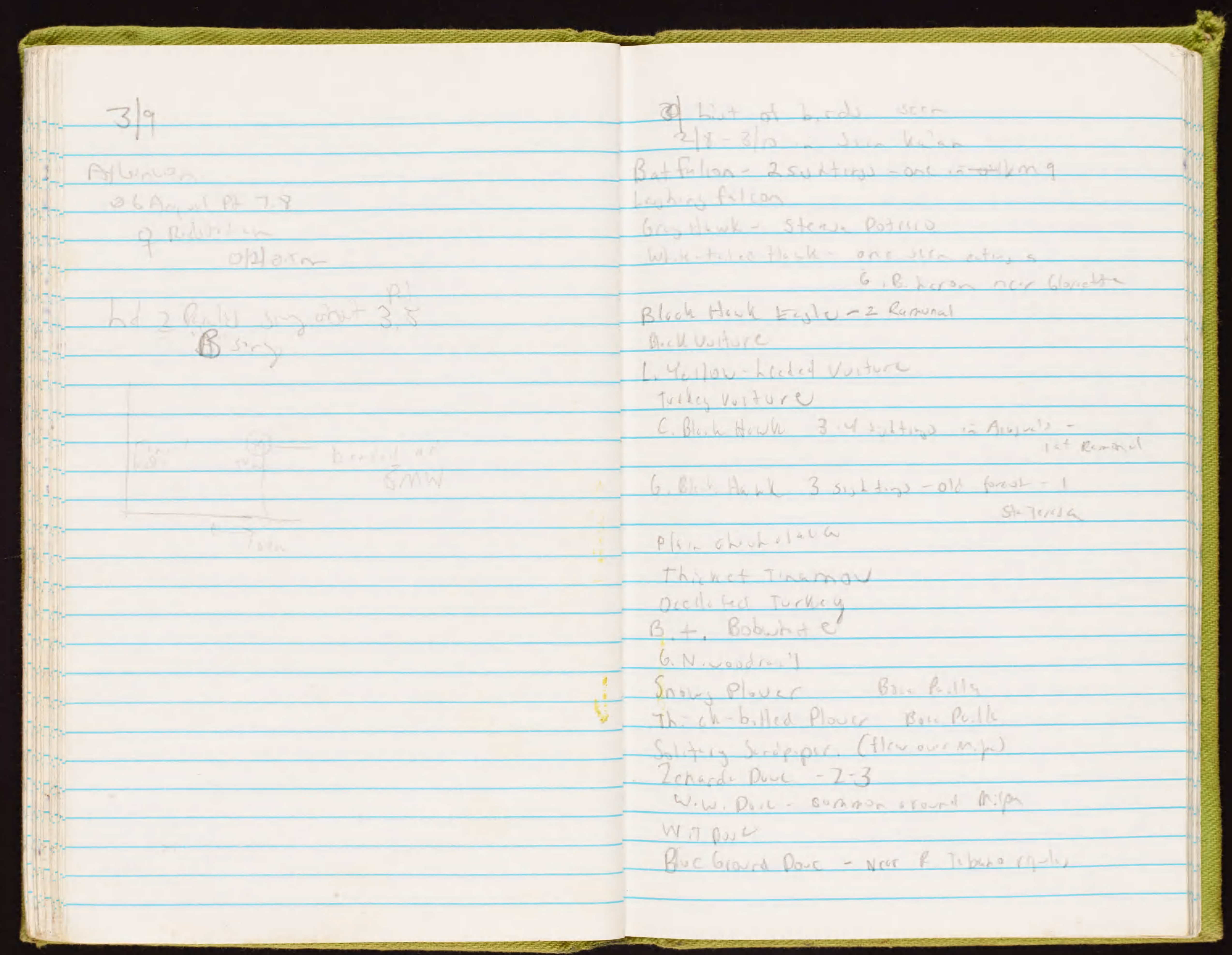




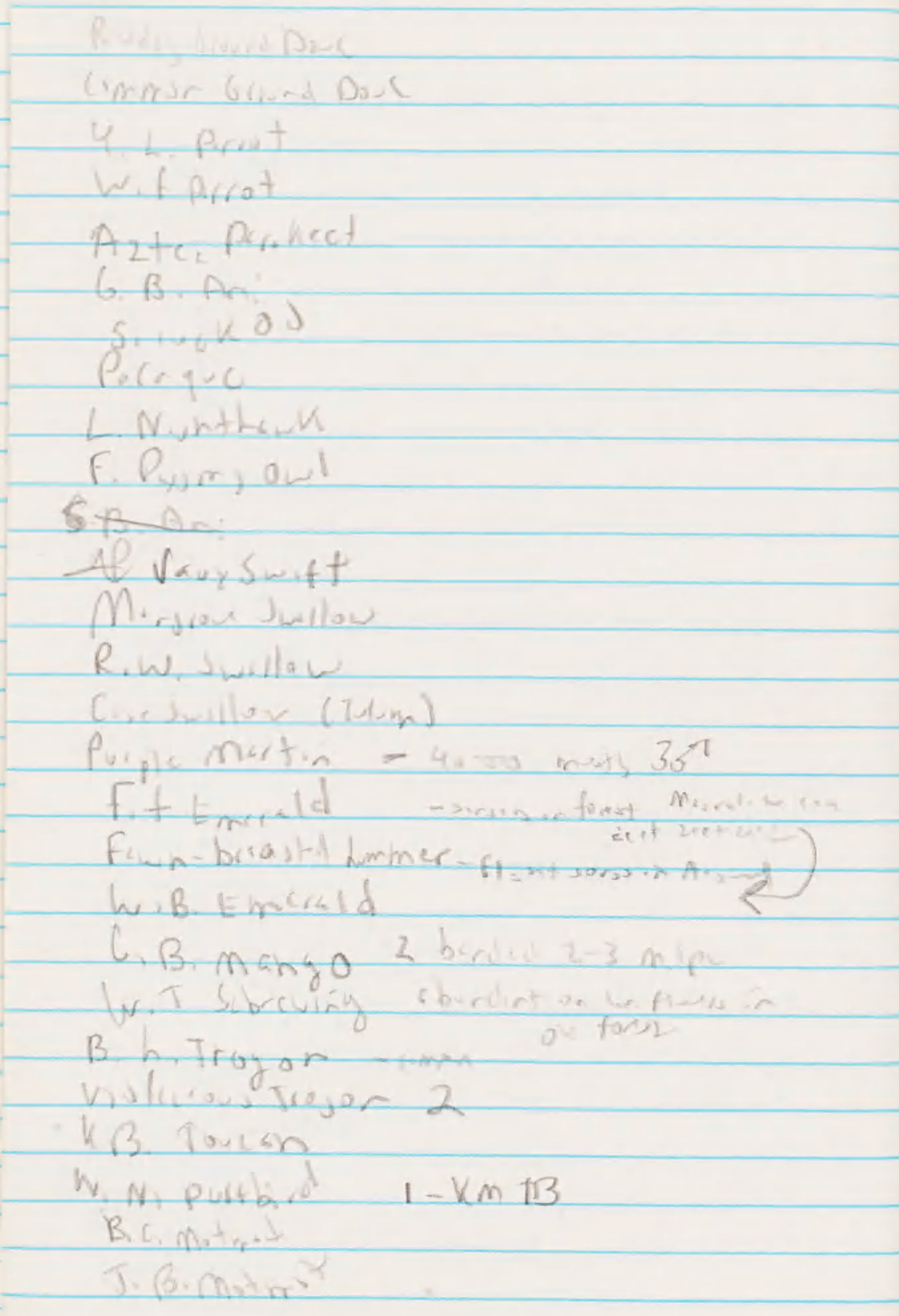

L.WAG b. whed whodpener

Linforth a wapes b

Guldeo-aliuchavipcaler

Rod-uentodwoud pak or

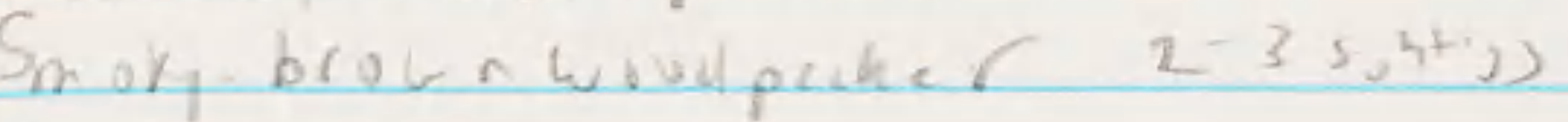

Pate bill oul wasedperher

Riddjwitalieper

trwoy wirged wood greper

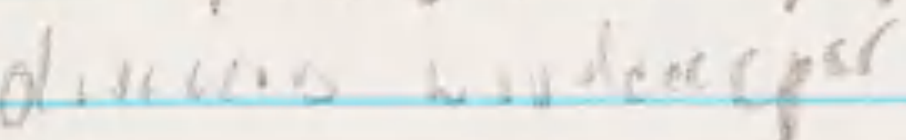

Ins bilted wirdecepor

R. B. puactio.

Plis a Y chap $25,2+100$ ol 8 fien

Birced Anturno

B. E A thish

$R, C$ mantion

B. R, Aflla

G) Becurd

RT Bcard

Mueded Thion

Joginil l g boul

Sound flutater

Buct b.llch fywlibr

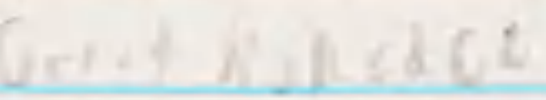

C.iffintifs

B.e figntihe

y.uth $(5,0) 6$

D.c. Hawh 
Trop:inl Prwo

Leis t flutedel

S. R. flyutiber

Nattein Rupl Hy, Lh

L. T. J p... bull

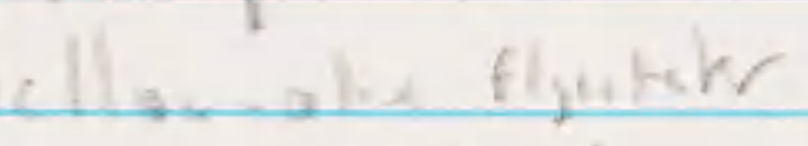

Eyares $(1 .+b .1)$

N. B $(n+b .1)$

4. B. Ellemia

Gormin Elcenia

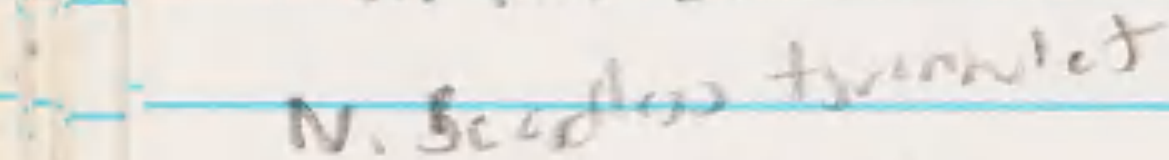

Q. B. HJotaber

Brown Jey

Green dag

Carblina wer

Sest brusted wron

Htors wirer

W. Bullard when

The pial Mockubied

Hose Thesh

Blue-gosy Gritutitid

Trapinal Go.tutho.?

LB Gotwre -

R. B peper shoikc
We anco

Morgate $a-r \in d$

$4 \pi, j \cdot r(2$

Ysestion virea - fow

Phechnesecer

Lesor Gesplet

Thentrowace besaley - fen

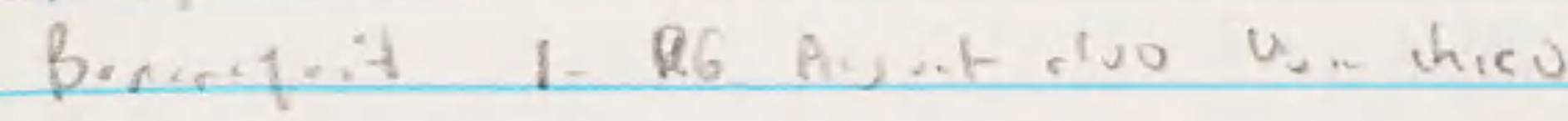

Blukerd wh te

\$2. Blat-wirje\& scurel

we wabler

NiPauk wardel

Yellow

M. nolia

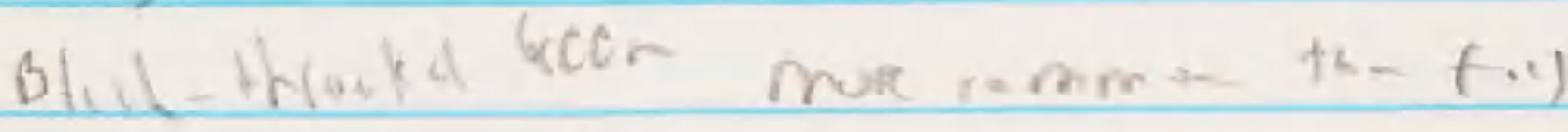

thellathonad

Patm

aubird

M wulert wish

Meatuals weiblel

C. Yuluthont

Ge vull-wtriout birgiry

4, hichit fow'

$6+$ chat

Hooded lables

Am eidutert 


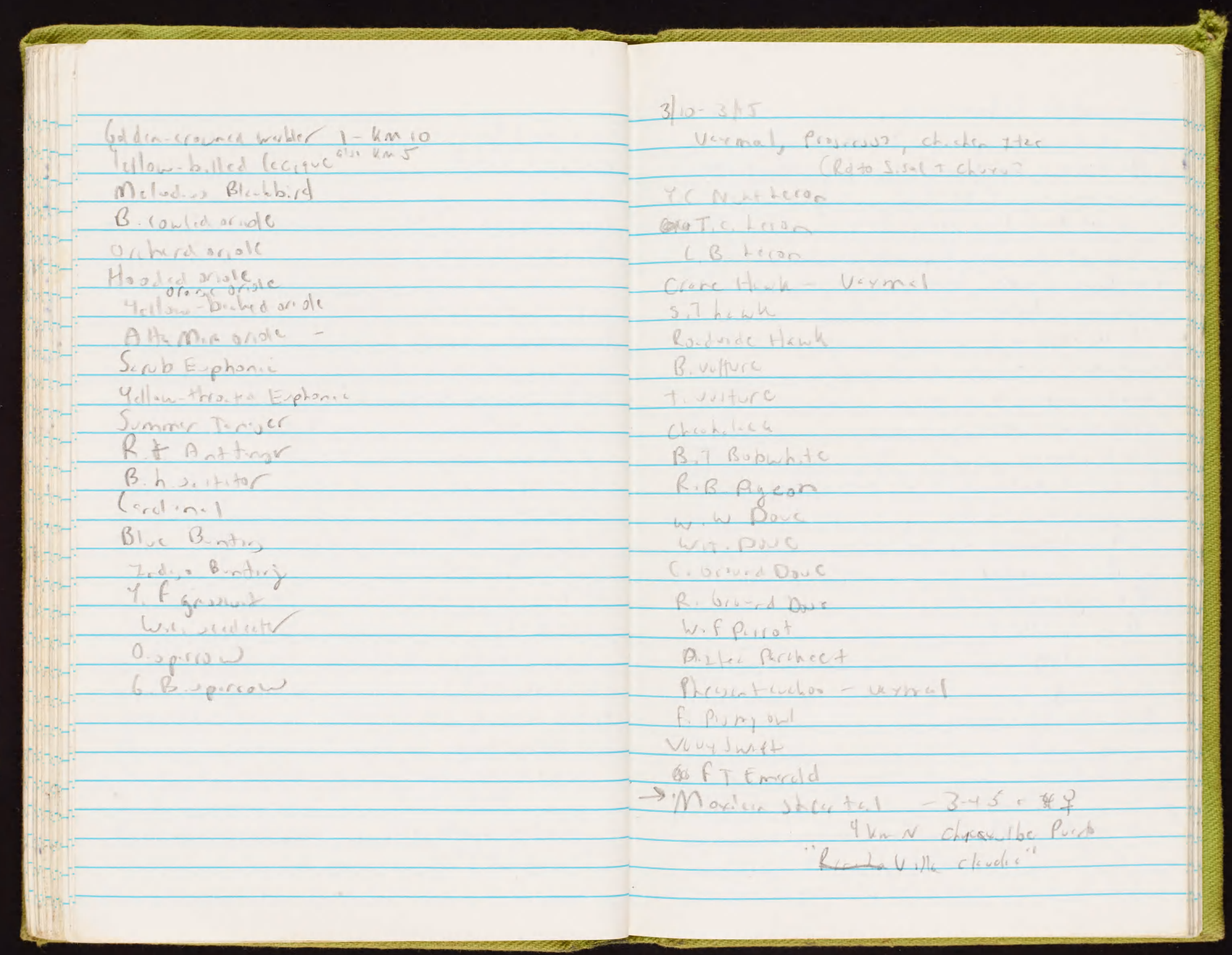




\section{$\$$ L.fer!}

checued a t coutt Surb or a

Ho Sor 1 - No flume - so

heyt moing $5.30-6 ; 0)$ che ext

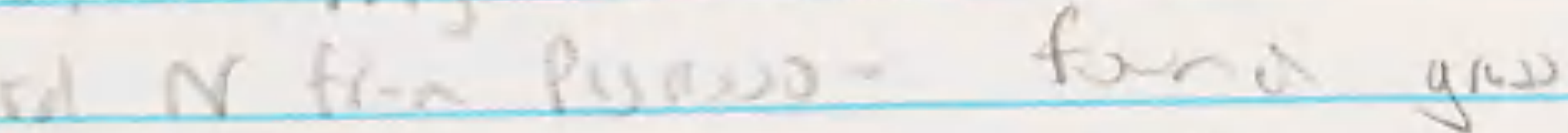

a r it puth ree seroph is boon

t dewr (6.00) humves beisme

(ative in (ib) $-2-33$ is

doji ploximty piunies t ticy

-in $q$ - thest enure puple the

Dowh - white abue whas \&ulor

thout wis griking

bit 7 and 30

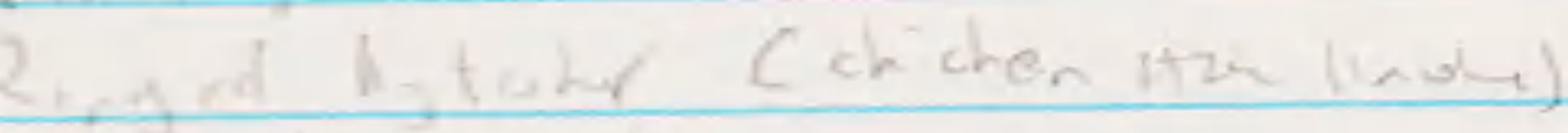

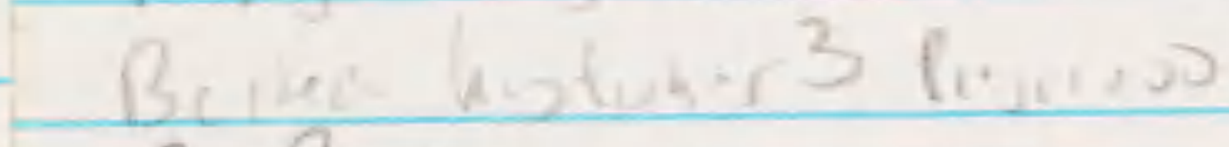

B Matmot C.I.

- B. butipivel

Q. B tipule, veymal

- B.C. H.w.

- Sow ity

B. B flg:

b. Ainkiles

Thy hibid

$1+p=1$ PrwLC

Virm.ll... flye thexulab N. Benalles binnalet

Cous dullow - nosting if Uimal
Grect do

$\{B=4-(i+\ln ) i y$ Brown J.y

Cralow were

1uretanwien - ehelem, che $x-1-b \rightarrow$

Hit an the J-6 sor of 2-y wrent

B. 6. Grotitith

trapic( M. t ybirel

Sarb

huE virso

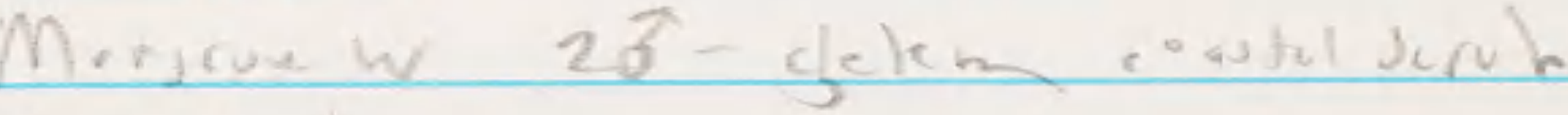

Ur Y,ilutares

His

$$
M+6
$$

uerm -1

An Rex ad है

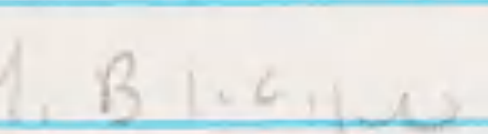

Ava mic orialc

Ooride outh

Bogale

S. Kerowih

B. B liwation

B) $B=-3$

Binua B-an/ ( 6.1$)$ 


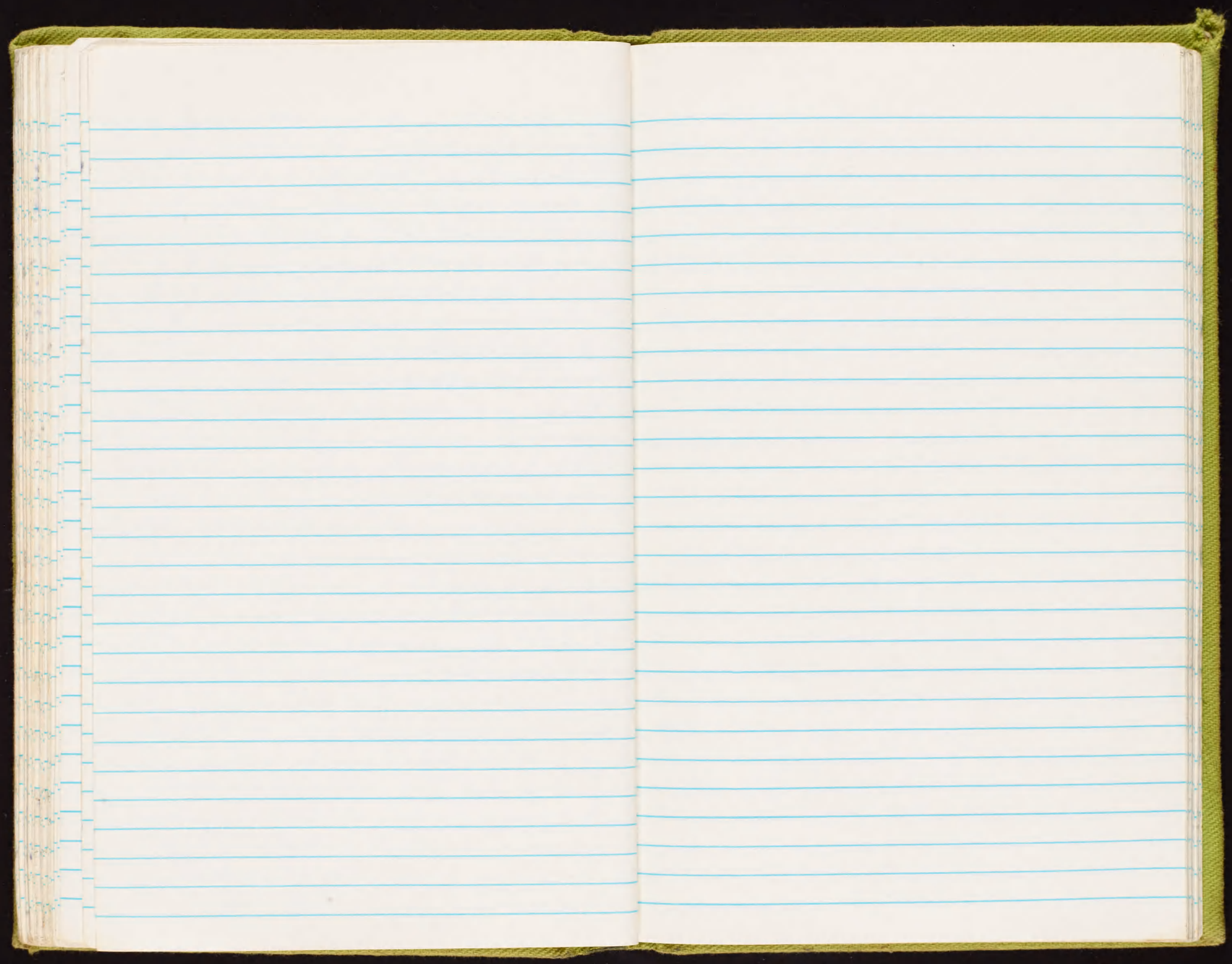




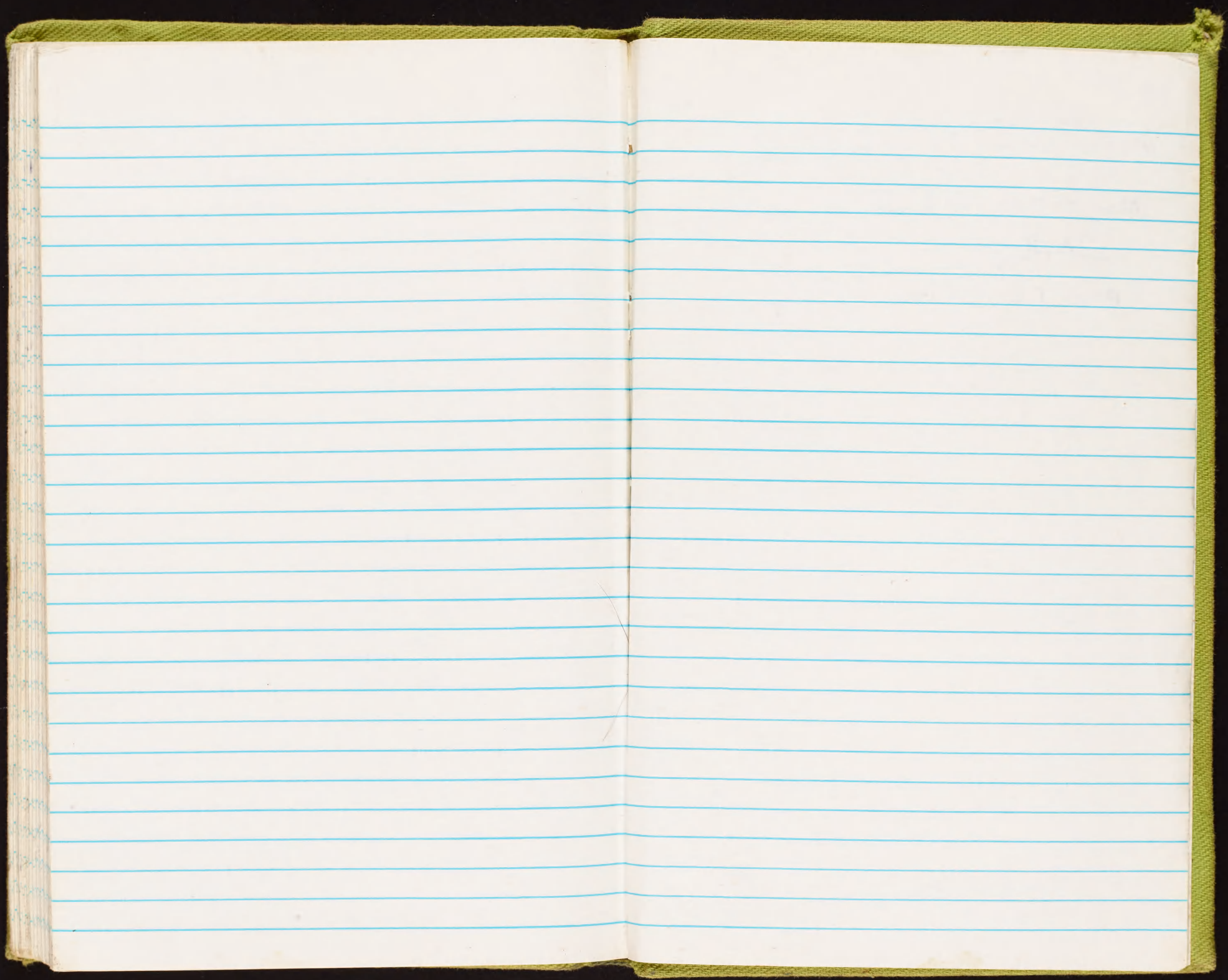




$$
\begin{aligned}
& \begin{array}{l}
\text { R.T ins } \\
\text { Musiut whow }
\end{array} \\
& \text { Chew whitw inewith hi wh } \\
& -1-1-1 \\
& \text { puph.f.ich butsiou }
\end{aligned}
$$




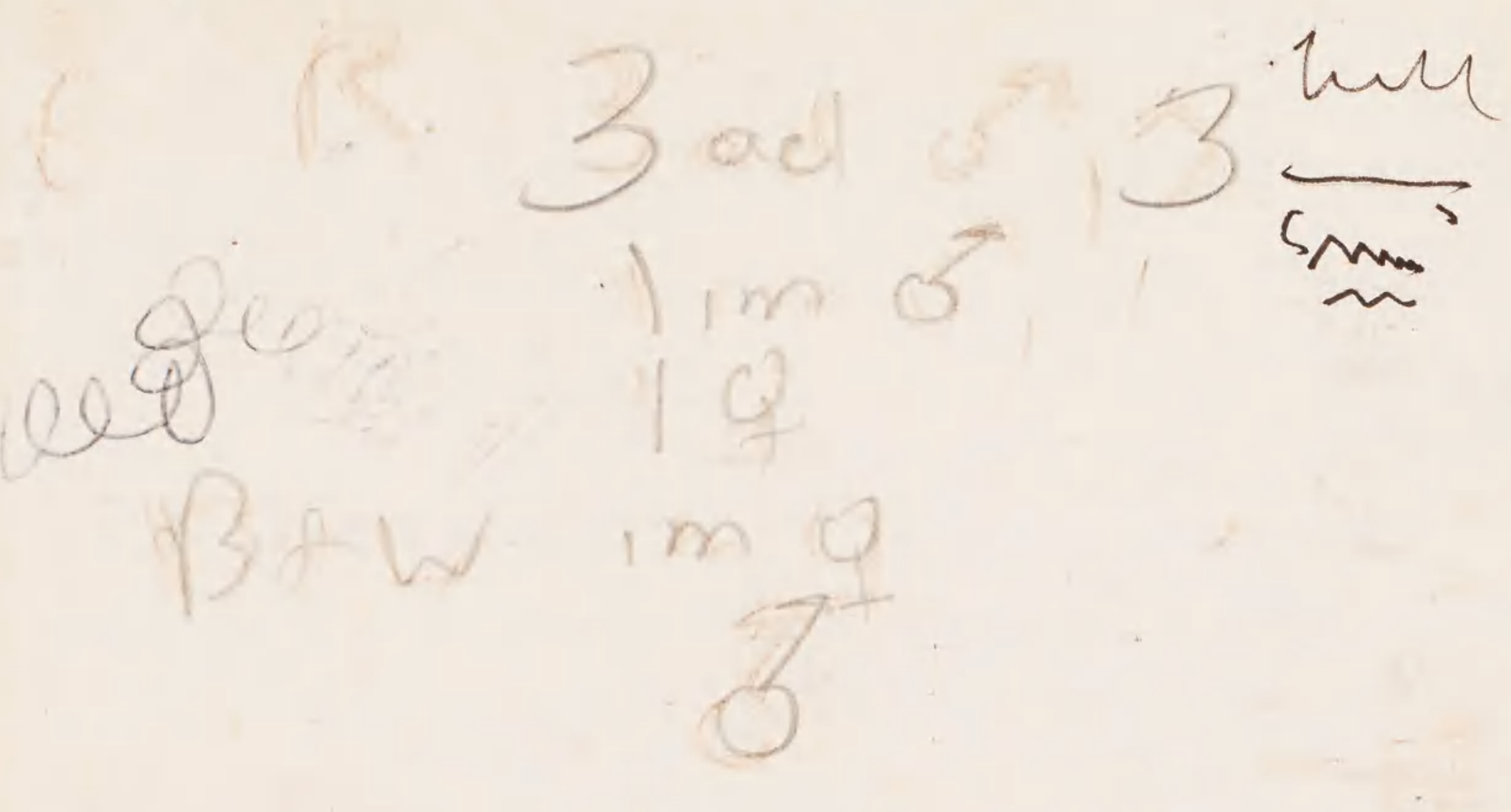

mw $2 q_{0}, 8$

$B+620^{\circ}$

Hit $x=3$

P.W.

v 3 .

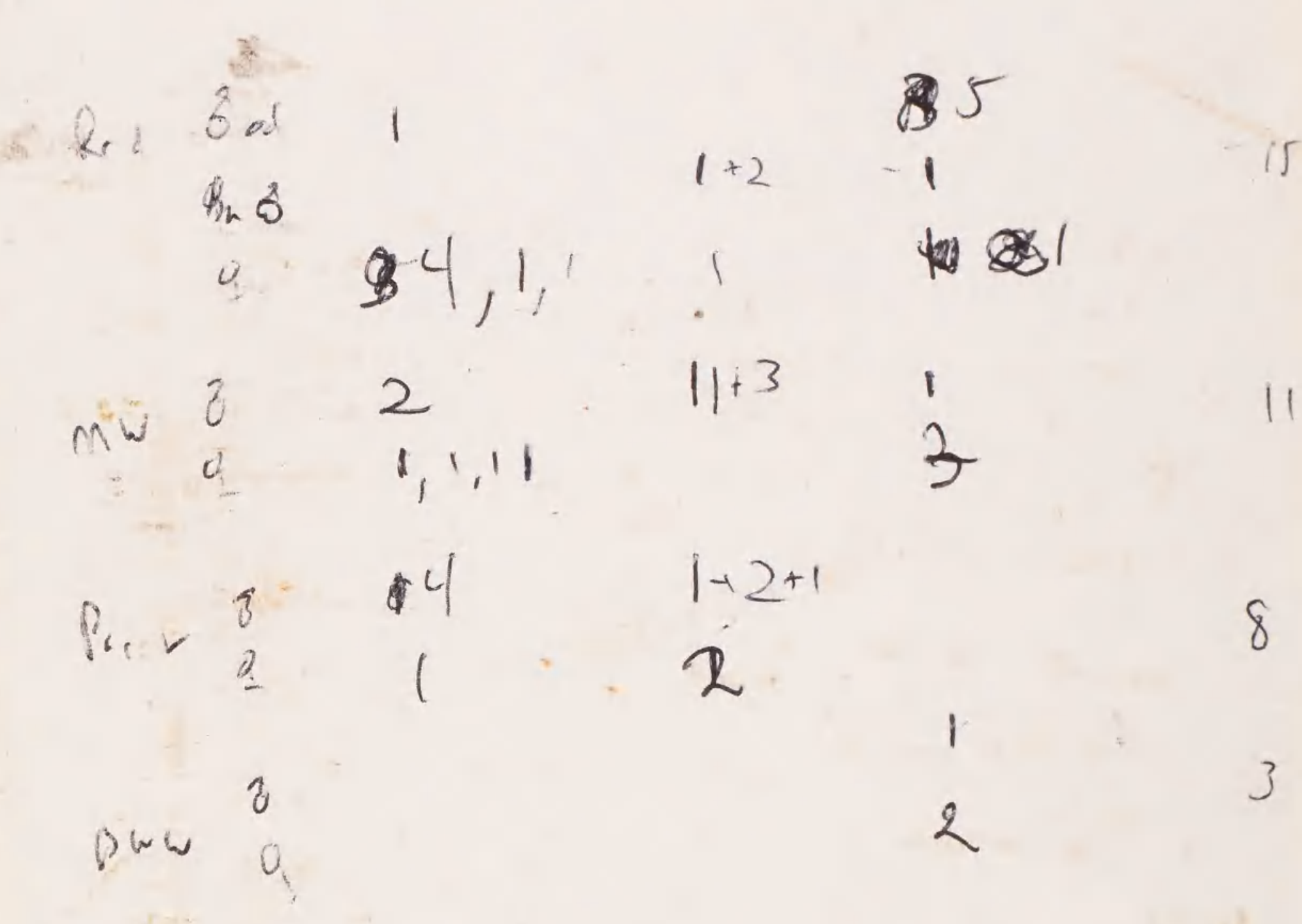

it $3 \cdots 1$

2

B. 36

3

16

18

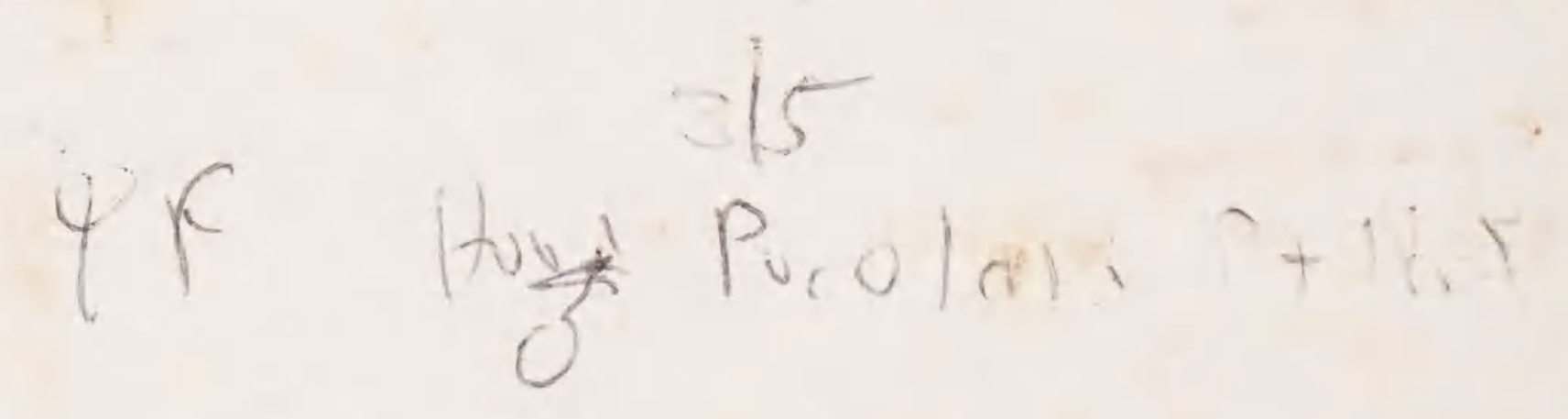


